\title{
Synthesis of (-)-Piperitylmagnolol featuring ortho-Selective Deiodination and Pd-Catalyzed Allylation
}

Atsushi Ikoma, Narihito Ogawa, Daiki Kondo, Hiroki Kawada, and Yuichi Kobayashi*

Department of Bioengineering, Tokyo Institute of Technology

Box B-52, Nagatsuta-cho 4259, Midori-ku, Yokohama 226-8501, Japan

Summary of $o$-Regioselective Deiodination of $o-, p$-Diiodophenols $\quad \mathrm{S} 2$

$\begin{array}{ll}\text { Table S1 S3 } & \text { S1 }\end{array}$

Experimental $\quad$ S4

General $\quad$ S4

A Preliminary 1,4-Addition (Scheme 2, eq 4 in the text) S4

Synthesis of (-)-Piperitylmagnolol S5

Synthesis of $o-, p$-Diiodophenols $\quad \mathrm{S} 11$

$o$-Selective Deiodination of $o-, p$-Diiodophenols $\quad \mathrm{S} 13$

$\begin{array}{lr}\text { References } & \text { S15 }\end{array}$

Determination of Enantiomeric Ratios $\quad$ S16

${ }^{1} \mathrm{H}$ and ${ }^{13} \mathrm{C}$ NMR Spectra to Establish Identity and Purity S18 


\section{Summary of $o$-Regioselective Deiodination of $o$-,p-Diiodophenols}

(Table 1, entries 1-5 in the text: Table S1, entries S1, S7, S2, S17, S18 in this supporting info)

The reaction was first examined with $\mathrm{LiAlH}_{4}$ in $\mathrm{Et}_{2} \mathrm{O}$ at $0{ }^{\circ} \mathrm{C}$ and quenched after 30 min. Although the reaction was not completed, a mixture of the desired products $\mathbf{2 1}$, regioisomer $\mathbf{2 2}$, the over reduction product 23, and the starting iodide $\mathbf{2 0}$ was obtained (Table S1, entry S1). A metal hydride examined next was $\mathrm{NaAlH}_{2}\left(\mathrm{OC}_{2} \mathrm{H}_{4} \mathrm{OMe}\right)_{2}$ known as Red-Al, with which the deiodination in $\mathrm{Et}_{2} \mathrm{O}$ at $0{ }^{\circ} \mathrm{C}$ proceeded regioselectively and rapidly to afford 21 in $79 \%$ isolated yield (entry S2). On the other hand, no reaction took place with $\mathrm{BH}_{3}$ in $\mathrm{THF}$ and with $\mathrm{NaBH}_{4}$ in $\mathrm{Et}_{2} \mathrm{O}$ and in DMF (entries S3-S5), probably because of the weak nucleophilicity of the hydride on the borane. The $\mathrm{NaBH}_{4} / \mathrm{NiCl}_{2}$ complex, developed for debromination and dechlorination, ${ }^{\mathrm{S} 1}$ resulted in slow reaction and low selectivity (entry S6). No deiodination was observed with DIBAL $\left(i-\mathrm{Bu}_{2} \mathrm{AlH}\right)$ and with $\mathrm{K}$-Selectride $\left(\mathrm{KB}(\mathrm{H})(s-\mathrm{Bu})_{3}\right)$, recovering diiodide 20 cleanly (entries S7 and S8). In addition, metal-iodine exchange was examined with an expectation that chelation of the metal cation to the phenoxide oxygen might induce preferential attack to the iodine atom at the ortho position. However, the attempted deiodination with $n-, s$-, and $t$-BuLi gave a mixture of 21, 22, 23, and/or 20 (entries S9-S11). Deiodination did not take place with $i-\mathrm{PrMgCl} \cdot \mathrm{LiCl}($ entry $\mathrm{S} 12)$.

With the result obtained with Red-Al in $\mathrm{Et}_{2} \mathrm{O}$ (entry 2), other solvents were examined next. THF gave a similar result to that obtained in $\mathrm{Et}_{2} \mathrm{O}$ (entry S13), whereas DMF and DMA lowered the regioselectivity and reactivity of the deiodination (S14 and footnote $b$ ). Among non-polar solvents, toluene showed sufficient efficiency giving 2a in 87\% yield (entry S15), whereas the reaction in hexane proceeded slowly (entry 16). Deiodination in $\mathrm{CH}_{2} \mathrm{Cl}_{2}$ proceeded with high efficiency as well (entry S17). Among the efficient solvents used in entries $\mathrm{S} 2, \mathrm{~S} 13, \mathrm{~S} 15$, and $\mathrm{S} 17, \mathrm{CH}_{2} \mathrm{Cl}_{2}$ was selected for further investigation because of the somewhat better selectivity and yield than others. A longer period of time $(3 \mathrm{~h})$ applied to the deiodination in $\mathrm{CH}_{2} \mathrm{Cl}_{2}$ caused little over reduction (entry S18). Furthermore, the use of 2.5 equiv of Red-Al was sufficient for completion of the reaction (footnote $\mathrm{c}$ ). Thus, the reaction time of $0.5-3 \mathrm{~h}$ and the quantity of 2.5-3.4 equiv are recommended for the deiodination. The protocol used in entry S17 was applied successfully to other iodides shown in Scheme 4 in the text. 
Table S1. Preliminary Results

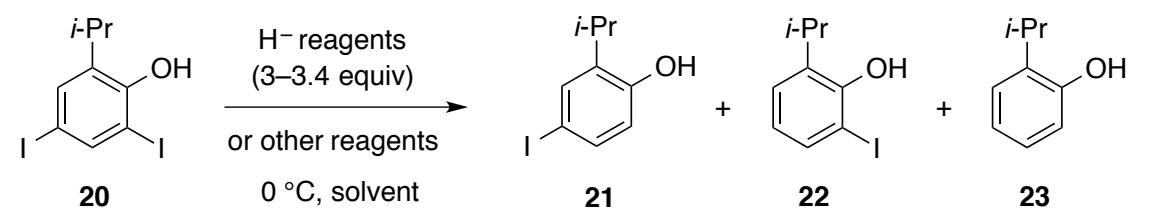

\begin{tabular}{|c|c|c|c|c|c|c|}
\hline entry & reagent (equiv) & solvent & $\begin{array}{l}\text { temp. } \\
\left({ }^{\circ} \mathrm{C}\right)\end{array}$ & $\begin{array}{l}\text { time } \\
\text { (h) }\end{array}$ & $21: 22: 23: 20^{a}$ & $\begin{array}{l}\text { yield } \\
(\%)\end{array}$ \\
\hline S1 & $\mathrm{LiAlH}_{4}(3)$ & $\mathrm{Et}_{2} \mathrm{O}$ & 0 & 0.5 & $30: 18: 8: 44$ & - \\
\hline S2 & Red-Al (3.4) & $\mathrm{Et}_{2} \mathrm{O}$ & 0 & 0.5 & $96: 0: 4: 0$ & 79 \\
\hline S3 & $\mathrm{BH}_{3} \cdot \mathrm{THF}(3)$ & $\mathrm{THF} / \mathrm{Et}_{2} \mathrm{O}(1: 3)$ & 0 & 0.5 & 0:0:0:100 & - \\
\hline S4 & $\mathrm{NaBH}_{4}(3)$ & $\mathrm{Et}_{2} \mathrm{O}$ & 0 & 0.5 & 0:0:0:100 & - \\
\hline S5 & $\mathrm{NaBH}_{4}(3)$ & $\mathrm{DMF}$ & 0 & 0.5 & 0:0:0:100 & - \\
\hline S6 & $\begin{array}{l}\mathrm{NiCl}_{2}(2.5), \\
\mathrm{NaBH}_{4}(6), \\
\mathrm{MeO}\left(\mathrm{CH}_{2}\right)_{2} \mathrm{OH}(12)\end{array}$ & THF & 60 & 3 & $28: 7: 9: 56$ & - \\
\hline S7 & DIBAL (3) & $\mathrm{Et}_{2} \mathrm{O}$ & 0 & 0.5 & $0: 0: 0: 100$ & - \\
\hline S8 & K-Selectride (3) & $\mathrm{THF} / \mathrm{Et}_{2} \mathrm{O}(1: 3)$ & 0 & 0.5 & 0:0:0:100 & - \\
\hline S9 & $n$-BuLi (3) & $\mathrm{Et}_{2} \mathrm{O}$ & 0 & 0.5 & $37: 16: 35: 12$ & - \\
\hline $\mathrm{S} 10$ & $s$-BuLi (3) & $\mathrm{Et}_{2} \mathrm{O}$ & 0 & 0.5 & $28: 22: 11: 39$ & - \\
\hline S11 & $t$-BuLi (3) & $\mathrm{Et}_{2} \mathrm{O}$ & 0 & 0.5 & $0: 0: 100: 0$ & - \\
\hline S12 & $i-\operatorname{PrMgCl} \cdot \mathrm{LiCl}(3)$ & $\mathrm{THF} / \mathrm{Et}_{2} \mathrm{O}(1: 2)$ & 0 & 0.5 & $0: 0: 0: 100$ & - \\
\hline $\mathrm{S} 13$ & Red-Al (3.4) & THF & 0 & 0.5 & $93: 0: 0: 7$ & - \\
\hline S14 & Red-Al (3.4) & $\mathrm{DMF}^{b}$ & 0 & 0.5 & $15: 11: 0: 74$ & - \\
\hline S15 & Red-Al (3.4) & toluene & 0 & 0.5 & $93: 0: 5: 2$ & 87 \\
\hline S16 & Red-Al (3.4) & hexane & 0 & 0.5 & $57: 2: 0: 41$ & - \\
\hline S17 & Red-Al $(3.4)^{c}$ & $\mathrm{CH}_{2} \mathrm{Cl}_{2}$ & 0 & 0.5 & $97: 0: 0: 3$ & 89 \\
\hline $\mathrm{S} 18$ & Red-Al (3.4) & $\mathrm{CH}_{2} \mathrm{Cl}_{2}$ & 0 & 3 & 96:0:3:1 & - \\
\hline
\end{tabular}

${ }^{a}$ Determined by ${ }^{1} \mathrm{H}$ NMR. ${ }^{b}$ A similar result was obtained with DMA. ${ }^{c}$ The reaction was completed with 2.5 equiv of Red-Al and produced 21 with a ratio of 97:0:0:3. 


\section{Experimental}

\section{General}

The ${ }^{1} \mathrm{H}$ (300 or $400 \mathrm{MHz}$ ) and ${ }^{13} \mathrm{C}$ NMR (75 or $100 \mathrm{MHz}$ ) spectroscopic data were recorded in $\mathrm{CDCl}_{3}$ using $\mathrm{Me}_{4} \mathrm{Si}(\delta=0 \mathrm{ppm})$ and the centreline of the triplet $(\delta=77.1 \mathrm{ppm})$, respectively, as internal standards. Signal patterns are indicated as br s (broad singlet), s (singlet), d (doublet), $\mathrm{t}$ (triplet), q (quartet) and $\mathrm{m}$ (multiplet). Coupling constants $(J)$ are given in Hertz (Hz). Chemical shifts of carbons are accompanied by minus (for $\mathrm{C}$ and $\mathrm{CH}_{2}$ ) and plus (for $\mathrm{CH}$ and $\mathrm{CH}_{3}$ ) signs of the attached proton test (APT) experiments. High-resolution mass spectroscopy (HRMS) was performed with a double-focusing mass spectrometer with an ionization mode of positive FAB. The solvents that were distilled prior to use are THF (from $\mathrm{Na}$ /benzophenone), $\mathrm{Et}_{2} \mathrm{O}$ (from $\mathrm{Na}$ /benzophenone) and $\mathrm{CH}_{2} \mathrm{Cl}_{2}$ (from $\mathrm{CaH}_{2}$ ). Products were purified by chromatography on silica gel (Kanto, spherical silica gel 60N).

\section{A Preliminary 1,4-Addition (Scheme 2, eq 4 in the text)}

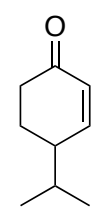<smiles>COc1ccccc1Br</smiles>
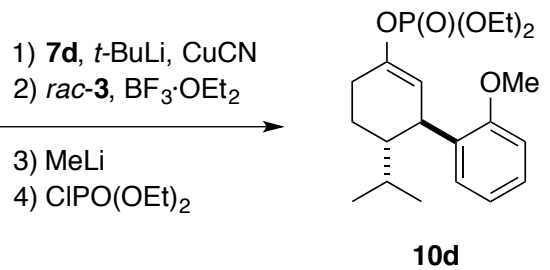

rac-3

To an ice-cold solution of 1-bromo-2-methoxybenzene (7d) (162 mg, $0.866 \mathrm{mmol}^{2}$ in $\mathrm{Et}_{2} \mathrm{O}$ $(2 \mathrm{~mL})$ was added $t$-BuLi (1.61 $\mathrm{M}$ in $n$-pentane, $1.08 \mathrm{~mL}, 1.74 \mathrm{mmol})$ dropwise. The solution was stirred at $0{ }^{\circ} \mathrm{C}$ for $1 \mathrm{~h}$ and added to a slurry of $\mathrm{CuCN}(38 \mathrm{mg}, 0.43 \mathrm{mmol})$. The resulting mixture was stirred for $40 \mathrm{~min}$ at $0{ }^{\circ} \mathrm{C}$ and cooled to $-70{ }^{\circ} \mathrm{C}$. A solution of enone $\mathrm{rac}-3 \mathrm{~m}(40 \mathrm{mg}$, $0.29 \mathrm{mmol})$ in $\mathrm{Et}_{2} \mathrm{O}(1 \mathrm{~mL})$ was added to the mixture, which was warm up to $0{ }^{\circ} \mathrm{C}$ over $5 \mathrm{~h}$ before addition of $\mathrm{ClPO}(\mathrm{OEt})_{2}(0.165 \mathrm{~mL}, 1.15 \mathrm{mmol})$. The mixture was stirred at $\mathrm{rt}$ overnight and diluted with saturated $\mathrm{NH}_{4} \mathrm{Cl}$. The resulting mixture was extracted with EtOAc twice. The combined extracts were washed with $\mathrm{NaHCO}_{3}$ and brine, dried over $\mathrm{MgSO}_{4}$, and concentrated to give a residue, which was purified by chromatography (hexane/EtOAc) to afford 10d (82 mg, 74\% yield): $R_{\mathrm{f}} 0.15$ (hexane/EtOAc 3:1); IR (neat) $1685,1271,1243,1033 \mathrm{~cm}^{-1} ;{ }^{1} \mathrm{H}$ $\operatorname{NMR}\left(300 \mathrm{MHz}, \mathrm{CDCl}_{3}\right) \delta 0.85(\mathrm{~d}, J=6.9 \mathrm{~Hz}, 3 \mathrm{H}), 0.92(\mathrm{~d}, J=6.9 \mathrm{~Hz}, 3 \mathrm{H}), 1.34(\mathrm{t}, J=7.1$ $\mathrm{Hz}, 6 \mathrm{H}), 1.28-1.44(\mathrm{~m}, 1 \mathrm{H}), 1.46-1.79$ (m, $3 \mathrm{H}), 2.26-2.36(\mathrm{~m}, 2 \mathrm{H}), 3.81(\mathrm{~s}, 3 \mathrm{H}), 3.91-4.00$ $(\mathrm{m}, 1 \mathrm{H}), 4.08-4.23(\mathrm{~m}, 4 \mathrm{H}), 5.28-5.34(\mathrm{~m}, 1 \mathrm{H}), 6.84(\mathrm{dd}, J=8.4,1.2 \mathrm{~Hz}, 1 \mathrm{H}), 6.91(\mathrm{dt}, J=$ $1.2,7.4 \mathrm{~Hz}, 1 \mathrm{H}), 7.13-7.22(\mathrm{~m}, 2 \mathrm{H}) \mathrm{ppm} ;{ }^{13} \mathrm{C}-\mathrm{APT}$ NMR $\left(75 \mathrm{MHz}, \mathrm{CDCl}_{3}\right) \delta 16.2(+)(\mathrm{d}, J$ $=7 \mathrm{~Hz}), 17.9(+), 21.1(-), 21.7(+), 26.9(-)(\mathrm{d}, J=4 \mathrm{~Hz}), 27.3(+), 36.1(+), 45.6(+), 55.2$ $(+), 64.1(-)(\mathrm{d}, J=6 \mathrm{~Hz}), 110.2(+), 114.4(+)(\mathrm{d}, J=6 \mathrm{~Hz}), 120.5(+), 127.2(+), 129.1(+)$, $133.3(-), 118.3(-)(\mathrm{d}, J=9 \mathrm{~Hz}), 157.2(-)$ ppm; HRMS (FAB) calcd for $\mathrm{C}_{20} \mathrm{H}_{32} \mathrm{O}_{5} \mathrm{P}$ $\left[(\mathrm{M}+\mathrm{H})^{+}\right]$383.1987, found 383.1982. 


\section{Synthesis of Monoterpenylmagnolol}

\section{(S)-4-Isopropylcyclohex-2-en-1-one (3)}

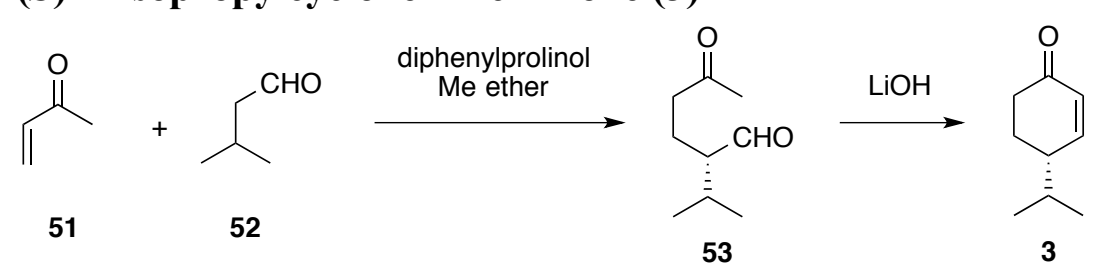

To an ice-cold mixture of isovaleraldehyde (52) $(3.20 \mathrm{~mL}, 29.7 \mathrm{mmol})$, diphenylprolinol methyl ether (397 mg, $1.48 \mathrm{mmol})$, and ethyl 3,4-dihydroxybenzoate (1.08 g, $5.93 \mathrm{mmol})$ was added methyl vinyl ketone $51(3.64 \mathrm{~mL}, 44.6 \mathrm{mmol})$. The mixture was stirred at $4{ }^{\circ} \mathrm{C}$ for 6 days and concentrated to afford a residue, which was purified by chromatography on silica gel to give the Michael addition product 53 ( $3.15 \mathrm{~g}, 68 \%$ yield).

To a solution of the above product $\mathbf{5 3}(1.21 \mathrm{~g}, 7.77 \mathrm{mmol})$ in $i-\mathrm{PrOH}(78 \mathrm{~mL})$ was added $\mathrm{LiOH}(186 \mathrm{mg}, 7.77 \mathrm{mmol})$. The mixture was stirred at $\mathrm{rt}$ for $6 \mathrm{~h}$ and diluted with $\mathrm{NH}_{4} \mathrm{Cl}$. The resulting mixture was extracted with $\mathrm{Et}_{2} \mathrm{O}$ twice. The combined ethereal solutions were dried over $\mathrm{MgSO}_{4}$ and concentrated. The residue was purified by chromatography on silica gel (hexane/EtOAc) to afford enone 3 ( $1.07 \mathrm{~g}, 52 \%$ yield): $84 \%$ ee by chiral HPLC analysis (Chiralcel AD-H, hexane $/ i-\mathrm{PrOH}=99.5 / 0.5,31{ }^{\circ} \mathrm{C}, 1 \mathrm{~mL} / \mathrm{min}, t_{\mathrm{R}}(\min )=15.1$ (major $(S)$-isomer), 21.0 (minor $(R)$-isomer)); $R_{\mathrm{f}} 0.41$ (hexane/EtOAc 5:1); ${ }^{1} \mathrm{H}$ NMR $(300 \mathrm{MHz}$, $\left.\mathrm{CDCl}_{3}\right) \delta 0.96(\mathrm{~d}, J=6.9 \mathrm{~Hz}, 3 \mathrm{H}), 0.98(\mathrm{~d}, J=6.6 \mathrm{~Hz}, 3 \mathrm{H}), 1.70-1.89(\mathrm{~m}, 2 \mathrm{H}), 1.96-2.06$ $(\mathrm{m}, 1 \mathrm{H}), 2.26-2.36(\mathrm{~m}, 1 \mathrm{H}), 2.38(\mathrm{dd}, J=13.5,4.8 \mathrm{~Hz}, 1 \mathrm{H}), 2.52(\mathrm{dt}, J=16.5,4.2 \mathrm{~Hz}, 1 \mathrm{H})$, 6.02 (ddd, $J=10.3,2.4,0.9 \mathrm{~Hz}, 1 \mathrm{H}), 6.91$ (ddd, $J=10.3,2.4,1.8 \mathrm{~Hz}, 1 \mathrm{H}) ;{ }^{13} \mathrm{C}-$ APT NMR $\left(75 \mathrm{MHz}, \mathrm{CDCl}_{3}\right)$ \& $19.5(+), 19.6(+), 25.2(-), 31.5(+), 37.4(-), 42.5(+), 129.7(+), 154.6$ $(+), 200.3(+)$. The ${ }^{1} \mathrm{H}$ and ${ }^{13} \mathrm{C}$ NMR spectra were consistent with those reported. ${ }^{\mathrm{S} 2}$

Note that the ee was increased at a later stage to $95 \%$.

\section{2,2'-Bis(methoxymethoxy)-1,1'-biphenyl (55)}

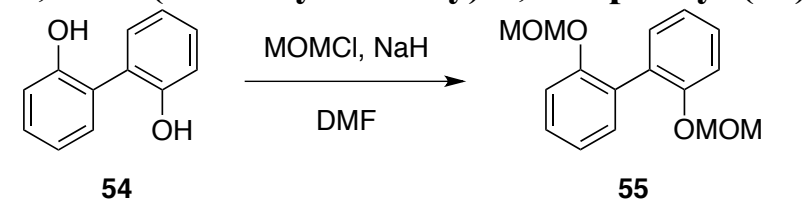

To an ice-cold solution of $\mathrm{NaH}(55 \%$ in mineral oil, $3.75 \mathrm{~g}, 85.9 \mathrm{mmol})$ in DMF (20 mL) was added a solution of 2,2'-biphenol (54) $(4.03 \mathrm{~g}, 21.6 \mathrm{mmol})$ in DMF $(10 \mathrm{~mL})$. After stirring for $30 \mathrm{~min}, \mathrm{MOMCl}(5.70 \mathrm{~mL}, 75.8 \mathrm{mmol})$ was added. The mixture was stirred at room temperature for $2 \mathrm{~h}$ and diluted with saturated $\mathrm{NaHCO}_{3}$. The resulting mixture was extracted with $\mathrm{Et}_{2} \mathrm{O}$ twice. The combined ethereal solutions were washed with brine, dried over $\mathrm{MgSO}_{4}$, and concentrated to give a residue, which was purified by chromatography (hexane/EtOAc) to afford 55 (5.93 g, 100\% yield): $R_{\mathrm{f}} 0.33$ (hexane/EtOAc 8:1); ${ }^{1} \mathrm{H}$ NMR (300 MHz, $\left.\mathrm{CDCl}_{3}\right) \delta$ $3.34(\mathrm{~s}, 6 \mathrm{H}), 5.07(\mathrm{~s}, 4 \mathrm{H}), 7.07$ (dt, $J=1.2,7.2 \mathrm{~Hz}, 2 \mathrm{H}), 7.19-7.35(\mathrm{~m}, 6 \mathrm{H}) ;{ }^{13} \mathrm{C}-$ APT NMR $\left(75 \mathrm{MHz}, \mathrm{CDCl}_{3}\right) \delta 55.8(+), 95.2(-), 115.5(+), 121.8(+), 128.7(+), 129.2(-), 131.5(+)$, $154.9(-)$. The ${ }^{1} \mathrm{H}$ and ${ }^{13} \mathrm{C}$ NMR spectra were consistent with those reported. ${ }^{\mathrm{S} 3}$

\section{3-Bromo-2,2'-bis(methoxymethoxy)-1,1'-biphenyl (7e)}




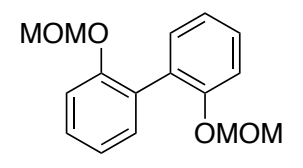

55

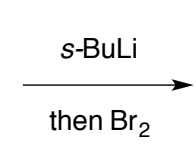

hen $\mathrm{Br}_{2}$

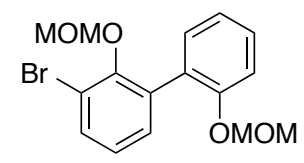

$7 e$

To a solution of the diether $\mathbf{5 5}(2.87 \mathrm{~g}, 10.5 \mathrm{mmol})$ in THF (100 mL) was added sec-BuLi (1.02 $\mathrm{M}$ in cyclohexane and $n$-hexane, $12.3 \mathrm{~mL}, 12.5 \mathrm{mmol}$ ) dropwise at $-78^{\circ} \mathrm{C}$. The solution was stirred for $30 \mathrm{~min}$ and $\mathrm{Br}_{2}(0.70 \mathrm{~mL}, 13.7 \mathrm{mmol})$ was added to the solution. The mixture was stirred at $-78^{\circ} \mathrm{C}$ for $2 \mathrm{~h}$ and poured into saturated aqueous $\mathrm{Na}_{2} \mathrm{~S}_{2} \mathrm{O}_{3}$. The resulting mixture was extracted with EtOAc twice. The combined organic layers were dried over $\mathrm{MgSO}_{4}$ and concentrated to give a residue, which was purified by chromatography (hexane/EtOAc) to afford $7 \mathrm{e}\left(2.88 \mathrm{~g}, 78 \%\right.$ yield): $R_{\mathrm{f}} 0.43$ (hexane/EtOAc $\left.8: 1\right) ;{ }^{1} \mathrm{H}$ NMR $\left(300 \mathrm{MHz}, \mathrm{CDCl}_{3}\right) \delta$ $3.01(\mathrm{~s}, 3 \mathrm{H}), 3.37(\mathrm{~s}, 3 \mathrm{H}), 4.76(\mathrm{~s}, 2 \mathrm{H}), 5.11(\mathrm{~s}, 2 \mathrm{H}), 7.05(\mathrm{t}, J=7.8 \mathrm{~Hz}, 1 \mathrm{H}), 7.08(\mathrm{dd}, J=$ 7.1, $0.9 \mathrm{~Hz}, 1 \mathrm{H}), 7.17-7.36(\mathrm{~m}, 4 \mathrm{H}), 7.57(\mathrm{dd}, J=7.8,1.6 \mathrm{~Hz}, 1 \mathrm{H}) ;{ }^{13} \mathrm{C}-\mathrm{APT}$ NMR $(75$ $\left.\mathrm{MHz}, \mathrm{CDCl}_{3}\right) \delta 56.0(+), 57.0(+), 94.8(-), 99.2(-), 115.2(+), 117.7(-), 121.8(+), 125.1(+)$, $128.4(-), 129.2(+), 131.1(+), 131.7(+), 132.7(+), 134.7(-), 152.1(-), 154.6(-)$; HRMS (FAB) calcd for $\mathrm{C}_{16} \mathrm{H}_{17} \mathrm{BrO}_{4}\left[\mathrm{M}^{+}\right]$352.0310, found 352.0313 .

Diethyl((1S,6S)-6-isopropyl-2',2'-bis(methoxymethoxy)-1,4,5,6-tetrahydro-[1,1':3',1'"-ter phenyl]-3-yl) phosphate (10e)
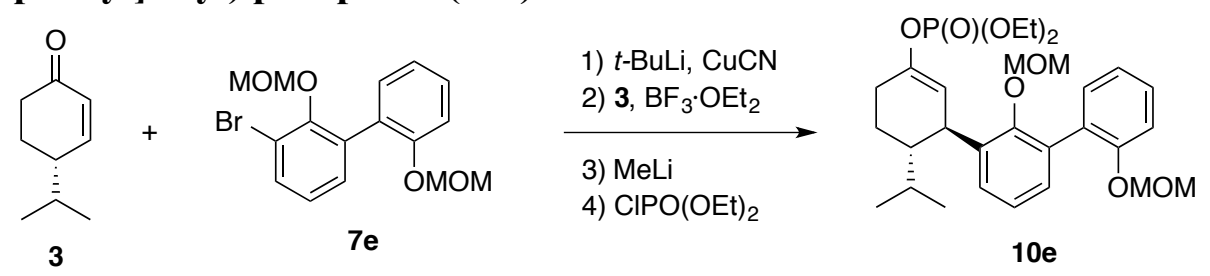

To a solution of the aryl bromide 7e $(1.26 \mathrm{~g}, 3.56 \mathrm{mmol})$ in THF $(24 \mathrm{~mL})$ was added $t$-BuLi (1.77 $\mathrm{M}$ in $n$-pentane, $4.02 \mathrm{~mL}, 7.12 \mathrm{mmol}$ ) dropwise at $-70^{\circ} \mathrm{C}$. After the mixture was stirred for $10 \mathrm{~min}$ at $-70{ }^{\circ} \mathrm{C}$, for $20 \mathrm{~min}$ at $0{ }^{\circ} \mathrm{C}, \mathrm{CuCN}(159 \mathrm{mg}, 1.78 \mathrm{mmol})$ was added to the solution. The mixture was stirred for $20 \mathrm{~min}$ at $0{ }^{\circ} \mathrm{C}$ and cooled to $-70{ }^{\circ} \mathrm{C}$. The enone $3(84 \%$ ee, $164 \mathrm{mg}, 1.19 \mathrm{mmol})$ and $\mathrm{BF}_{3} \cdot \mathrm{Et}_{2} \mathrm{O}(0.300 \mathrm{~mL}, 2.39 \mathrm{mmol})$ in $\mathrm{THF}(12 \mathrm{~mL})$ were added to the mixture over $5 \mathrm{~min}$ at $-70{ }^{\circ} \mathrm{C}$. The reaction mixture was warm up to $-50{ }^{\circ} \mathrm{C}$ over $1.5 \mathrm{~h}$. $\mathrm{MeLi}\left(6.30 \mathrm{~mL}, 1.13 \mathrm{M}\right.$ in $\left.\mathrm{Et}_{2} \mathrm{O}, 7.12 \mathrm{mmol}\right)$ was added to the solution. After stirred for 30 min at $0{ }^{\circ} \mathrm{C}, \mathrm{ClPO}(\mathrm{OEt})_{2}(0.850 \mathrm{~mL}, 5.91 \mathrm{mmol})$ was added at $0{ }^{\circ} \mathrm{C}$. After $1 \mathrm{~h}$ at $\mathrm{rt}$, the solution was poured into saturated $\mathrm{NH}_{4} \mathrm{Cl}$. The resulting mixture was extracted with $\mathrm{Et}_{2} \mathrm{O}$ twice. The combined extracts were washed with $\mathrm{NaHCO}_{3}$ and brine, dried over $\mathrm{MgSO}_{4}$, and concentrated to give a residue, which was purified by chromatography (hexane/EtOAc) to afford 10e (473 mg, 73\% yield): $[\alpha]_{\mathrm{D}}{ }^{19}-46\left(c 0.65, \mathrm{CHCl}_{3}\right) ; R_{\mathrm{f}} 0.26$ (hexane/EtOAc $\left.2: 1\right) ;{ }^{1} \mathrm{H}$ $\operatorname{NMR}\left(400 \mathrm{MHz}, \mathrm{CDCl}_{3}\right) \delta 0.87(\mathrm{~d}, J=6.8 \mathrm{~Hz}, 3 \mathrm{H}), 0.89(\mathrm{~d}, J=6.8 \mathrm{~Hz}, 3 \mathrm{H}), 1.35(\mathrm{t}, J=7.2$ $\mathrm{Hz}, 6 \mathrm{H}), 1.46-1.76(\mathrm{~m}, 3 \mathrm{H}), 1.80-1.88(\mathrm{~m}, 1 \mathrm{H}), 2.30-2.50(\mathrm{~m}, 2 \mathrm{H}), 3.08$ (s, $3 \mathrm{H}), 3.38$ (s, 3 H), 3.92-3.99 (m, $1 \mathrm{H}), 4.15(\mathrm{q}, J=7.2 \mathrm{~Hz}, 2 \mathrm{H}), 4.17(\mathrm{q}, J=7.2 \mathrm{~Hz}, 2 \mathrm{H}), 4.48(\mathrm{~d}, J=5.8 \mathrm{~Hz}$, $1 \mathrm{H}), 4.59(\mathrm{~d}, J=5.8 \mathrm{~Hz}, 1 \mathrm{H}), 5.02-5.12(\mathrm{~m}, 2 \mathrm{H}), 5.36-5.40(\mathrm{~m}, 1 \mathrm{H}), 7.03-7.09(\mathrm{~m}, 1 \mathrm{H})$, 7.10-7.16 (m, $2 \mathrm{H}), 7.17-7.26(\mathrm{~m}, 2 \mathrm{H}), 7.26-7.32(\mathrm{~m}, 2 \mathrm{H}) ;{ }^{13} \mathrm{C}-\mathrm{APT}$ NMR $(100 \mathrm{MHz}$, $\left.\mathrm{CDCl}_{3}\right) \delta 16.1(+), 16.2(+), 17.2(+), 21.7(+), 21.9(-), 27.3(+), 27.9(-), 37.1(+), 46.8(+)$, $55.9(+), 57.0(+), 64.1(\mathrm{~d}, J=6 \mathrm{~Hz}, 2 \mathrm{C})(-), 94.9(-), 99.5(-), 115.2(+), 115.3(\mathrm{~d}, J=5 \mathrm{~Hz})$ $(+), 121.8(+), 124.2(+), 128.3(+), 128.8(+), 129.7(+), 131.6(+), 132.2(-), 138.3(-), 148.2$ $(\mathrm{d}, J=9 \mathrm{~Hz})(-), 153.5(-), 154.8(-)$; HRMS (FAB) calcd for $\mathrm{C}_{29} \mathrm{H}_{42} \mathrm{O}_{8} \mathrm{P}\left[(\mathrm{M}+\mathrm{H})^{+}\right]$ 
549.2617, found 549.2616.

(1S,2S)-2-Isopropyl-2',2"'-bis(methoxymethoxy)-5-methyl-1,2,3,4-tetrahydro-1,1':3',1'"-te
rphenyl (12)
$\mathrm{OP}(\mathrm{O})(\mathrm{OEt})_{2}$

To an ice-cold solution of $\mathrm{Ni}(\mathrm{acac})_{2}(7 \mathrm{mg}, 0.027 \mathrm{mmol})$ in THF $(5 \mathrm{~mL})$ was added $\mathrm{MeMgCl}(3.0 \mathrm{M}$ in THF, $0.17 \mathrm{~mL}, 0.51 \mathrm{mmol})$. After $10 \mathrm{~min}$ at $0{ }^{\circ} \mathrm{C}$, a solution of enol phosphate 10e $(69 \mathrm{mg}, 0.126 \mathrm{mmol})$ in THF $(1 \mathrm{~mL})$ was added. The solution was stirred at room temperature for $3 \mathrm{~h}$ and diluted with saturated $\mathrm{NH}_{4} \mathrm{Cl}$. The resulting mixture was extracted with EtOAc twice. The combined organic layers were washed with brine, dried over $\mathrm{MgSO}_{4}$, and concentrated to give a residue, which was purified by chromatography (hexane/EtOAc) to afford 12 (47 mg, 91\% yield, 84\% ee by chiral HPLC analysis), which was subjected to recrystallization from hexane to afford $95 \%$ ee of $\mathbf{3 3}$ from the mother liquor (36 mg, 70\% yield based on 10e, 77\% recovery): chiral HPLC analysis (Chiralcel AD-H, hexane $/ i-\mathrm{PrOH}=99.5 / 0.5,27^{\circ} \mathrm{C}, 0.5 \mathrm{~mL} / \mathrm{min}, t_{\mathrm{R}}(\mathrm{min})=16.0$ (major isomer), 17.3 (minor isomer)); $[\alpha]_{\mathrm{D}}{ }^{19}-81\left(c 1.00, \mathrm{CHCl}_{3}\right.$ ) for $95 \%$ ee; $R_{\mathrm{f}} 0.80$ (hexane/EtOAc $\left.1: 1\right) ;{ }^{1} \mathrm{H}$ NMR (400 $\left.\mathrm{MHz}, \mathrm{CDCl}_{3}\right) \delta 0.85(\mathrm{~d}, J=7.0 \mathrm{~Hz}, 3 \mathrm{H}), 0.87(\mathrm{~d}, J=7.0 \mathrm{~Hz}, 3 \mathrm{H}), 1.37-1.66(\mathrm{~m}, 3 \mathrm{H}), 1.71$ (s, $3 \mathrm{H}), 1.72-1.81(\mathrm{~m}, 1 \mathrm{H}), 1.98-2.19(\mathrm{~m}, 2 \mathrm{H}), 3.04(\mathrm{~s}, 3 \mathrm{H}), 3.39$ (s, $3 \mathrm{H}), 3.74-3.82(\mathrm{~m}, 1$ $\mathrm{H}), 4.50(\mathrm{~d}, J=5.8 \mathrm{~Hz}, 1 \mathrm{H}), 4.62(\mathrm{~d}, J=5.8 \mathrm{~Hz}, 1 \mathrm{H}), 5.06(\mathrm{~d}, J=6.8 \mathrm{~Hz}, 1 \mathrm{H}), 5.10(\mathrm{~d}, J=$ $6.8 \mathrm{~Hz}, 1 \mathrm{H}), 5.26(\mathrm{~s}, 1 \mathrm{H}), 7.06(\mathrm{dt}, J=0.8,7.2 \mathrm{~Hz}, 1 \mathrm{H}), 7.09-7.15(\mathrm{~m}, 2 \mathrm{H}), 7.16-7.22$ (m, 2 $\mathrm{H}), 7.26-7.33(\mathrm{~m}, 2 \mathrm{H}) ;{ }^{13} \mathrm{C}-\mathrm{APT}$ NMR $\left(75 \mathrm{MHz}, \mathrm{CDCl}_{3}\right) \delta 17.0(+), 21.7(+), 22.2(-), 23.6$

$(+), 27.7(+), 30.6(-), 38.6(+), 47.2(+), 55.9(+), 56.9(+), 94.8(-), 99.5(-), 115.2(+), 121.8$ $(+), 124.1(+), 126.0(+), 128.4(+), 128.7(+), 129.1(+), 129.9(-), 131.6(+), 132.3(-), 134.1$ $(-), 139.7(-), 153.6(-), 154.8(-)$; HRMS (FAB) calcd for $\mathrm{C}_{26} \mathrm{H}_{34} \mathrm{O}_{4}\left[\mathrm{M}^{+}\right]$410.2457, found 410.2460 .

This product $\mathbf{1 2}$ of $95 \%$ ee was used for the subsequent transformations.

$(1 " S, 2 " S)-2 "$-Isopropyl-5"'-methyl-1",2",3",4"-tetrahydro-[1,1':3',1"'-terphenyl]-2,2'-dio l (13)<smiles>COc1ccccc1-c1cccc([C@@H]2C=C(C)CC[C@H]2C(C)C)c1OC</smiles>

12

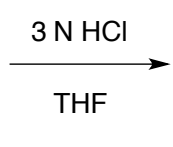

To a solution of the MOM ether $12(539 \mathrm{mg}, 1.31 \mathrm{mmol})$ in THF $(50 \mathrm{~mL})$ was added $3 \mathrm{~N}$ $\mathrm{HCl}(16.5 \mathrm{~mL})$. After refluxing for $6 \mathrm{~h}$ at $80^{\circ} \mathrm{C}$, the mixture was cooled to $0{ }^{\circ} \mathrm{C}$ and diluted with saturated $\mathrm{NaHCO}_{3}$. The being heated under reflux, the mixture was extracted with $\mathrm{Et}_{2} \mathrm{O}$ twice. The combined ethereal layers were washed with brine, dried over $\mathrm{MgSO}_{4}$, and concentrated to give a residue, which was semi-purified by chromatography (hexane/EtOAc) to furnish biphenol 13, which was used for the next reaction without further purification.

The reaction was carried out again to afford $\mathbf{1 3}$, which was purified by chromatography on 
silica gel (hexane/EtOAc) for analysis: $[\alpha]_{\mathrm{D}}{ }^{18}-94\left(c 0.96, \mathrm{CHCl}_{3}\right) ; R_{\mathrm{f}} 0.32$ (hexane/EtOAc 6:1); ${ }^{1} \mathrm{H}$ NMR $\left(300 \mathrm{MHz}, \mathrm{CDCl}_{3}\right) \delta 0.86(\mathrm{~d}, J=6.8 \mathrm{~Hz}, 3 \mathrm{H}), 0.89$ (d, $\left.J=6.8 \mathrm{~Hz}, 3 \mathrm{H}\right), 1.33-$ $1.49(\mathrm{~m}, 1 \mathrm{H}), 1.54-1.72(\mathrm{~m}, 2 \mathrm{H}), 1.76(\mathrm{~s}, 3 \mathrm{H}), 1.77-1.84(\mathrm{~m}, 1 \mathrm{H}), 2.06-2.26(\mathrm{~m}, 2 \mathrm{H})$, $3.48-3.58(\mathrm{~m}, 1 \mathrm{H}), 5.48-5.54(\mathrm{~m}, 1 \mathrm{H}), 5.91(\mathrm{~s}, 1 \mathrm{H}), 6.20(\mathrm{~s}, 1 \mathrm{H}), 6.98(\mathrm{t}, J=7.5 \mathrm{~Hz}, 1 \mathrm{H})$, 7.02-7.07 (m, $2 \mathrm{H}), 7.14$ (dd, $J=7.5,1.8 \mathrm{~Hz}, 1 \mathrm{H}), 7.17$ (dd, $J=7.5,1.8 \mathrm{~Hz}, 1 \mathrm{H}), 7.24-7.34$ $(\mathrm{m}, 2 \mathrm{H}) ;{ }^{13} \mathrm{C}-$ APT NMR $\left(75 \mathrm{MHz}, \mathrm{CDCl}_{3}\right) \delta 16.6(+), 21.6(-), 21.7(+), 23.8(+), 27.5(+)$, $30.3(-), 42.2(+), 44.5(+), 117.0(+), 121.1(+), 121.3(+), 124.1(+), 124.9(-), 125.1(-)$, $129.6(+) 129.8(+), 130.8(+), 131.4(+), 131.5(-), 138.9(-), 151.0(-), 153.3(-)$; HRMS (FAB) calcd for $\mathrm{C}_{22} \mathrm{H}_{26} \mathrm{O}_{2}\left[\mathrm{M}^{+}\right] 322.1933$, found 322.1948 .

2-((9S,9aR)-2,6-Diiodo-9-isopropyl-6-methyl-5a,6,7,8,9,9a-hexahydrodibenzo[b, $d]$ furan-4 -yl)-4,6-diiodophenol (14)

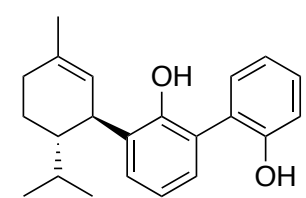

13
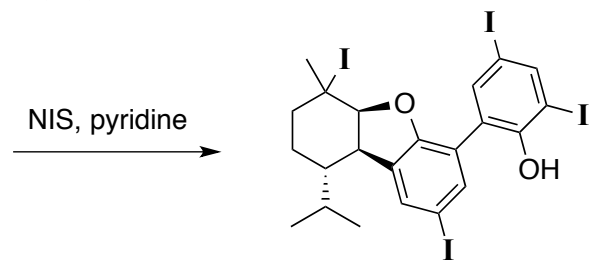

14

To an ice-cold solution of biphenol 13 obtained above in $\mathrm{MeCN}(40 \mathrm{~mL})$ and pyridine (4 $\mathrm{mL}$ ) was added NIS $(1.91 \mathrm{~g}, 8.49 \mathrm{mmol})$. After being stirred at $0{ }^{\circ} \mathrm{C}$ for $2 \mathrm{~h}$, the mixture was diluted with saturated $\mathrm{NaHCO}_{3}$ and aqueous $\mathrm{Na}_{2} \mathrm{~S}_{2} \mathrm{O}_{3}$. The resulting mixture was extracted with hexane twice. The combined organic layers were dried over $\mathrm{MgSO}_{4}$ and concentrated to give a residue, which was purified by chromatography (hexane/EtOAc) to furnish furan $\mathbf{1 4}$ (802 mg, 74\% yield from the MOM ether 12): $R_{\mathrm{f}} 0.55$ (hexane/EtOAc 8:1); ${ }^{1} \mathrm{H}$ NMR (300 $\left.\mathrm{MHz}, \mathrm{CDCl}_{3}\right) \delta 0.89$ (d, $\left.J=6.9 \mathrm{~Hz}, 3 \mathrm{H}\right), 1.02(\mathrm{~d}, J=6.9 \mathrm{~Hz}, 3 \mathrm{H}), 1.02-1.17(\mathrm{~m}, 1 \mathrm{H}), 1.22-$ $1.36(\mathrm{~m}, 1 \mathrm{H}), 1.57-1.75(\mathrm{~m}, 2 \mathrm{H}), 1.86-2.00(\mathrm{~m}, 1 \mathrm{H}), 2.17(\mathrm{~d}, J=15.0 \mathrm{~Hz}, 1 \mathrm{H}), 2.30(\mathrm{~s}, 3$ H), $3.34(\mathrm{dd}, J=11.5,4.8 \mathrm{~Hz}, 1 \mathrm{H}), 4.98(\mathrm{~d}, J=4.8 \mathrm{~Hz}, 1 \mathrm{H}), 6.83(\mathrm{~s}, 1 \mathrm{H}), 7.49-7.54(\mathrm{~m}, 2$ $\mathrm{H}), 7.58(\mathrm{~d}, J=2.1 \mathrm{~Hz}, 1 \mathrm{H}), 8.03(\mathrm{~d}, J=2.4 \mathrm{~Hz}, 1 \mathrm{H}) ;{ }^{13} \mathrm{C}-\mathrm{APT}$ NMR $\left(75 \mathrm{MHz}, \mathrm{CDCl}_{3}\right) \delta$ $15.5(+), 21.6(+), 22.3(-), 26.0(+), 34.3(+), 39.3(-), 42.0(+), 44.7(+), 51.1(-), 83.6(-)$, $84.2(-), 89.0(-), 93.2(+), 121.7(-), 125.3(-), 133.7(+), 136.7(-), 137.9(+), 139.5(+)$, 146.2 (+), $152.4(-), 154.1(-)$; HRMS (FAB) calcd for $\mathrm{C}_{22} \mathrm{H}_{22} \mathrm{O}_{2} \mathrm{I}_{4}\left[\mathrm{M}^{+}\right]$825.7799, found 825.7785 .

(1S,2S)-2"-((tert-Butyldimethylsilyl)oxy)-5',5"'diiodo-2-isopropyl-5-methyl-1,2,3,4-tetrah ydro-[1,1':3',1'-terphenyl]-2'-ol (18) 


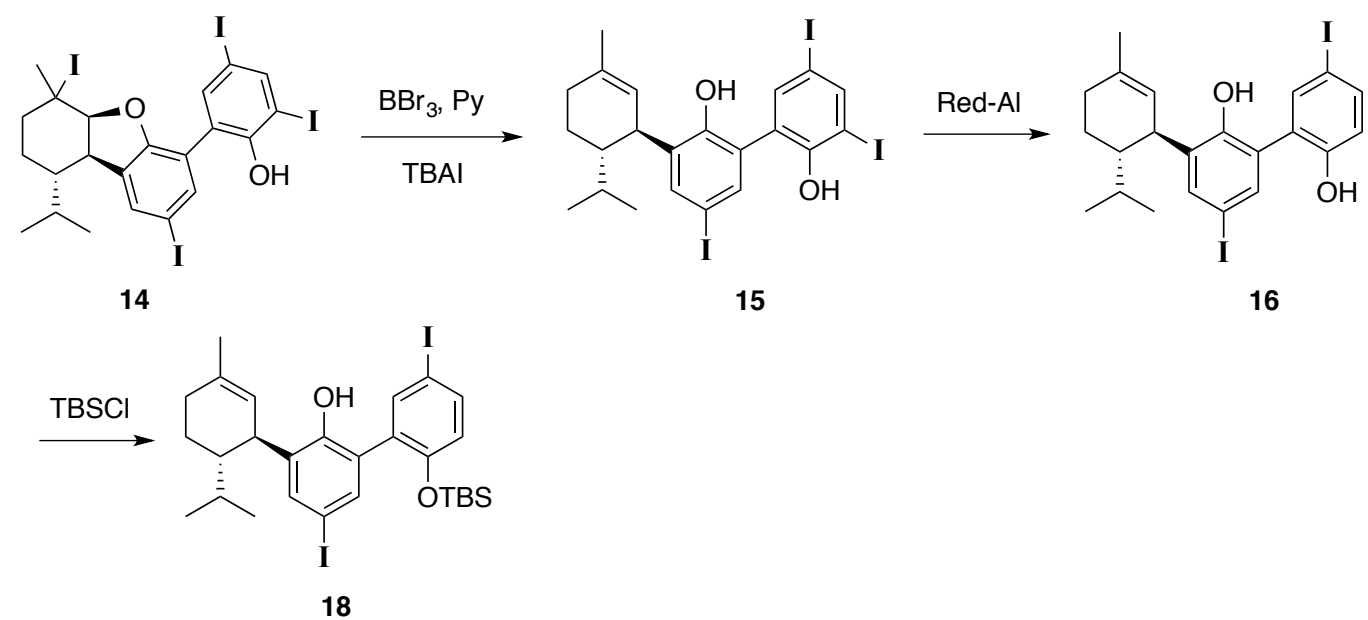

To a solution of furan $14(197 \mathrm{mg}, 0.238 \mathrm{mmol})$ in $\mathrm{CH}_{2} \mathrm{Cl}_{2}(10 \mathrm{~mL})$ were added pyridine $(0.058 \mathrm{~mL}, 0.72 \mathrm{mmol})$, tetrabutylammonium iodide $(528 \mathrm{mg}, 1.43 \mathrm{mmol})$, and $\mathrm{BBr}_{3}(1.0 \mathrm{M}$ in $\left.\mathrm{CH}_{2} \mathrm{Cl}_{2}, 1.20 \mathrm{~mL}, 1.20 \mathrm{mmol}\right)$ at $-15^{\circ} \mathrm{C}$. The mixture was stirred at $-10{ }^{\circ} \mathrm{C}$ for $3 \mathrm{~h}$ and diluted with $\mathrm{NaHCO}_{3}$ and aqueous $\mathrm{Na}_{2} \mathrm{~S}_{2} \mathrm{O}_{3}(1: 1)$. The resulting mixture was extracted with $\mathrm{CH}_{2} \mathrm{Cl}_{2}$ three times. The combined extracts were dried over $\mathrm{MgSO}_{4}$ and concentrated to give 15, which was used for the next reaction without further purification: $R_{\mathrm{f}} 0.47$ (hexane/EtOAc 6:1); ${ }^{1} \mathrm{H}$ NMR $\left(300 \mathrm{MHz}, \mathrm{CDCl}_{3}\right) \delta 0.85(\mathrm{~d}, J=6.9 \mathrm{~Hz}, 3 \mathrm{H}), 0.89(\mathrm{~d}, J=6.9 \mathrm{~Hz}, 3 \mathrm{H}), 1.31-1.69$ $(\mathrm{m}, 3 \mathrm{H}), 1.70-1.84(\mathrm{~m}, 1 \mathrm{H}), 1.77(\mathrm{~s}, 3 \mathrm{H}), 2.04-2.16(\mathrm{~m}, 2 \mathrm{H}), 3.38-3.51(\mathrm{~m}, 1 \mathrm{H}), 5.45(\mathrm{~s}, 1$ H), 5.66-6.80 (br s, 2 H), 7.39-7.43 (m, $2 \mathrm{H}), 7.52(\mathrm{~d}, J=2.4 \mathrm{~Hz}, 1 \mathrm{H}), 8.03(\mathrm{~d}, J=2.4 \mathrm{~Hz}, 1$ $\mathrm{H})$; HRMS (FAB) calcd for $\mathrm{C}_{22} \mathrm{H}_{24} \mathrm{O}_{2} \mathrm{I}_{2}\left[\mathrm{M}^{+}\right]$573.9866, found 573.9886.

To an ice-cold solution of the above triiodide 15 in $\mathrm{CH}_{2} \mathrm{Cl}_{2}(7 \mathrm{~mL})$ was added Red-Al (3.6 $\mathrm{M}$ in toluene, $0.33 \mathrm{~mL}, 1.19 \mathrm{mmol}$ ) dropwise. The mixture was stirred at $0{ }^{\circ} \mathrm{C}$ for $1 \mathrm{~h}$ and diluted with $3 \mathrm{~N} \mathrm{HCl}$ carefully. The resulting mixture was extracted with $\mathrm{CH}_{2} \mathrm{Cl}_{2}$ three times. The combined organic layers were washed successively with $\mathrm{NaHCO}_{3}$ and brine, dried over $\mathrm{MgSO}_{4}$, and concentrated to afford diiodide 16, which was used for the next reaction without further purification.

The same transformation was carried out again to afford 16, which was purified by chromatography on silica gel (hexane/EtOAc) for analysis: $[\alpha]_{\mathrm{D}}{ }^{19}-45\left(c 1.13, \mathrm{CHCl}_{3}\right) ; R_{\mathrm{f}} 0.26$ (hexane/EtOAc 6:1); ${ }^{1} \mathrm{H}$ NMR $\left(300 \mathrm{MHz}, \mathrm{CDCl}_{3}\right) \delta 0.86(\mathrm{~d}, J=6.8 \mathrm{~Hz}, 3 \mathrm{H}), 0.90(\mathrm{~d}, J=6.8$ $\mathrm{Hz}, 3 \mathrm{H}), 1.34-1.48(\mathrm{~m}, 1 \mathrm{H}), 1.49-1.70(\mathrm{~m}, 2 \mathrm{H}), 1.77$ (s, $3 \mathrm{H}), 1.72-1.85$ (m, $1 \mathrm{H}), 2.02-2.20$ (m, $2 \mathrm{H}), 3.38-3.50(\mathrm{~m}, 1 \mathrm{H}), 5.45(\mathrm{~s}, 1 \mathrm{H}), 5.58-6.46(\mathrm{br} \mathrm{s}, 2 \mathrm{H}), 6.80(\mathrm{~d}, J=8.1 \mathrm{~Hz}, 1 \mathrm{H})$, $7.41(\mathrm{~d}, J=2.1 \mathrm{~Hz}, 1 \mathrm{H}), 7.45(\mathrm{~d}, J=2.1 \mathrm{~Hz}, 1 \mathrm{H}), 7.54(\mathrm{~d}, J=2.1 \mathrm{~Hz}, 1 \mathrm{H}), 7.58$ (dd, $J=8.5$, $2.1 \mathrm{~Hz}, 1 \mathrm{H}) ;{ }^{13} \mathrm{C}-\mathrm{APT}$ NMR $\left(75 \mathrm{MHz}, \mathrm{CDCl}_{3}\right) \delta 16.5(+), 21.5(-), 21.7(+), 23.8(+), 27.4$ $(+), 30.2(-), 42.3(+), 44.3(+), 83.4(-), 83.8(-), 119.6(+), 123.0(+), 126.5(-), 126.7(-)$, $134.0(-), 138.1(+), 138.6(+), 139.5(2 \mathrm{C},+), 140.4(-), 150.9(-), 153.2(-)$; HRMS (FAB) calcd for $\mathrm{C}_{22} \mathrm{H}_{24} \mathrm{O}_{2} \mathrm{I}_{2}\left[\mathrm{M}^{+}\right]$573.9866, found 573.9886.

To an ice-cold solution of the above biphenol 16 in $\mathrm{CH}_{2} \mathrm{Cl}_{2}(7 \mathrm{~mL})$ were added $\mathrm{Et}_{3} \mathrm{~N}(0.10$ $\mathrm{mL}, 0.72 \mathrm{mmol})$ and TBSCl $(68 \mathrm{mg}, 0.45 \mathrm{mmol})$. The mixture was stirred at $\mathrm{rt}$ for $10 \mathrm{~h}$ and diluted with saturated $\mathrm{NaHCO}_{3}$. The resulting mixture was extracted with $\mathrm{CH}_{2} \mathrm{Cl}_{2}$ three times. The combined organic layers were washed with brine, dried over $\mathrm{MgSO}_{4}$, and concentrated to give a residue, which was purified by chromatography on silica gel (hexane/EtOAc) to give monophenol 18 (122 mg, 77\% yield from furan 14): $[\alpha]_{\mathrm{D}}{ }^{19}-29\left(c 0.62, \mathrm{CHCl}_{3}\right) ; R_{\mathrm{f}} 0.78$ (hexane/EtOAc 6:1); ${ }^{1} \mathrm{H}$ NMR (300 MHz, $\mathrm{CDCl}_{3}$ ) $\delta-0.06$ (br s, $3 \mathrm{H}$ ), 0.04 (br s, $3 \mathrm{H}$ ), 0.82 (s, 
$9 \mathrm{H}), 0.80-0.86(\mathrm{~m}, 3 \mathrm{H}), 0.90(\mathrm{~d}, J=6.9 \mathrm{~Hz}, 3 \mathrm{H}), 1.37-1.76(\mathrm{~m}, 4 \mathrm{H}), 1.73$ (s, $3 \mathrm{H}), 1.92-$ $2.14(\mathrm{~m}, 2 \mathrm{H}), 3.73(\mathrm{~s}, 1 \mathrm{H}), 5.19(\mathrm{~s}, 1 \mathrm{H}), 6.22(\mathrm{~s}, 1 \mathrm{H}), 6.70(\mathrm{~d}, J=8.7 \mathrm{~Hz}, 1 \mathrm{H}), 7.36(\mathrm{~d}, J=$ $2.1 \mathrm{~Hz}, 1 \mathrm{H}), 7.39(\mathrm{~d}, J=2.1 \mathrm{~Hz}, 1 \mathrm{H}), 7.56(\mathrm{dd}, J=8.5,2.1 \mathrm{~Hz}, 1 \mathrm{H}), 7.61(\mathrm{~d}, J=2.1 \mathrm{~Hz}, 1$ $\mathrm{H}) ;{ }^{13} \mathrm{C}$-APT NMR $\left(75 \mathrm{MHz}, \mathrm{CDCl}_{3}\right) \delta-4.8(+),-4.4(+), 17.4(+), 18.0(-), 21.4(-), 21.7(+)$, $23.8(+), 25.4(+), 27.5(+), 29.7(-), 38.9(+), 45.3(+), 83.1(-), 85.6(-), 122.6(+), 124.3(+)$, 127.8 (2 C, -), $131.7(-), 135.8(-), 136.9(+), 138.1$ (2 C, +), $140.7(+), 151.7(-), 151.9(-)$; HRMS (FAB) calcd for $\mathrm{C}_{28} \mathrm{H}_{38} \mathrm{O}_{2} \mathrm{SiI}_{2}\left[\mathrm{M}^{+}\right]$688.0731, found 688.0754.

Monoterpenylmagnolol (1)

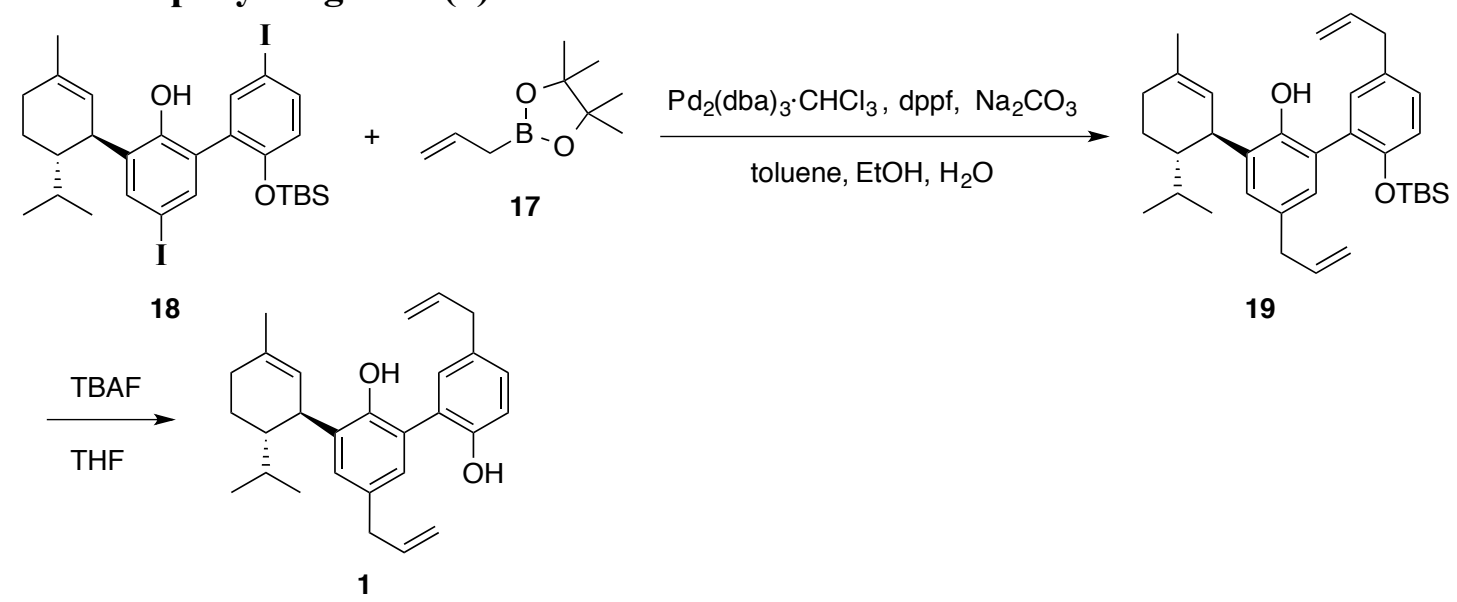

To a suspension of monophenol $18(18 \mathrm{mg}, 0.026 \mathrm{mmol})$, allylborate 17 (22 $\mathrm{mg}, 0.13$ $\mathrm{mmol})$, and $\mathrm{Na}_{2} \mathrm{CO}_{3}(17 \mathrm{mg}, 0.16 \mathrm{mmol})$ in toluene $(0.9 \mathrm{~mL}), \mathrm{EtOH}(1.2 \mathrm{~mL})$, and $\mathrm{H}_{2} \mathrm{O}(0.9$ $\mathrm{mL}$ ) were added $\mathrm{Pd}_{2}(\mathrm{dba})_{3} \cdot \mathrm{CHCl}_{3}(3 \mathrm{mg}, 0.0029 \mathrm{mmol})$ and dppf ( $\left.3 \mathrm{mg}, 0.0054 \mathrm{mmol}\right)$. The mixture was heated at $60{ }^{\circ} \mathrm{C}$ for $2 \mathrm{~h}$, cooled to $\mathrm{rt}$, and diluted with saturated $\mathrm{NH}_{4} \mathrm{Cl}$. The resulting mixture was extracted with EtOAc twice. The combined extracts were washed with brine, dried over $\mathrm{MgSO}_{4}$, and concentrated to give a residue, which was purified by chromatography on silica gel (hexane/EtOAc) to afford diallyl product 19 (10 mg, 74\% yield): $R_{\mathrm{f}} 0.76$ (hexane/EtOAc 6:1); ${ }^{1} \mathrm{H}$ NMR $\left(300 \mathrm{MHz}, \mathrm{CDCl}_{3}\right) \delta 0.05(\mathrm{~s}, 6 \mathrm{H}), 0.78(\mathrm{~s}, 9 \mathrm{H}), 0.89-$ 0.95 (m, $6 \mathrm{H}), 1.18-1.36(\mathrm{~m}, 2 \mathrm{H}), 1.55-1.67$ (m, $2 \mathrm{H}), 1.71(\mathrm{~s}, 3 \mathrm{H}), 1.94-2.08(\mathrm{~m}, 2 \mathrm{H}), 3.33$ $(\mathrm{d}, J=6.6 \mathrm{~Hz}, 2 \mathrm{H}), 3.37(\mathrm{~d}, J=6.9 \mathrm{~Hz}, 2 \mathrm{H}), 3.76-3.87(\mathrm{~m}, 1 \mathrm{H}), 4.97-5.14(\mathrm{~m}, 4 \mathrm{H}), 5.25(\mathrm{~s}$, $1 \mathrm{H}), 5.89-6.05$ (m, $2 \mathrm{H}), 6.27$ (br s, $1 \mathrm{H}), 6.83-6.96$ (m, $3 \mathrm{H}), 7.04-7.10$ (m, $1 \mathrm{H}), 7.10-7.16$ (m $1 \mathrm{H}$ ); HRMS (FAB) calcd for $\mathrm{C}_{34} \mathrm{H}_{48} \mathrm{O}_{2} \mathrm{Si}\left[\mathrm{M}^{+}\right]$516.3424, found 516.3410.

To an ice-cold solution of $19(9.8 \mathrm{mg}, 0.0193 \mathrm{mmol})$ in THF (3 mL) was added TBAF (1.0 $\mathrm{M}$ in THF, $0.020 \mathrm{~mL}, 0.020 \mathrm{mmol})$. The solution was stirred at $\mathrm{rt}$ for $1 \mathrm{~h}$ and diluted with saturated $\mathrm{NH}_{4} \mathrm{Cl}$. The resulting mixture was extracted with EtOAc three times. The combined extracts were washed with brine, dried over $\mathrm{MgSO}_{4}$, and concentrated to give a residue, which was purified by chromatography on silica gel (hexane/EtOAc) to afford monoterpenylmagnolol (1) (5.3 mg, 69\% yield): $[\alpha]_{\mathrm{D}}{ }^{20}-130\left(c 0.165, \mathrm{CHCl}_{3}\right)$ for $95 \%$ ee. $C f$. lit. $^{\mathrm{S} 4 \mathrm{a}}[\alpha]_{\mathrm{D}}{ }^{25}-129.1\left(c 0.004, \mathrm{CHCl}_{3}\right)$; lit. ${ }^{\mathrm{S} 4 \mathrm{~b}}[\alpha]_{\mathrm{D}}{ }^{25}-138.0\left(c 1.03, \mathrm{CHCl}_{3}\right) ;$ lit. $^{\mathrm{S} 4 \mathrm{c}}[\alpha]_{\mathrm{D}}{ }^{25}-135.5$ $\left(c 1.05, \mathrm{CHCl}_{3}\right)$; lit. ${ }^{\mathrm{S} 4 \mathrm{~d}}[\alpha]_{\mathrm{D}}{ }^{25}-146.0\left(c 1.18, \mathrm{CHCl}_{3}\right) ; R_{\mathrm{f}} 0.37$ (hexane/EtOAc 6:1); ${ }^{1} \mathrm{H}$ NMR $\left(400 \mathrm{MHz}, \mathrm{CDCl}_{3}\right) \delta 0.85(\mathrm{~d}, J=6.8 \mathrm{~Hz}, 3 \mathrm{H}), 0.89(\mathrm{~d}, J=6.8 \mathrm{~Hz}, 3 \mathrm{H}), 1.22-1.83(\mathrm{~m}, 4 \mathrm{H})$, $1.75(\mathrm{~s}, 3 \mathrm{H}), 2.06-2.19(\mathrm{~m}, 2 \mathrm{H}), 3.35(\mathrm{~d}, J=7.2 \mathrm{~Hz}, 2 \mathrm{H}), 3.37$ (d, $J=6.8 \mathrm{~Hz}, 2 \mathrm{H}), 3.45-$ $3.53(\mathrm{~m}, 1 \mathrm{H}), 5.02-5.15(\mathrm{~m}, 4 \mathrm{H}), 5.50(\mathrm{~s}, 1 \mathrm{H}), 5.90-5.65(\mathrm{~m}, 2 \mathrm{H}), 5.8-6.2(\mathrm{br} \mathrm{s}, 2 \mathrm{H})$ (exchanged with $\left.\mathrm{D}_{2} \mathrm{O}\right), 6.93$ (br s, $\left.1 \mathrm{H}\right), 6.97(\mathrm{~d}, J=8.0 \mathrm{~Hz}, 1 \mathrm{H}), 6.98$ (br s, $\left.1 \mathrm{H}\right), 7.09$ (br s, 1 $\mathrm{H}), 7.11(\mathrm{~d}, J=8.0 \mathrm{~Hz}, 1 \mathrm{H}) ;{ }^{13} \mathrm{C}-\mathrm{APT}\left(75 \mathrm{MHz}, \mathrm{CDCl}_{3}\right) \delta 16.5(+), 21.7(-), 21.7(+), 23.8$ 
$(+), 27.5(+), 30.4(-), 39.5(-), 42.6(+), 44.3(+), 115.69(-), 115.75(-), 117.2(+), 124.1(+)$, $125.1(-), 125.3(-), 129.6(+), 129.8(+), 130.9(+), 131.0(+), 131.2(+), 132.5(-), 132.7(-)$, $137.7(+), 137.8(+), 139.1(-), 149.2(-), 151.7(-)$; HRMS (FAB) calcd for $\mathrm{C}_{28} \mathrm{H}_{34} \mathrm{O}_{2}\left[\mathrm{M}^{+}\right]$ 402.2559, found 402.2554. The ${ }^{1} \mathrm{H}$ and ${ }^{13} \mathrm{C}$ NMR were updated. ${ }^{\text {S4a-d }}$ The structure was supported by the ${ }^{13} \mathrm{C}-\mathrm{APT}$ NMR signals, especially those appeared at 129-133 and 137-140 regions.

\section{Synthesis of $o-, p$-Diiodophenols}

\section{4,6-Diiodo-2-isopropylphenol (20)}

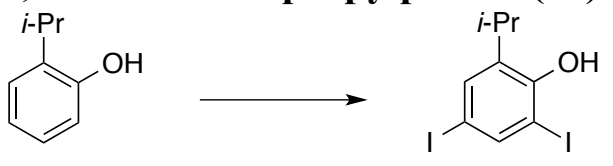

56

20

To an ice-cold solution of 2-isopropylphenol (56) (3.00 g, $22.0 \mathrm{mmol})$ and $\mathrm{NaOH}(2.19 \mathrm{~g}$, $54.8 \mathrm{mmol})$ in $\mathrm{MeOH}(44 \mathrm{~mL})$ were added $\mathrm{NaI}$ (16.5 g, $110 \mathrm{mmol})$ and $\mathrm{NaClO}$ (ca. 10\% purity, $114 \mathrm{~mL}$, ca. $176 \mathrm{mmol}$ ) dropwise. The mixture was stirred at $\mathrm{rt}$ for $30 \mathrm{~min}$ and diluted with aqueous $\mathrm{Na}_{2} \mathrm{~S}_{2} \mathrm{O}_{3}$ and $1 \mathrm{~N} \mathrm{HCl}$. The resulting mixture was extracted with EtOAc three times. The combined organic layers were washed with brine, dried over $\mathrm{MgSO}_{4}$, and concentrated to give a residue, which was purified by chromatography on silica gel (hexane/EtOAc) to afford 20 (6.68 g, 78\% yield): $R_{\mathrm{f}} 0.63$ (hexane/EtOAc 6:1); ${ }^{1} \mathrm{H}$ NMR (300 $\left.\mathrm{MHz} \mathrm{CDCl}_{3}\right) \delta 1.21(\mathrm{~d}, J=6.9 \mathrm{~Hz}, 6 \mathrm{H}), 3.24$ (sept., $\left.J=6.9 \mathrm{~Hz}, 1 \mathrm{H}\right), 5.31(\mathrm{~s}, 1 \mathrm{H}), 7.40(\mathrm{~d}, J$ $=2.1 \mathrm{~Hz}, 1 \mathrm{H}), 7.76(\mathrm{~d}, J=2.1 \mathrm{~Hz}, 1 \mathrm{H}) ;{ }^{13} \mathrm{C}$-APT NMR $\left(75 \mathrm{MHz}, \mathrm{CDCl}_{3}\right) \delta 22.3(+), 28.5$ $(+), 83.4(-), 87.9(-), 136.1(+), 137.8(-), 142.4(+), 152.0(-)$. The ${ }^{1}$ H NMR spectrum was identical with that reported. ${ }^{\text {S5 }}$

\section{4,6-Diiodo-2-methylphenol (24)}

According to the synthesis of 20, 2-methylphenol (103 mg, $0.952 \mathrm{mmol})$ was converted to 24 (265 mg, 77\% yield) by using $\mathrm{NaOH}$ (106 mg, $2.65 \mathrm{mmol}), \mathrm{NaI}$ (696 mg, $4.64 \mathrm{mmol}$ ), $\mathrm{NaClO}$ (ca. 10\% purity, $4.80 \mathrm{~mL}$, ca. $7.41 \mathrm{mmol})$, and $\mathrm{MeOH}(1.8 \mathrm{~mL}): R_{\mathrm{f}} 0.52$ (hexane/EtOAc 6:1); mp 63.8-64.2 ${ }^{\circ} \mathrm{C}$ (lit. $\left.{ }^{S 6} \mathrm{mp} 62-64{ }^{\circ} \mathrm{C}\right) ;{ }^{1} \mathrm{H}$ NMR $\left(300 \mathrm{MHz}, \mathrm{CDCl}_{3}\right) \delta$ $2.25(\mathrm{~s}, 3 \mathrm{H}), 5.30(\mathrm{~s}, 1 \mathrm{H}), 7.38(\mathrm{br} \mathrm{s}, 1 \mathrm{H}), 7.75(\mathrm{~d}, J=1.8 \mathrm{~Hz}, 1 \mathrm{H}) ;{ }^{13} \mathrm{C}-\mathrm{APT}$ NMR $(75$ $\left.\mathrm{MHz}, \mathrm{CDCl}_{3}\right) \delta 17.0(+), 82.7(-), 86.9(-), 127.4(-), 140.0(+), 142.7(+), 153.1(-)$. The ${ }^{1} \mathrm{H}$ and ${ }^{13} \mathrm{C}$ NMR spectra were identical with those reported. ${ }^{\mathrm{S} 7}$

\section{2-(tert-Butyl)-4,6-diiodophenol (25)}

According to the synthesis of 20, 2-(tert-butyl)phenol (146 mg, $0.972 \mathrm{mmol})$ was converted to 25 (256 mg, 66\% yield) by using $\mathrm{NaOH}$ (103 mg, $2.58 \mathrm{mmol})$, NaI (898 mg, $5.99 \mathrm{mmol}$ ), $\mathrm{NaClO}$ (ca. 10\% purity, $4.50 \mathrm{~mL}$, ca. $6.95 \mathrm{mmol}$ ), and $\mathrm{MeOH}(2 \mathrm{~mL}): R_{\mathrm{f}} 0.69$ (hexane/EtOAc 6:1); mp 56.3-57.0 ${ }^{\circ} \mathrm{C}$ (lit. $\left.{ }^{\mathrm{S} 8} \mathrm{mp} 55.5-57.5{ }^{\circ} \mathrm{C}\right) ;{ }^{1} \mathrm{H} \mathrm{NMR}\left(300 \mathrm{MHz}, \mathrm{CDCl}_{3}\right) \delta 1.37$ (s, $\left.9 \mathrm{H}\right)$, $5.53(\mathrm{~s}, 1 \mathrm{H}), 7.47(\mathrm{~d}, J=2.1 \mathrm{~Hz}, 1 \mathrm{H}), 7.80(\mathrm{~d}, J=2.1 \mathrm{~Hz}, 1 \mathrm{H}) ;{ }^{13} \mathrm{C}-\mathrm{APT}$ NMR $(75 \mathrm{MHz}$, $\left.\mathrm{CDCl}_{3}\right) \delta 29.2(+), 35.7(-), 83.2(-), 90.1(-), 136.8(+), 139.3(-), 143.1(+), 152.8(-)$. The ${ }^{1} \mathrm{H}$ and ${ }^{13} \mathrm{C}$ NMR spectra were identical with those reported. ${ }^{\mathrm{S} 9}$

\section{2,4-Diiodophenol (26)}

According to the synthesis of 20, phenol $(100 \mathrm{mg}, 1.06 \mathrm{mmol})$ was subjected to iodination 
with $\mathrm{NaOH}$ (109 mg, $2.73 \mathrm{mmol}$ ), NaI (1.44 g, $9.61 \mathrm{mmol}$ ), $\mathrm{NaClO}$ (ca. 5\% purity, $8.20 \mathrm{~mL}$, $12.7 \mathrm{mmol}$ ), and $\mathrm{MeOH}(2.2 \mathrm{~mL})$ to afford 26 (186 mg, 51\% yield) and 2,4,6-triiodophenol (127 mg, 25\% yield) after chromatography on silica gel. 2,4-Diiodophenol (26): $R_{\mathrm{f}} 0.29$ (hexane/EtOAc 6:1); mp 72.2-72.6 ${ }^{\circ} \mathrm{C}$ (lit. $\left.{ }^{\mathrm{S} 10} \mathrm{mp} 72-73{ }^{\circ} \mathrm{C}\right) ;{ }^{1} \mathrm{H}$ NMR $\left(300 \mathrm{MHz}, \mathrm{CDCl}_{3}\right)$ $\delta 5.29(\mathrm{~s}, 1 \mathrm{H}), 6.76(\mathrm{~d}, J=8.4 \mathrm{~Hz}, 1 \mathrm{H}), 7.51(\mathrm{dd}, J=8.4,2.0 \mathrm{~Hz}, 1 \mathrm{H}), 7.93(\mathrm{~d}, J=2.0 \mathrm{~Hz}, 1$ $\mathrm{H}) ;{ }^{13} \mathrm{C}$-APT NMR $\left(75 \mathrm{MHz}, \mathrm{CDCl}_{3}\right) \delta 82.9(-), 87.1(-), 117.1(+), 139.0(+), 145.4(+)$, $154.9(-)$. The ${ }^{1} \mathrm{H}$ and ${ }^{13} \mathrm{C}$ NMR spectra of 26 were identical with those reported. ${ }^{\mathrm{S} 10} \mathrm{The}{ }^{1} \mathrm{H}$ and ${ }^{13} \mathrm{C}$ NMR spectra of 2,4,6-triiodophenol were identical with those purchased from a commercial source.

\section{4,6-Diiodo-2-isopropyl-1-methoxybenzene (27)}

To an ice-cold solution of 20 (398 $\mathrm{mg}, 1.03 \mathrm{mmol})$ and $\mathrm{NaH}(60 \%$ content, $54 \mathrm{mg}, 1.35$ $\mathrm{mmol})$ in DMF $(2.5 \mathrm{~mL})$ was added MeI $(0.0770 \mathrm{~mL}, 1.24 \mathrm{mmol})$ dropwise. The mixture was stirred at $\mathrm{rt}$ for $30 \mathrm{~min}$ and diluted with saturated $\mathrm{NH}_{4} \mathrm{Cl}$. The resulting mixture was extracted with $\mathrm{Et}_{2} \mathrm{O}$ three times. The combined organic layers were washed with brine, dried over $\mathrm{MgSO}_{4}$, and concentrated to give a residue, which was purified by chromatography on silica gel (hexane/EtOAc) to afford 27 (316 mg, 77\% yield): $R_{\mathrm{f}} 0.40$ (hexane $/ \mathrm{CH}_{2} \mathrm{Cl}_{2}$ 6:1); mp 58.0$58.7{ }^{\circ} \mathrm{C}$; ${ }^{1} \mathrm{H} \mathrm{NMR}\left(300 \mathrm{MHz}, \mathrm{CDCl}_{3}\right) \delta 1.20$ (d, $\left.J=6.9 \mathrm{~Hz}, 6 \mathrm{H}\right), 3.27$ (sept., $\left.J=6.9 \mathrm{~Hz}, 1 \mathrm{H}\right)$, $3.77(\mathrm{~s}, 3 \mathrm{H}), 7.49(\mathrm{~d}, J=2.1 \mathrm{~Hz}, 1 \mathrm{H}), 7.92(\mathrm{~d}, J=2.1 \mathrm{~Hz}, 1 \mathrm{H}) ;{ }^{13} \mathrm{C}-$ APT NMR $(75 \mathrm{MHz}$, $\left.\mathrm{CDCl}_{3}\right) \delta 23.7(+), 27.7(+), 61.7(+), 89.4(-), 93.5(-), 136.4(+), 144.4(+), 145.5(-), 157.0$ (-); HRMS (FAB) calcd for $\mathrm{C}_{10} \mathrm{H}_{12} \mathrm{OI}_{2}\left[\mathrm{M}^{+}\right]$401.8978, found 401.8971 .

\section{4,6-Diiodo-2-isopropyl-1-(methoxymethoxy)benzene (28)}

To an ice-cold solution of $\mathbf{2 0}(502 \mathrm{mg}, 1.29 \mathrm{mmol})$ and $\mathrm{NaH}(60 \%$ content, $129 \mathrm{mg}, 3.23$ $\mathrm{mmol})$ in THF $(1.7 \mathrm{~mL})$ was added $\mathrm{MOMCl}(0.195 \mathrm{~mL}, 2.59 \mathrm{mmol})$ dropwise. The mixture was stirred at $\mathrm{rt}$ for $3 \mathrm{~h}$ and diluted with $\mathrm{MeOH}$ and $\mathrm{H}_{2} \mathrm{O}$. The resulting mixture was extracted with $\mathrm{Et}_{2} \mathrm{O}$ three times. The combined organic layers were washed with brine, dried over $\mathrm{MgSO}_{4}$, and concentrated to give a residue, which was purified by chromatography on silica gel (hexane/EtOAc) to afford 28 (439 mg, 79\% yield): $R_{\mathrm{f}} 0.29$ (hexane $/ \mathrm{CH}_{2} \mathrm{Cl}_{2} 6: 1$ ); mp 78.6$79.3{ }^{\circ} \mathrm{C} ;{ }^{1} \mathrm{H} \mathrm{NMR}\left(300 \mathrm{MHz}, \mathrm{CDCl}_{3}\right) \delta 1.19$ (d, $\left.J=6.9 \mathrm{~Hz}, 6 \mathrm{H}\right), 3.35$ (sept., $\left.J=6.9 \mathrm{~Hz}, 1 \mathrm{H}\right)$, $3.64(\mathrm{~s}, 3 \mathrm{H}), 5.02(\mathrm{~s}, 2 \mathrm{H}), 7.50(\mathrm{~d}, J=1.8 \mathrm{~Hz}, 1 \mathrm{H}), 7.93(\mathrm{~d}, J=1.8 \mathrm{~Hz}, 1 \mathrm{H}) ;{ }^{13} \mathrm{C}-\mathrm{APT}$ NMR $\left(75 \mathrm{MHz}, \mathrm{CDCl}_{3}\right) \delta 23.6(+), 27.5(+), 58.0(+), 89.8(-), 94.1(-), 100.5(-), 136.3(+), 144.5$ $(+), 146.0(-), 154.9(-)$; HRMS (EI) calcd for $\mathrm{C}_{11} \mathrm{H}_{14} \mathrm{O}_{2} \mathrm{I}_{2}\left[\mathrm{M}^{+}\right]$431.9083, found 431.9089 .

\section{tert-Butyl(4,6-diiodo-2-isopropylphenoxy)dimethylsilane (29)}

To an ice-cold solution of 20 (299 $\mathrm{mg}, 0.771 \mathrm{mmol})$ and $\mathrm{NaH}(60 \%$ content, $46 \mathrm{mg}, 1.15$ mmol) in THF ( $1 \mathrm{~mL})$ was added TBSCl $(234 \mathrm{mg}, 1.55 \mathrm{mmol})$. The mixture was stirred at $\mathrm{rt}$ overnight and diluted with $\mathrm{H}_{2} \mathrm{O}$. The resulting mixture was extracted with EtOAc three times. The combined organic layers were washed with brine, dried over $\mathrm{MgSO}_{4}$, and concentrated to give a residue, which was purified by chromatography on silica gel (hexane/EtOAc) to afford 29 (351 mg, 91\% yield): $R_{\mathrm{f}} 0.77$ (hexane/EtOAc 6:1); mp 51.3-51.9 ${ }^{\circ} \mathrm{C} ;{ }^{1} \mathrm{H} \mathrm{NMR}(300 \mathrm{MHz}$, $\left.\mathrm{CDCl}_{3}\right) \delta 0.32(\mathrm{~s}, 6 \mathrm{H}), 1.03(\mathrm{~s}, 9 \mathrm{H}), 1.13(\mathrm{~d}, J=6.9 \mathrm{~Hz}, 6 \mathrm{H}), 3.21$ (sept., $J=6.9 \mathrm{~Hz}, 1 \mathrm{H}$ ), $7.41(\mathrm{~d}, J=2.1 \mathrm{~Hz}, 1 \mathrm{H}), 7.89(\mathrm{~d}, J=2.1 \mathrm{~Hz}, 1 \mathrm{H}) ;{ }^{13} \mathrm{C}-\mathrm{APT}$ NMR $\left(75 \mathrm{MHz}, \mathrm{CDCl}_{3}\right) \delta-1.7$ $(+), 19.0(-), 23.1\left(_{(+)}, 26.4(+), 27.8(+), 85.7(-), 92.3(-), 135.5(+), 142.7(-), 144.6(+)\right.$, $152.8(-)$; HRMS (FAB) calcd for $\mathrm{C}_{15} \mathrm{H}_{24} \mathrm{OSiI}_{2}\left[\mathrm{M}^{+}\right]$501.9686, found 501.9689 . 


\section{$o$-Selective Deiodination of $o-, p$-Diiodophenols}

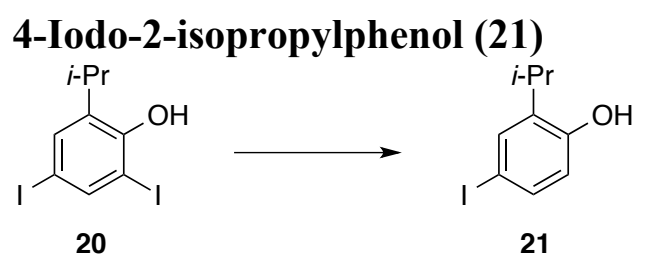

To an ice-cold solution of $20(57 \mathrm{mg}, 0.15 \mathrm{mmol})$ in $\mathrm{CH}_{2} \mathrm{Cl}_{2}(1.5 \mathrm{~mL})$ was added a toluene solution of Red-Al (3.07 M, $0.16 \mathrm{~mL}, 0.49 \mathrm{mmol})$ dropwise. The solution was stirred at $0{ }^{\circ} \mathrm{C}$ for $30 \mathrm{~min}$ and poured into $1 \mathrm{~N} \mathrm{HCl}$ carefully. The resulting mixture was extracted with $\mathrm{CH}_{2} \mathrm{Cl}_{2}$ twice. The combined organic layers were washed with brine, dried over $\mathrm{MgSO}_{4}$, and concentrated. The residue was purified by chromatography on silica gel (hexane/EtOAc) to afford 21 (34 mg, 89\% yield): $R_{\mathrm{f}} 0.33$ (hexane/EtOAc 6:1); ${ }^{1} \mathrm{H}$ NMR $\left(300 \mathrm{MHz}, \mathrm{CDCl}_{3}\right) \delta$ $1.23(\mathrm{~d}, J=6.9 \mathrm{~Hz}, 6 \mathrm{H}), 3.14$ (sept., $J=6.9 \mathrm{~Hz}, 1 \mathrm{H}), 4.82$ (br s, $1 \mathrm{H}), 6.52$ (d, $J=8.4 \mathrm{~Hz}, 1$ H), $7.34(\mathrm{dd}, J=8.4,2.4 \mathrm{~Hz}, 1 \mathrm{H}), 7.45(\mathrm{~d}, J=2.4 \mathrm{~Hz}, 1 \mathrm{H}) ;{ }^{13} \mathrm{C}-\mathrm{APT}$ NMR $(75 \mathrm{MHz}$, $\left.\mathrm{CDCl}_{3}\right) \delta 22.4(+), 27.0(+), 83.4(-), 117.6(+), 135.4(+), 137.4(-), 152.6(-)$. The ${ }^{1} \mathrm{H}$ and ${ }^{13} \mathrm{C}$ NMR spectra were identical with those reported. ${ }^{\text {S11 }}$

\section{4-Iodo-2-methylphenol (30)}

According to the deiodination of 20, an ice-cold solution of $24(50 \mathrm{mg}, 0.14 \mathrm{mmol})$ in $\mathrm{CH}_{2} \mathrm{Cl}_{2}(1.4 \mathrm{~mL})$ was added a toluene solution of Red-Al $(3.07 \mathrm{M}, 0.11 \mathrm{~mL}, 0.34 \mathrm{mmol})$, and the mixture was stirred at $0{ }^{\circ} \mathrm{C}$ for $30 \mathrm{~min}$ to afford $30(27 \mathrm{mg}, 83 \%$ yield $): R_{\mathrm{f}} 0.29$ (hexane/EtOAc 6:1); mp 67.7-68.1 ${ }^{\circ} \mathrm{C}$ (lit. $\left.{ }^{\mathrm{S} 12} \mathrm{mp} \mathrm{66.5-68}{ }^{\circ} \mathrm{C}\right) ;{ }^{1} \mathrm{H}$ NMR $\left(300 \mathrm{MHz}, \mathrm{CDCl}_{3}\right.$ ) $\delta 2.20(\mathrm{~s}, 3 \mathrm{H}), 4.76$ (br s, $1 \mathrm{H}), 6.55(\mathrm{~d}, J=8.4 \mathrm{~Hz}, 1 \mathrm{H}), 7.35$ (dd, $J=8.4,2.4 \mathrm{~Hz}, 1 \mathrm{H})$, 7.41-7.45 (m, 1 H); ${ }^{13} \mathrm{C}-\mathrm{APT} \operatorname{NMR}\left(75 \mathrm{MHz}, \mathrm{CDCl}_{3}\right) \delta 15.5(+), 82.7(-), 117.2(+), 126.8$ $(-), 135.9(+), 139.5(+), 153.8(-)$. The ${ }^{1} \mathrm{H}$ and ${ }^{13} \mathrm{C}$ NMR spectra were identical with those reported. ${ }^{\mathrm{S} 11}$

\section{2-(tert-Butyl)-4-iodophenol (31)}

According to the deiodination of 20, an ice-cold solution of $25(50 \mathrm{mg}, 0.12 \mathrm{mmol})$ in $\mathrm{CH}_{2} \mathrm{Cl}_{2}(1.3 \mathrm{~mL})$ was added a toluene solution of Red-Al $(3.07 \mathrm{M}, 0.10 \mathrm{~mL}, 0.307 \mathrm{mmol})$, and the solution was stirred at $0{ }^{\circ} \mathrm{C}$ for $2 \mathrm{~h}$ to afford 31 (29 $\mathrm{mg}, 84 \%$ yield): $R_{\mathrm{f}} 0.39$ (hexane/EtOAc 6:1); ${ }^{1} \mathrm{H}$ NMR (300 MHz, $\left.\mathrm{CDCl}_{3}\right) \delta 1.39(\mathrm{~s}, 9 \mathrm{H}), 4.80(\mathrm{~s}, 1 \mathrm{H}), 6.45$ (d, $J=$ $8.1 \mathrm{~Hz}, 1 \mathrm{H}), 7.35(\mathrm{dd}, J=8.1,2.1 \mathrm{~Hz}, 1 \mathrm{H}), 7.51(\mathrm{~d}, J=2.1 \mathrm{~Hz}, 1 \mathrm{H}) ;{ }^{13} \mathrm{C}-\mathrm{APT}$ NMR $(75$ $\left.\mathrm{MHz}, \mathrm{CDCl}_{3}\right) \delta 29.4(+), 34.7(-), 83.3(-), 118.8(+), 135.8(+), 136.1(+), 139.1(-), 154.2$ $(-)$. The ${ }^{1} \mathrm{H}$ NMR spectrum was updated and the ${ }^{13} \mathrm{C}$ NMR spectrum was identical with that reported. $^{\mathrm{S} 13}$

\section{4-Iodophenol (32)}

According to the deiodination of 20, an ice-cold solution of $\mathbf{2 6}(73 \mathrm{mg}, 0.21 \mathrm{mmol})$ in $\mathrm{CH}_{2} \mathrm{Cl}_{2}(2.2 \mathrm{~mL})$ was added a toluene solution of Red-Al $(3.07 \mathrm{M}, 0.18 \mathrm{~mL}, 0.55 \mathrm{mmol})$, and the solution was stirred at $0{ }^{\circ} \mathrm{C}$ for $5 \mathrm{~h}$ to afford 32 (39 mg, 84\% yield): $R_{\mathrm{f}} 0.26$ (hexane/EtOAc 6:1); ${ }^{1} \mathrm{H}$ NMR (300 MHz, $\mathrm{CDCl}_{3}$ ) $\delta 4.81$ (br s, $\left.1 \mathrm{H}\right), 6.63$ (dm, $J=9.0 \mathrm{~Hz}, 1$ $\mathrm{H}), 7.52(\mathrm{dm}, J=9.0 \mathrm{~Hz}, 1 \mathrm{H}) ;{ }^{13} \mathrm{C}$-APT NMR $\left(75 \mathrm{MHz}, \mathrm{CDCl}_{3}\right) \delta 82.9(-), 117.9(+), 138.5$ $(+), 155.2(-)$. The ${ }^{1} \mathrm{H}$ and ${ }^{13} \mathrm{C}$ NMR spectra were identical with those reported. ${ }^{\mathrm{S} 4}$ 


\section{4-Iodo-2-isopropyl-1-methoxybenzene (33)}

According to the deiodination of 20, an ice-cold solution of $27(47 \mathrm{mg}, 0.12 \mathrm{mmol})$ in $\mathrm{CH}_{2} \mathrm{Cl}_{2}(1.2 \mathrm{~mL})$ was added a toluene solution of Red-Al (3.07 M, $\left.0.06 \mathrm{~mL}, 0.18 \mathrm{mmol}\right)$, and the solution was stirred at $0{ }^{\circ} \mathrm{C}$ for $30 \mathrm{~min}$ to afford $33\left(26 \mathrm{mg}, 81 \%\right.$ yield): $R_{\mathrm{f}} 0.63$ (hexane/ $\mathrm{CH}_{2} \mathrm{Cl}_{2} 3: 1$ ); ${ }^{1} \mathrm{H}$ NMR $\left(300 \mathrm{MHz}, \mathrm{CDCl}_{3}\right.$ ) $\delta 1.18$ (d, $\left.J=6.9 \mathrm{~Hz}, 6 \mathrm{H}\right), 3.24$ (sept., $J=$ $6.9 \mathrm{~Hz}, 1 \mathrm{H}), 3.79(\mathrm{~s}, 3 \mathrm{H}), 6.60(\mathrm{dm}, J=9.0 \mathrm{~Hz}, 1 \mathrm{H}), 7.439(\mathrm{dd}, J=9.0,2.1 \mathrm{~Hz}, 1 \mathrm{H}), 7.443$ $(\mathrm{d}, J=2.1 \mathrm{~Hz}, 1 \mathrm{H}) ;{ }^{13} \mathrm{C}-$ APT NMR $\left(75 \mathrm{MHz}, \mathrm{CDCl}_{3}\right) \delta 22.6(+), 26.7(+), 55.5(+), 83.4(-)$, $112.7(+), 135.0(+), 135.3(+), 139.9(-), 156.7(-)$; HRMS (EI) calcd for $\mathrm{C}_{10} \mathrm{H}_{13} \mathrm{IO}\left[\mathrm{M}^{+}\right]$ 276.0011 , found 276.0015 .

\section{4-Iodo-2-isopropyl-1-(methoxymethoxy)benzene (34)}

According to the general procedure, an ice-cold solution of $\mathbf{2 8}(49 \mathrm{mg}, 0.11 \mathrm{mmol})$ in $\mathrm{CH}_{2} \mathrm{Cl}_{2}(1.2 \mathrm{~mL})$ was added a toluene solution of Red-Al (3.07 M, $\left.0.06 \mathrm{~mL}, 0.18 \mathrm{mmol}\right)$, and the solution was stirred at $0{ }^{\circ} \mathrm{C}$ for $30 \mathrm{~min}$ to afford $34\left(30 \mathrm{mg}, 86 \%\right.$ yield): $R_{\mathrm{f}} 0.37$ (hexane/ $\mathrm{CH}_{2} \mathrm{Cl}_{2} 3: 1$ ); ${ }^{1} \mathrm{H}$ NMR $\left(300 \mathrm{MHz}, \mathrm{CDCl}_{3}\right) \delta 1.20$ (d, $J=6.9 \mathrm{~Hz}, 6 \mathrm{H}$ ), 3.27 (sept., $J=$ $6.9 \mathrm{~Hz}, 1 \mathrm{H}), 3.47(\mathrm{~s}, 3 \mathrm{H}), 5.18(\mathrm{~s}, 2 \mathrm{H}), 6.83(\mathrm{~d}, J=8.7 \mathrm{~Hz}, 1 \mathrm{H}), 7.42(\mathrm{dd}, J=8.7,2.4 \mathrm{~Hz}, 1$ $\mathrm{H}), 7.48(\mathrm{~d}, J=2.4 \mathrm{~Hz}, 1 \mathrm{H}) ;{ }^{13} \mathrm{C}-\mathrm{APT}$ NMR $\left(75 \mathrm{MHz}, \mathrm{CDCl}_{3}\right) \delta 22.7(+), 27.0(+), 56.1(+)$, $85.0(-), 94.4(-), 116.2(+), 135.3(+), 135.4(+), 140.4(-), 154.3(-)$. The ${ }^{1} \mathrm{H}$ and ${ }^{13} \mathrm{C}$ NMR spectra were identical with those reported. ${ }^{\mathrm{S} 15}$

\section{tert-Butyl(4-iodo-2-isopropylphenoxy)dimethylsilane (35)}

According to the general procedure, an ice-cold solution of $29(46 \mathrm{mg}, 0.092 \mathrm{mmol})$ in $\mathrm{CH}_{2} \mathrm{Cl}_{2}(0.9 \mathrm{~mL})$ was added a toluene solution of Red-Al (3.07 M, $\left.0.05 \mathrm{~mL}, 0.15 \mathrm{mmol}\right)$, and the solution was stirred at $0{ }^{\circ} \mathrm{C}$ for $30 \mathrm{~min}$ to afford $35\left(25 \mathrm{mg}, 73 \%\right.$ yield): $R_{\mathrm{f}} 0.63$ (hexane only); ${ }^{1} \mathrm{H}$ NMR (300 MHz, $\left.\mathrm{CDCl}_{3}\right) \delta 0.22(\mathrm{~s}, 6 \mathrm{H}), 1.00$ (s, $\left.9 \mathrm{H}\right), 1.17$ (d, $\left.J=6.9 \mathrm{~Hz}, 6 \mathrm{H}\right)$, 3.22 (sept., $J=6.9 \mathrm{~Hz}, 1 \mathrm{H}$ ), 6.53 (d, $J=8.4 \mathrm{~Hz}, 1 \mathrm{H}), 7.32$ (dd, $J=8.4,2.4 \mathrm{~Hz}, 1 \mathrm{H}), 7.45$ (d, $J=2.4 \mathrm{~Hz}, 1 \mathrm{H}) ;{ }^{13} \mathrm{C}-$ APT NMR $\left(75 \mathrm{MHz}, \mathrm{CDCl}_{3}\right) \delta-4.1(+), 18.3(-), 22.7(+), 25.8(+)$, $26.7(+), 84.0(-), 120.6(+), 135.2(+), 135.4(+), 142.1(-), 152.8(-)$. The ${ }^{1}$ H NMR spectrum was identical with that reported, ${ }^{\mathrm{S} 16}$ whereas the ${ }^{13} \mathrm{C}$ NMR spectrum in the literature ${ }^{\mathrm{S} 16}$ was revised. 


\section{References}

(S1) (a) Stiles, M. J. Org. Chem. 1994, 59, 5381. (b) Tabaei, S.-M. H.; Pittman, C. U., Jr.; Mead, K. T. J. Org. Chem. 1992, 57, 6669.

(S2) Houjeiry, T. I.; Poe, S. L.; McQuade, D. T. Org. Lett. 2012, 14, 4394.

(S3) (a) Zhang, H.-C.; Huang, W.-S.; Pu, L. J. Org. Chem. 2001, 66, 481. (b) Frank, D. J.; Franzke, A.; Pfaltz, A. Chem. Eur. J. 2013, 19, 2405.

(S4) (a) Agharahimi, M. R.; LeBel, N. A. J. Org. Chem. 1995, 60, 1856. (b) Konoshima, T.; Kozuka, M.; Tokuda, H.; Nishino, H.; Iwashima, A.; Haruna, M.; Ito, K.; Tanabe, M. J. Nat. Prod. 1991, 54, 816. (c) Youn, U. J.; Lee, I. S.; Chen, Q. C.; Na, M.-K.; Jung, H. J.; Lee, S. M.; Choi, J. S.; Woo, M. H.; Bae, K.-H.; Min, B.-S. Nat. Prod. Sci. 2011, 17, 95. (d) Yahara, S.; Nishiyori, T.; Kohda, A.; Nohara, T.; Nishioka, I, Chem. Pharm. Bull. 1991, 39, 2024.

(S5) Jendralla, H.; Wess, G.; Bartmann, W.; Beck, G. Eur. Pat. 1989, EP 341681 A2 19891115.

(S6) Kajigaeshi, S.; Kakinami, T.; Yamasaki, H.; Fujisaki, S.; Kondo, M.; Okamoto, T. Chem. Lett. 1987, 2109.

(S7) Kometani, T.; Watt, D. S.; Ji, T.; Fitz, T. J. Org. Chem. 1985, 50, 5384.

(S8) Tashiro, M.; Fukata, G. J. Org. Chem. 1977, 42, 428.

(S9) Anderson, S.; Taylor, P. N.; Verschoor, G. L. B. Chem. Eur. J. 2004, 10, 518.

(S10) Petrone, D. A.; Lischka, M.; Lautens, M. Angew. Chem. Int. Ed. 2013, 52, 10635.

(S11) Peters, M.; Trobe, M.; Tan, H.; Kleineweischede, R.; Breinbauer, R. Chem. Eur. J. 2013, 19, 2442.

(S12) Pearson, D. E.; Frazer, M. G.; Frazer, V. S.; Washburn, L. C. Synthesis 1976, 621.

(S13) Bombis, C.; Weigelt, S.; Knudsen, M. M.; Nørgaard, M.; Busse, C.; Lægsgaard, E.; Besenbacher, F.; Gothelf, K. V.; Linderoth, T. R. ACS Nano 2010, 4, 297.

(S14) Zhou, C.-Y.; Li, J.; Peddibhotla, S.; Romo, D. Org. Lett. 2010, 12, 2104.

(S15) Placzek, A. T.; Scanlan, T. S. Tetrahedron 2015, 71, 5946.

(S16) Pérez, C. R.; López-Pérez, D.; Chmielewski, J.; Lipton, M. Chem. Biol. Drug Des. 2012, 79, 260. 


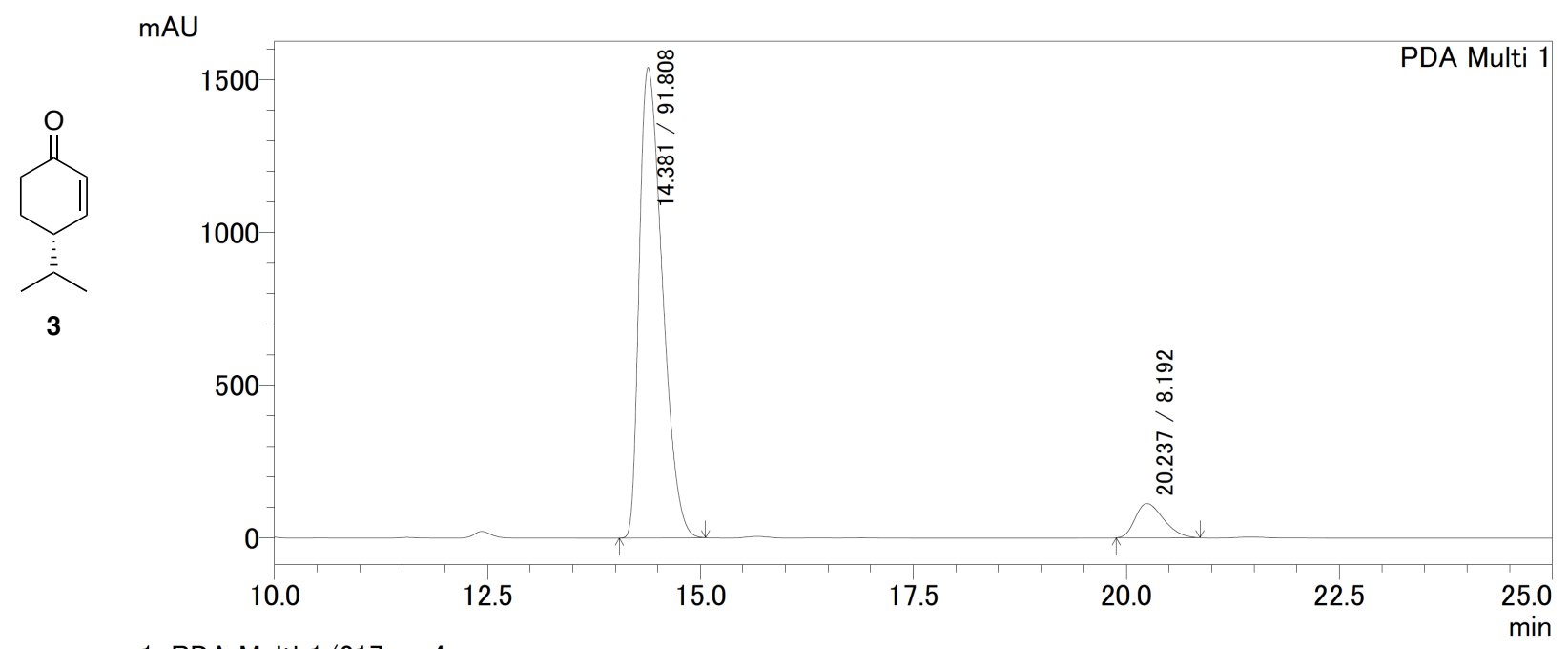

1 PDA Multi $1 / 217 \mathrm{~nm} 4 \mathrm{~nm}$

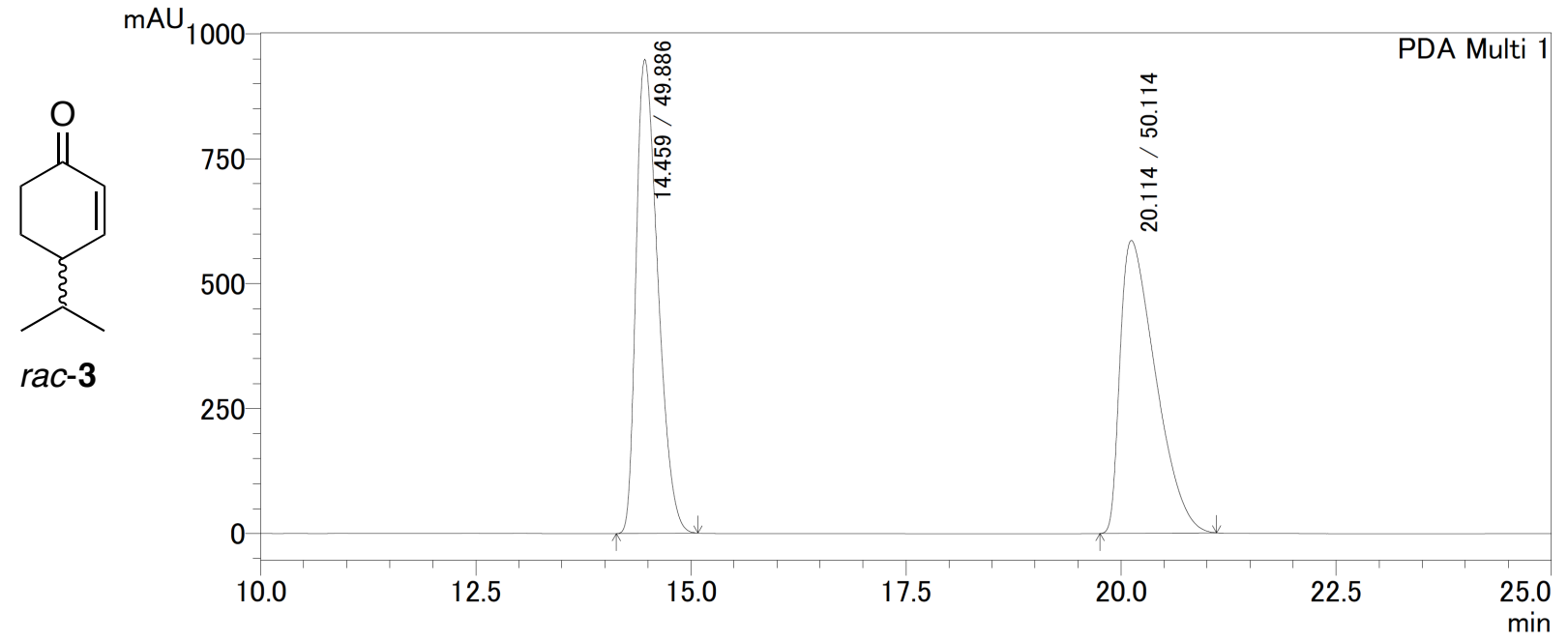

1 PDA Multi 1/217nm 4nm 


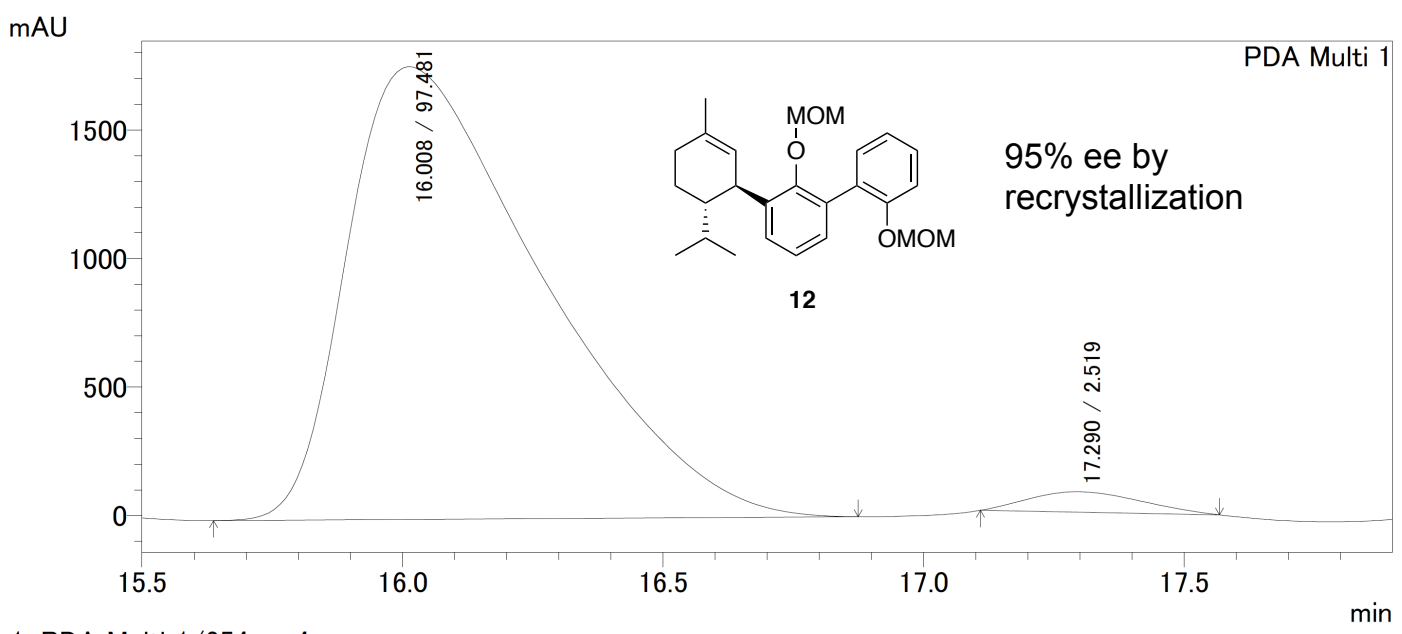

1 PDA Multi 1/254nm 4nm

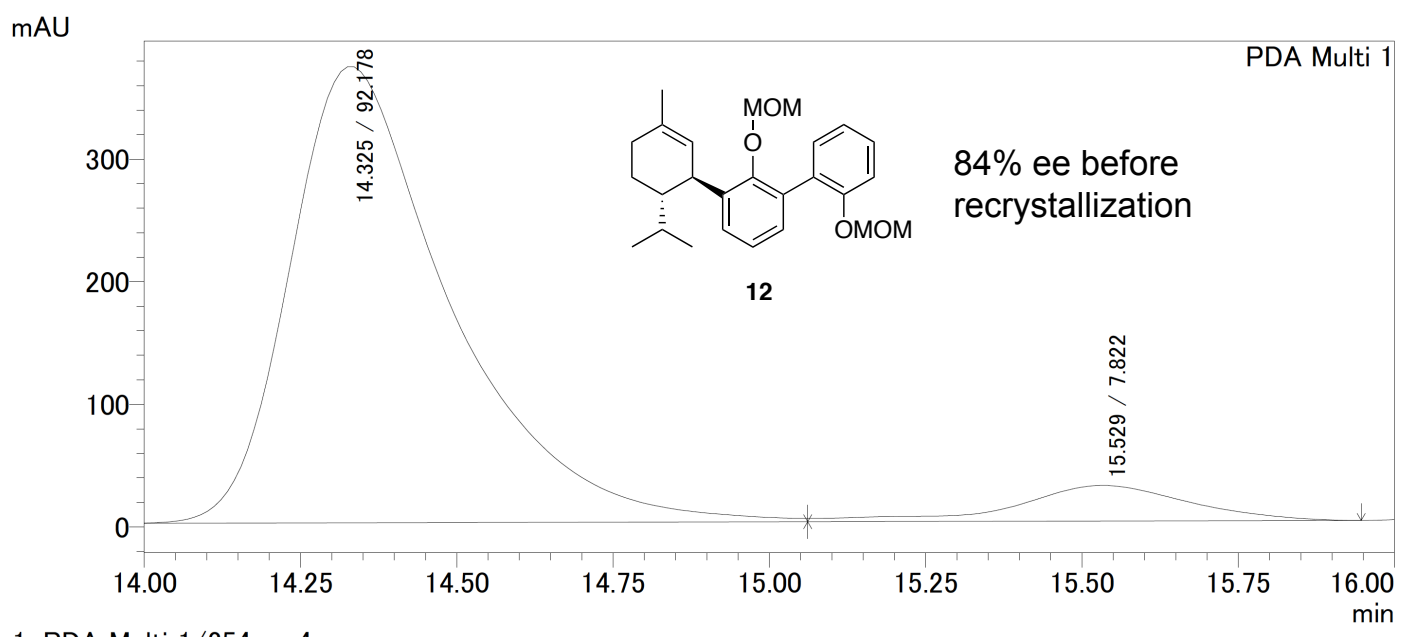

1 PDA Multi $1 / 254 \mathrm{~nm} 4 \mathrm{~nm}$

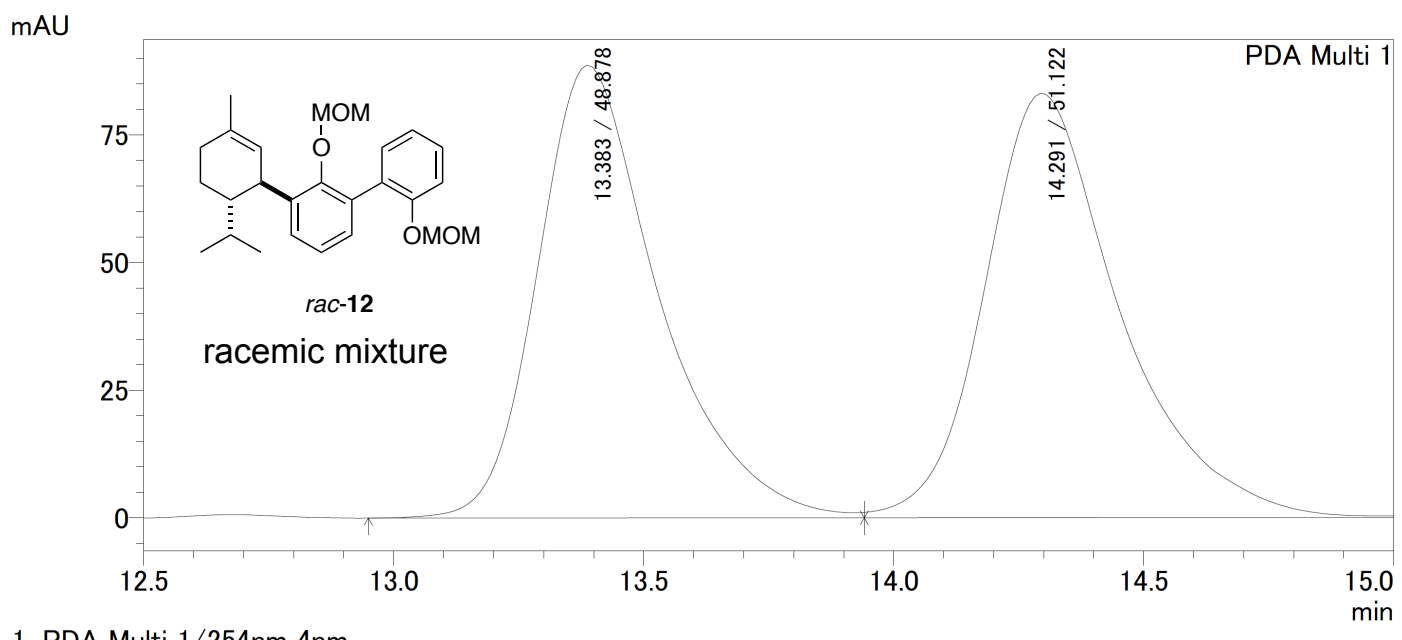

1 PDA Multi 1/254nm 4nm 

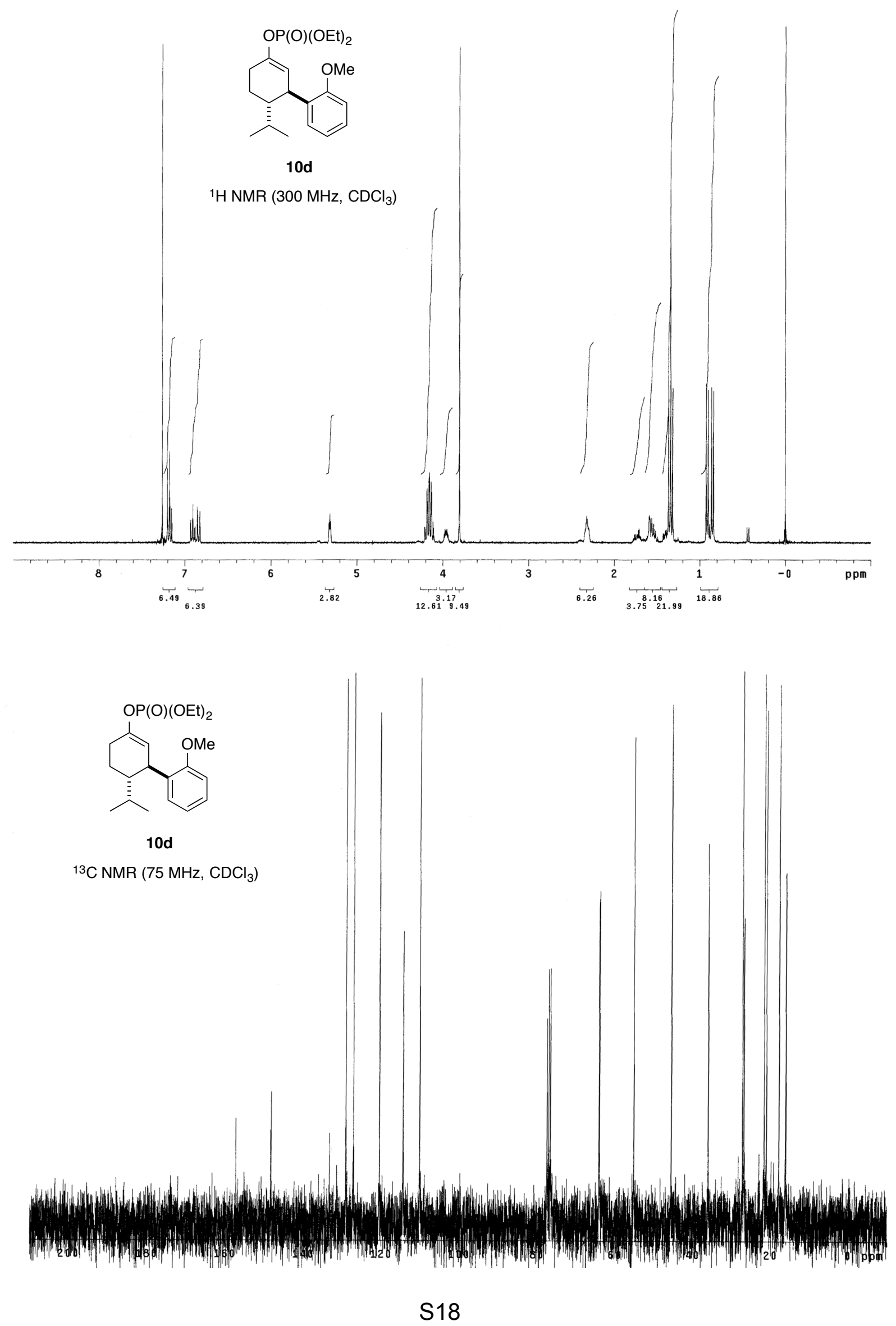


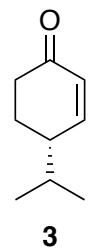

${ }^{1} \mathrm{H}$ NMR $\left(300 \mathrm{MHz}, \mathrm{CDCl}_{3}\right)$

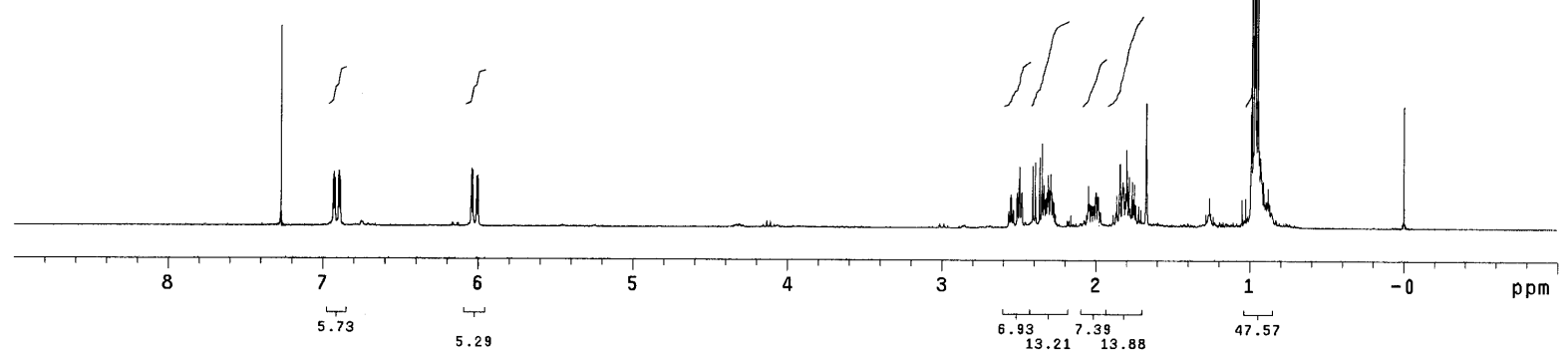<smiles>CC(C)C1C=CC(=O)CC1</smiles>

${ }^{13} \mathrm{C}$ NMR $\left(75 \mathrm{MHz}, \mathrm{CDCl}_{3}\right.$ )

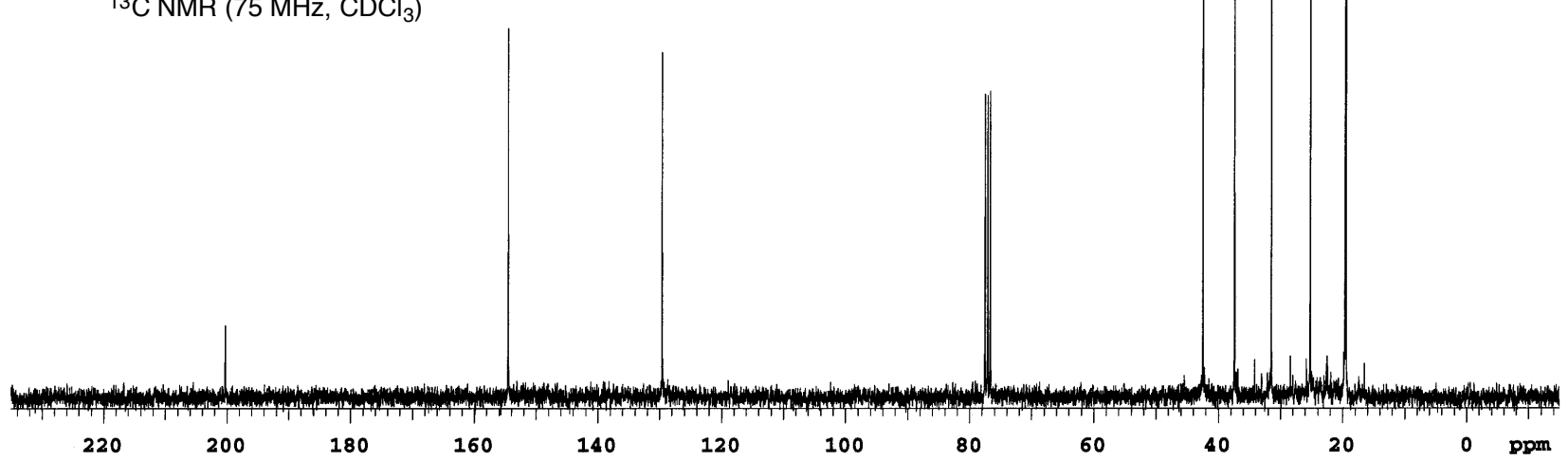



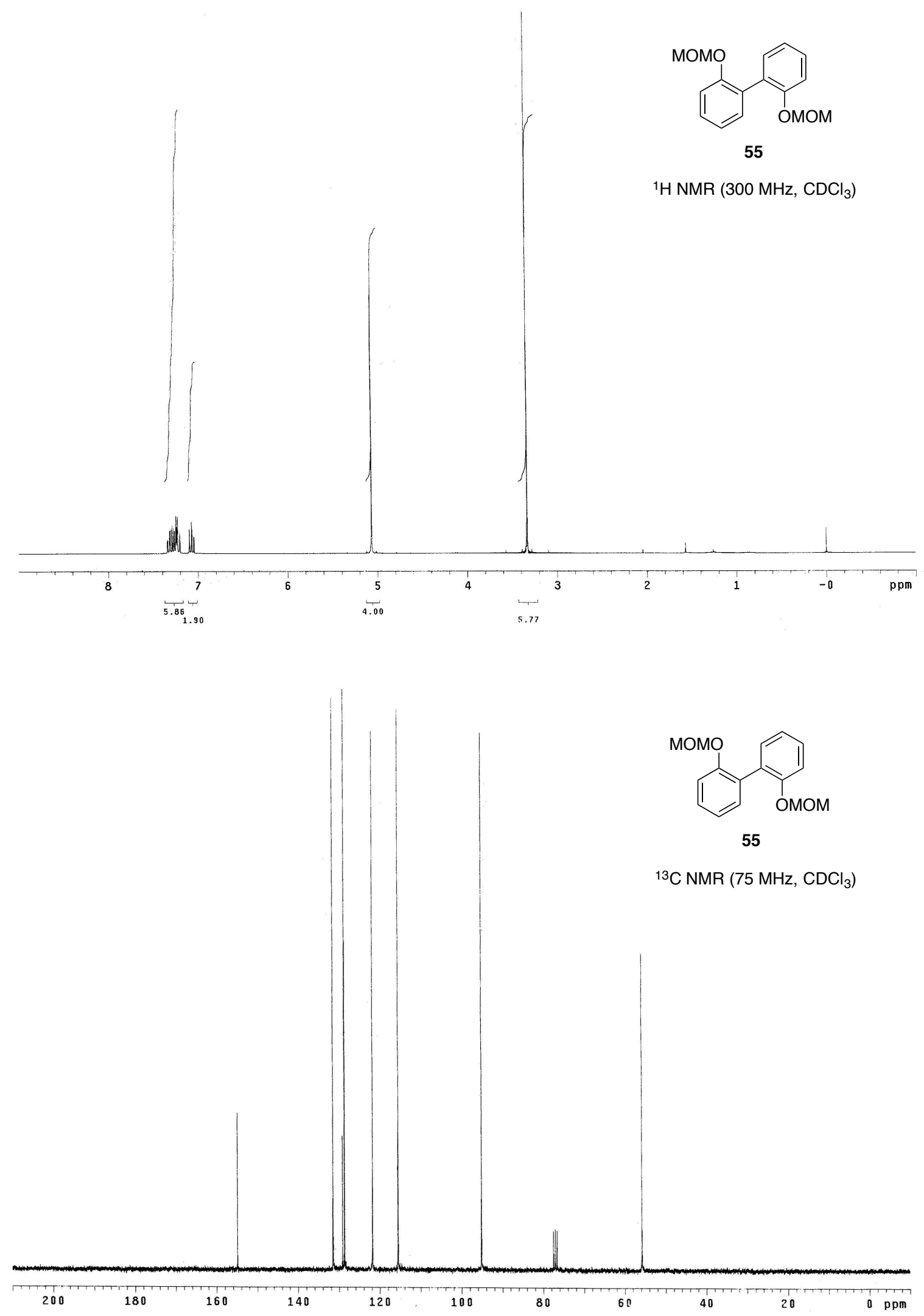

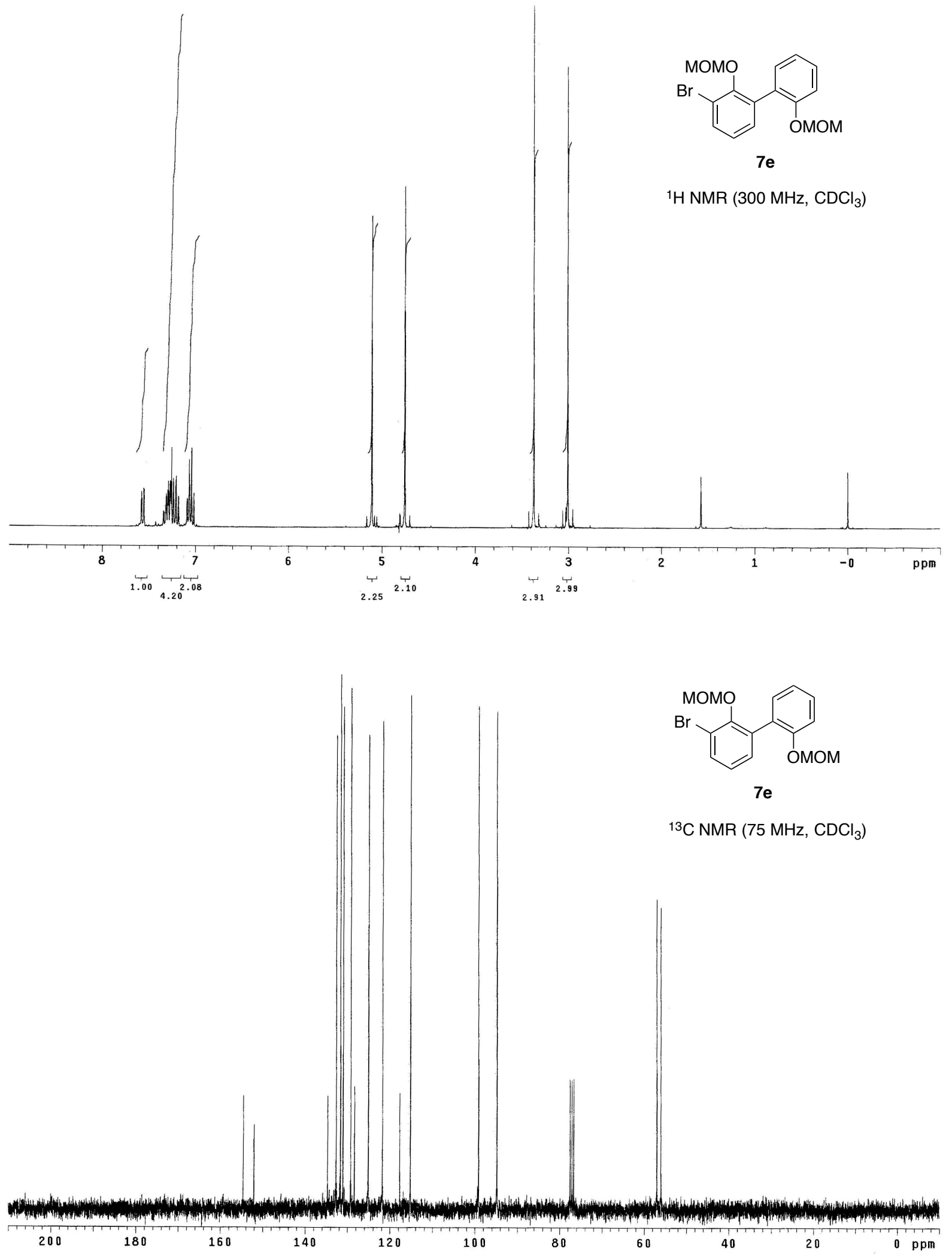


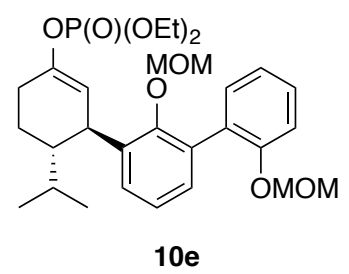

${ }^{1} \mathrm{H}$ NMR (400 MHz, $\mathrm{CDCl}_{3}$ )
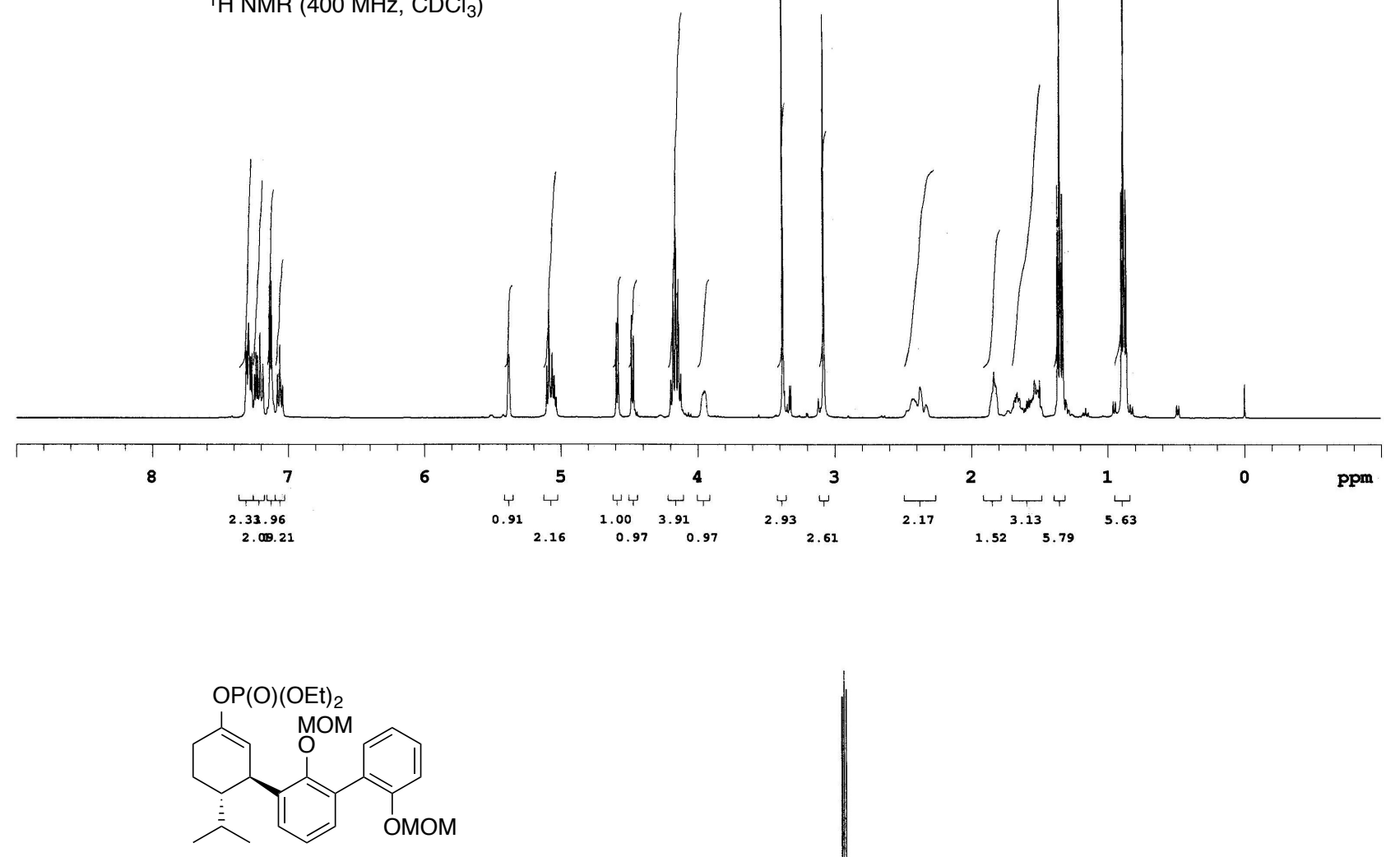

$10 \mathrm{e}$

${ }^{13} \mathrm{C}$ NMR $\left(100 \mathrm{MHz}, \mathrm{CDCl}_{3}\right)$

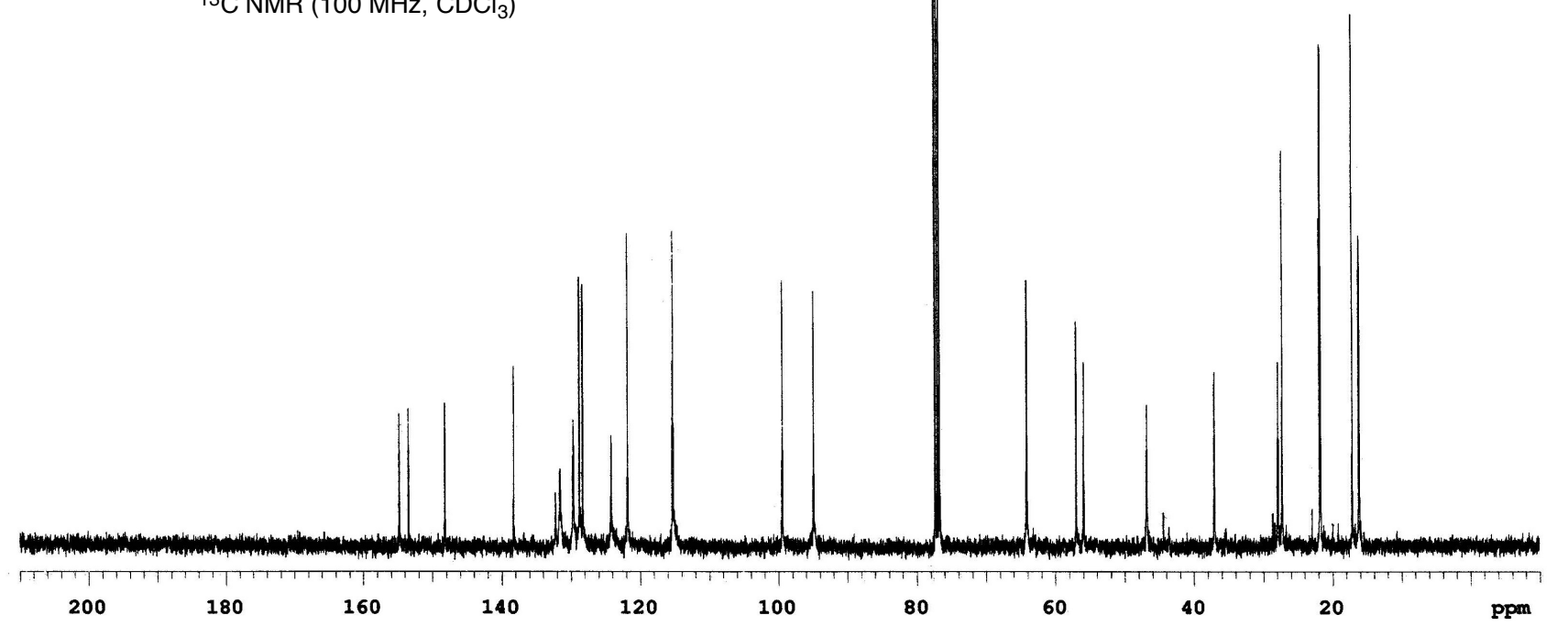




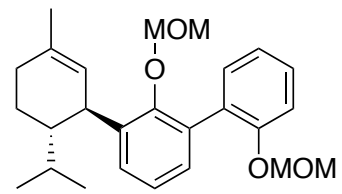

12

${ }^{1} \mathrm{H}$ NMR $\left(400 \mathrm{MHz}, \mathrm{CDCl}_{3}\right)$
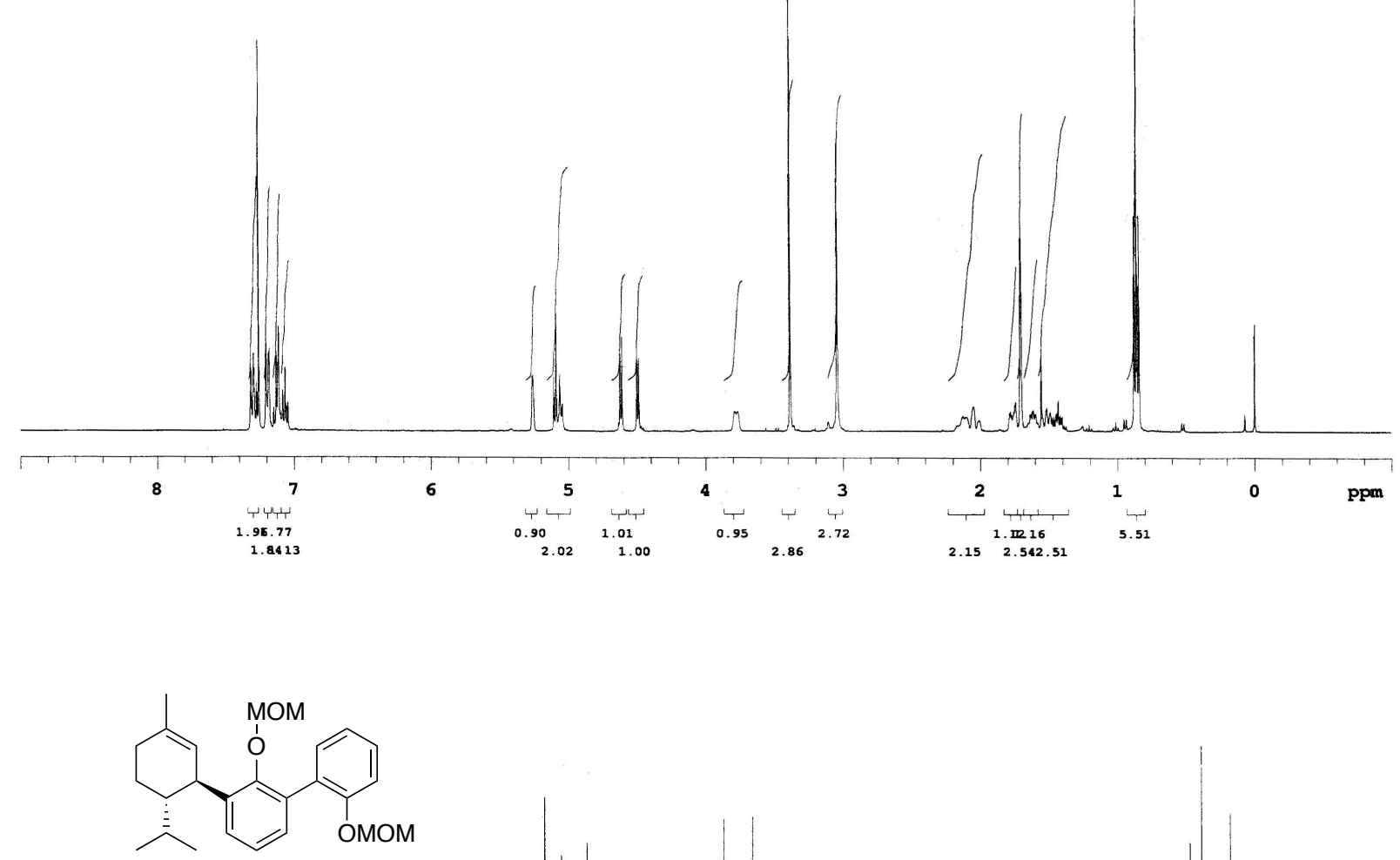

12

${ }^{13} \mathrm{C} \mathrm{NMR}\left(75 \mathrm{MHz}, \mathrm{CDCl}_{3}\right)$

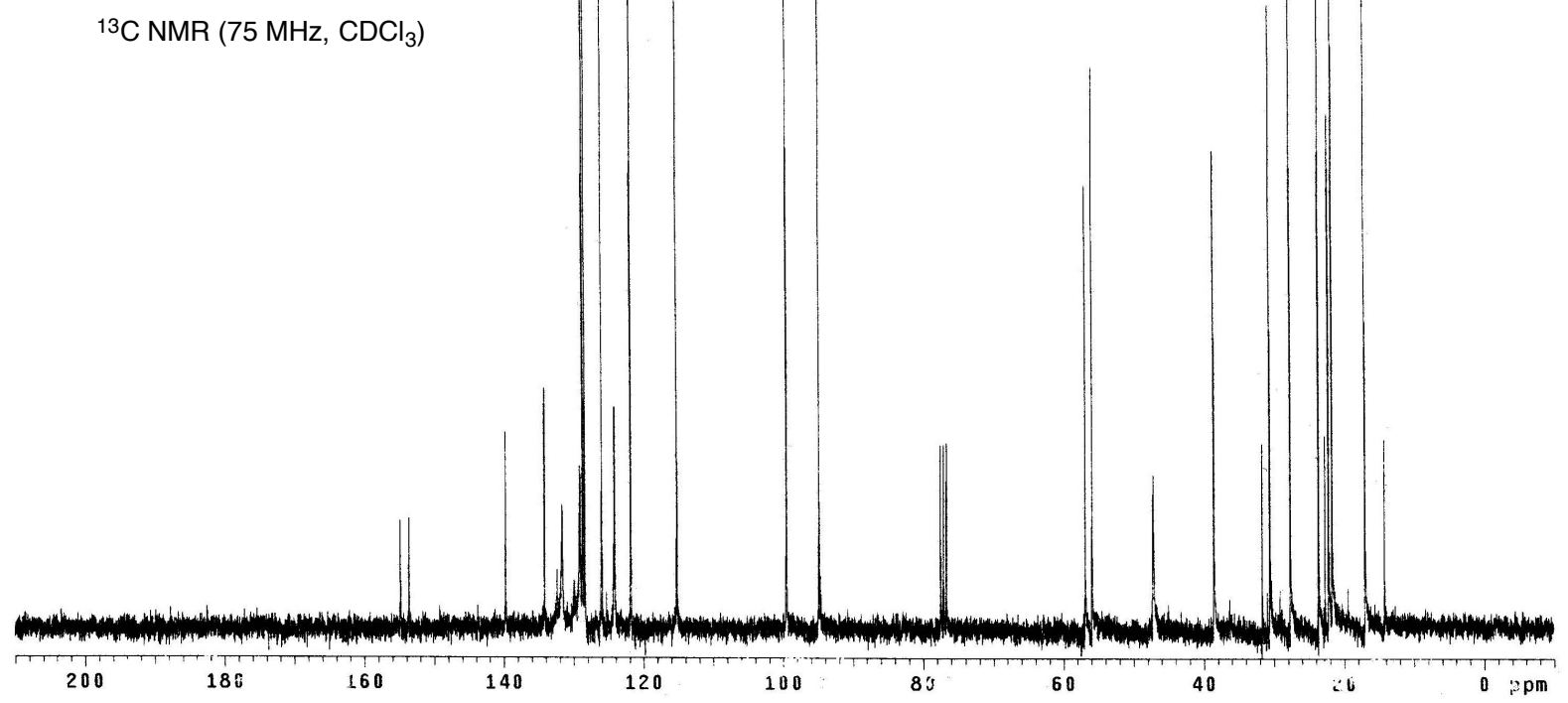



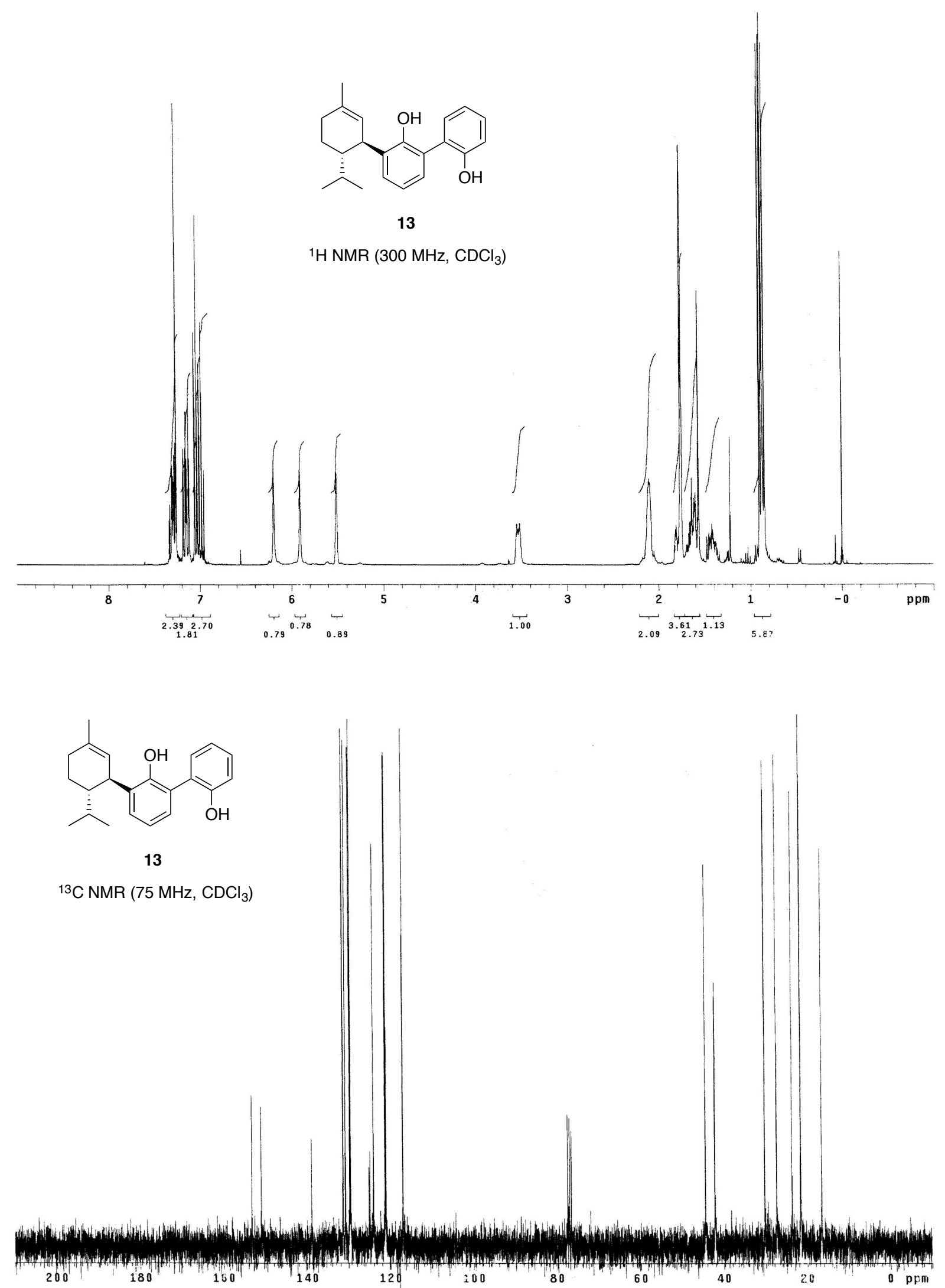

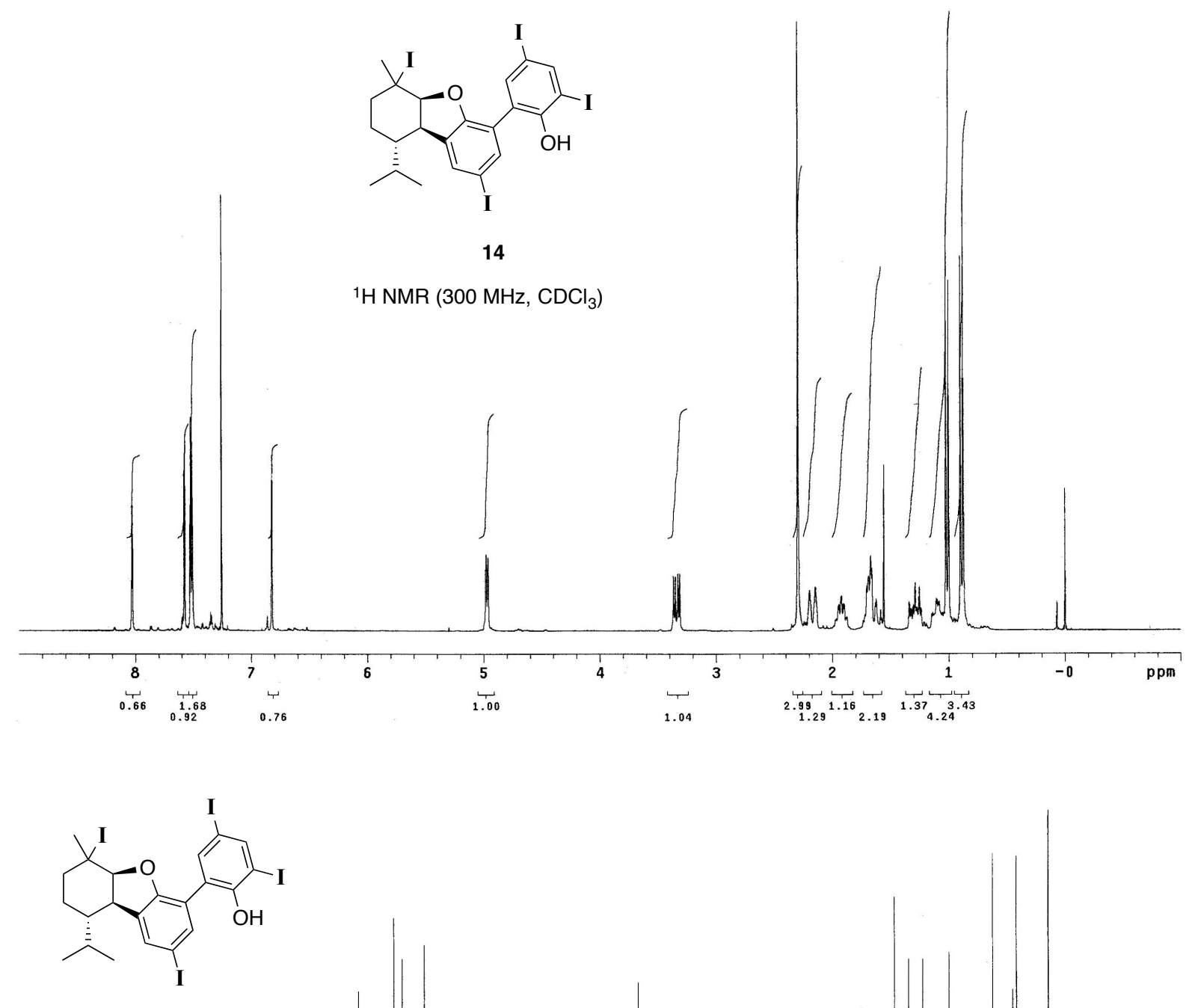

14

${ }^{13} \mathrm{C}$ NMR $\left(75 \mathrm{MHz}, \mathrm{CDCl}_{3}\right)$

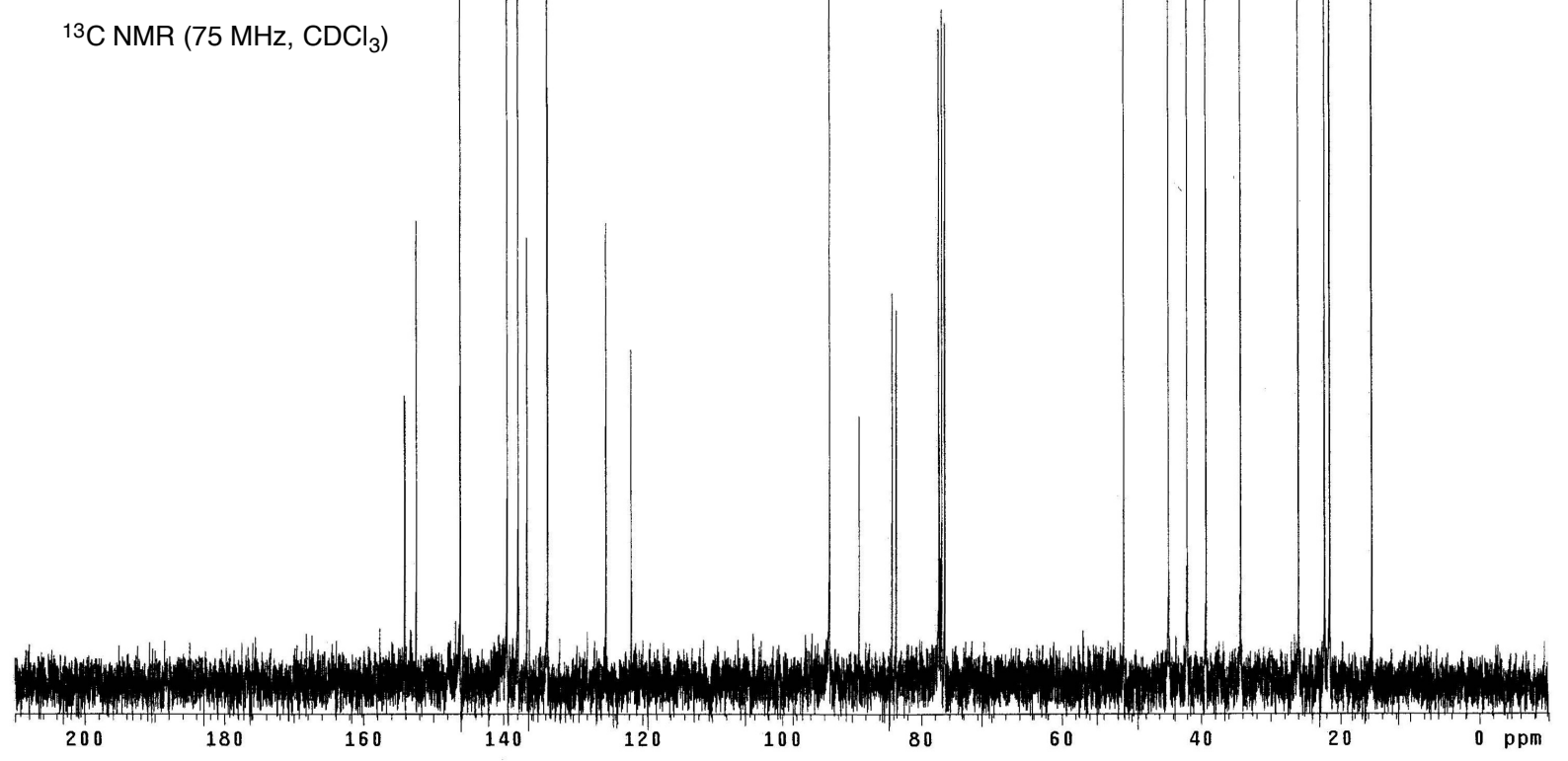



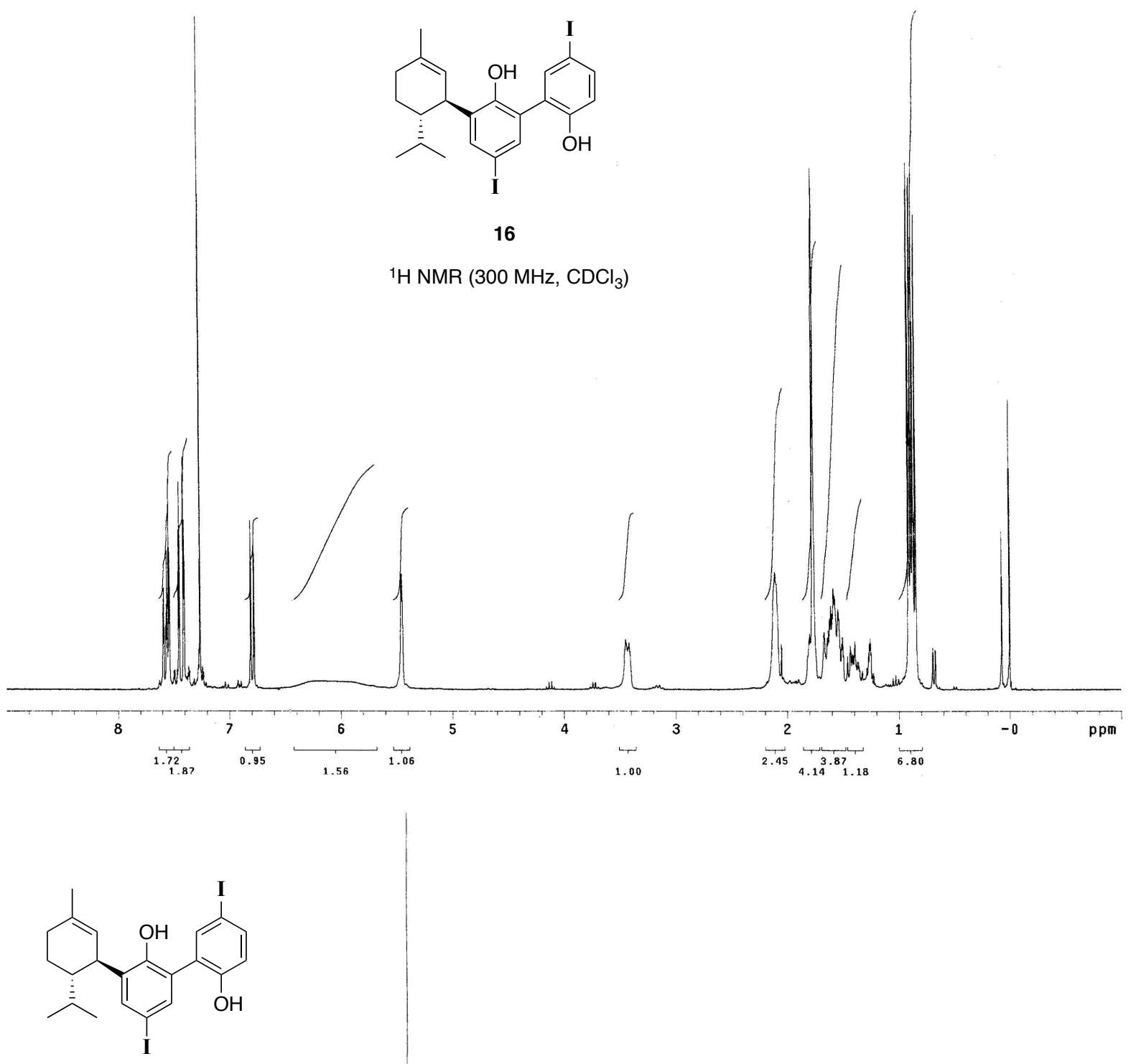

16

${ }^{13} \mathrm{C} \mathrm{NMR}\left(75 \mathrm{MHz}, \mathrm{CDCl}_{3}\right)$

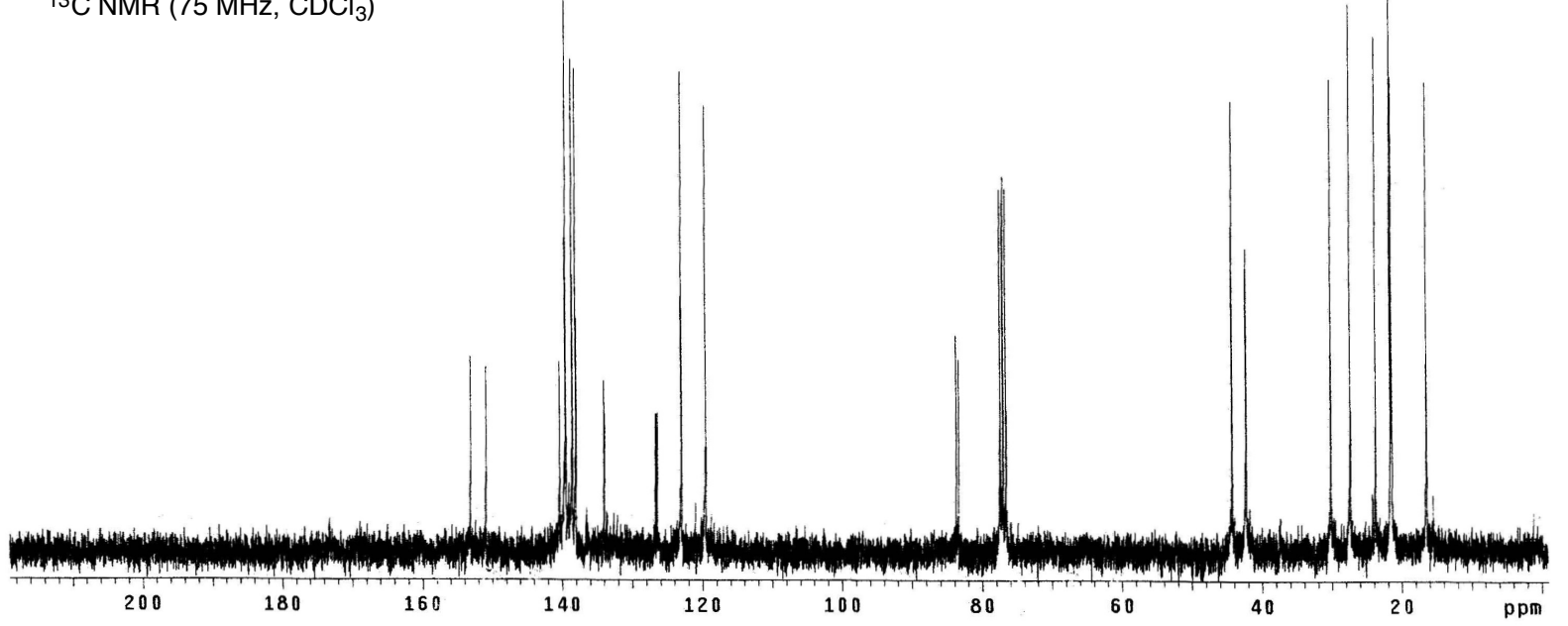




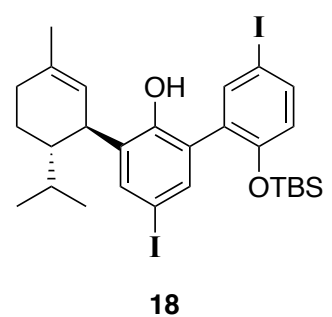

${ }^{1} \mathrm{H}$ NMR $\left(300 \mathrm{MHz}, \mathrm{CDCl}_{3}\right)$
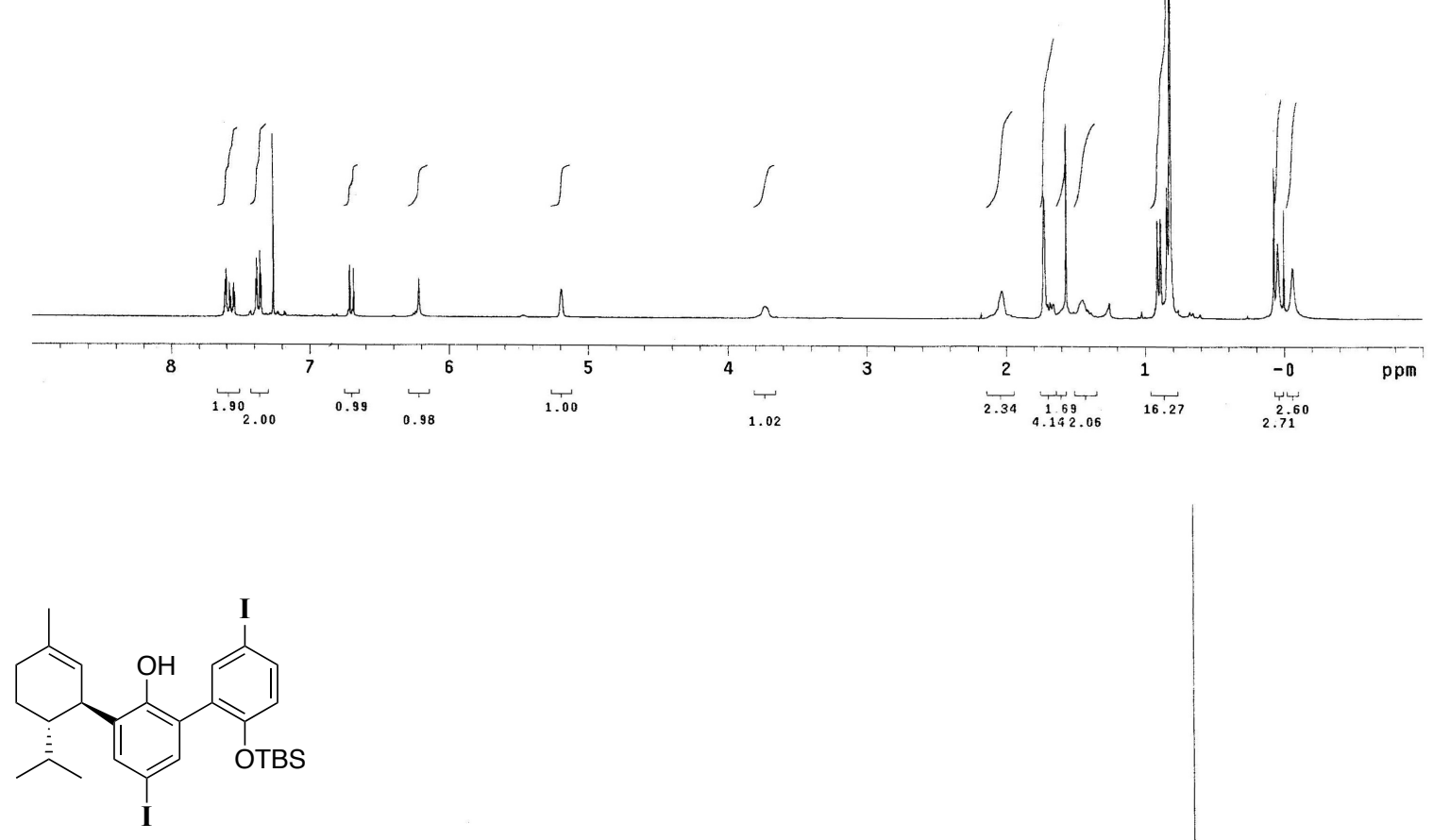

18

${ }^{13} \mathrm{C}$ NMR (75 MHz, $\mathrm{CDCl}_{3}$ )

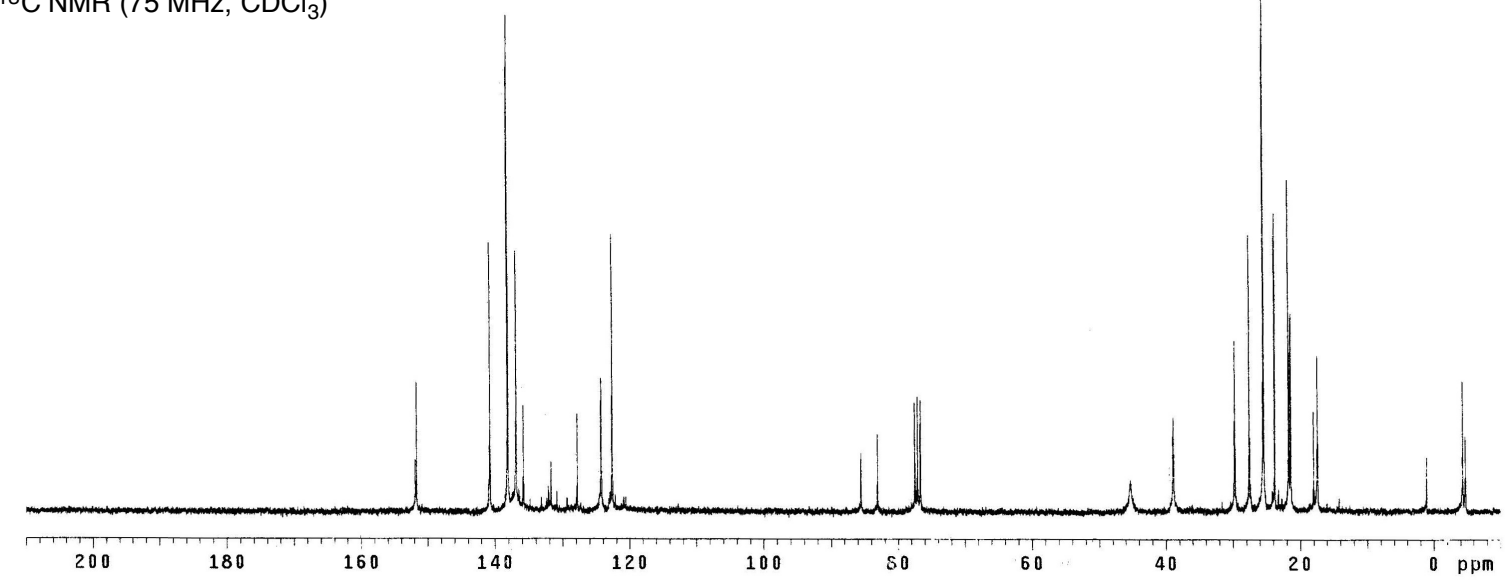




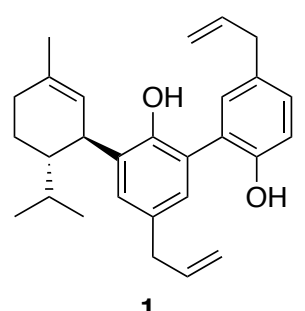

${ }^{1} \mathrm{H}$ NMR $\left(400 \mathrm{MHz}, \mathrm{CDCl}_{3}\right)$
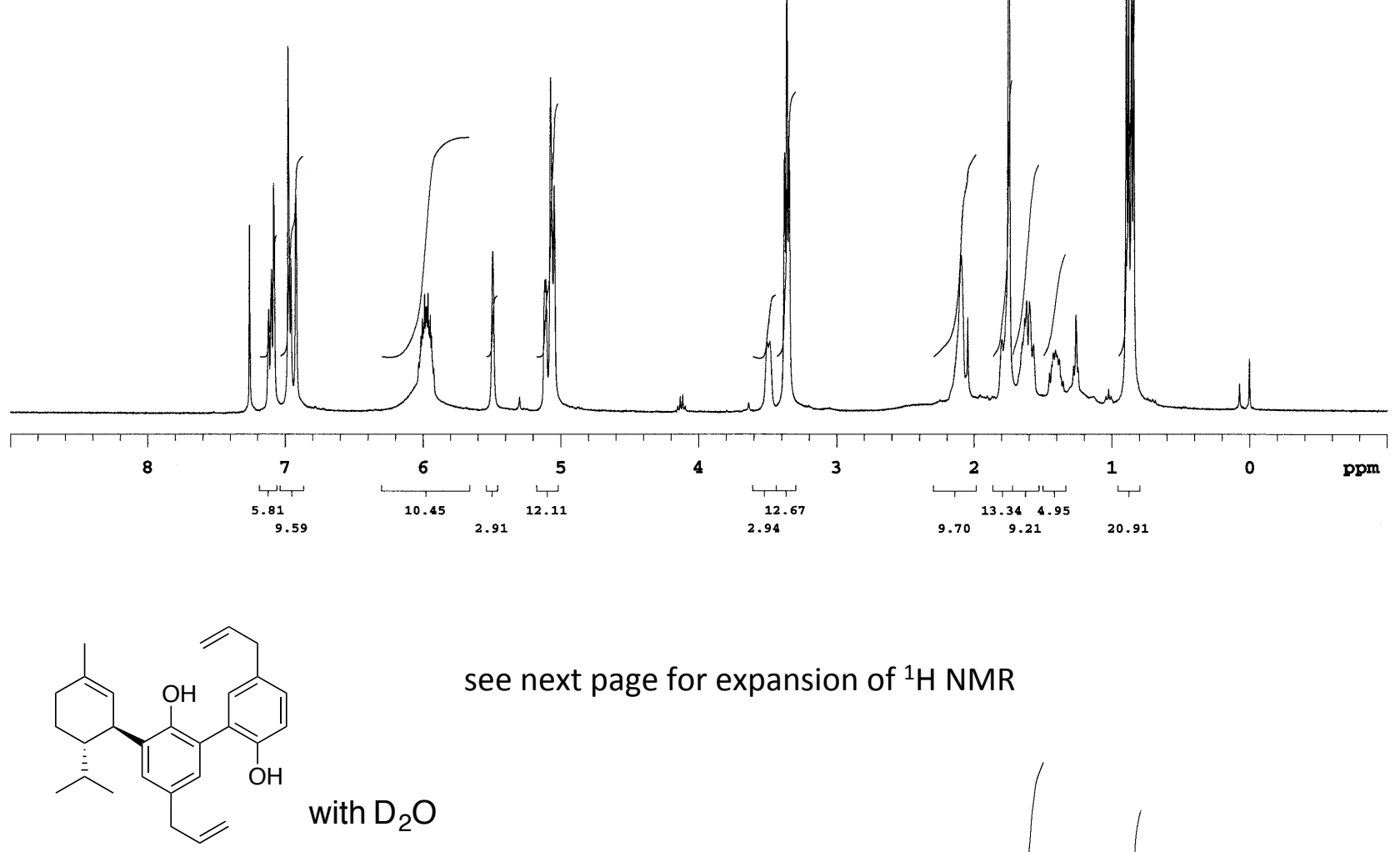

1

${ }^{1} \mathrm{H} \mathrm{NMR}\left(400 \mathrm{MHz}, \mathrm{CDCl}_{3}\right)$

see next page for expansion of ${ }^{1} \mathrm{H}$ NMR

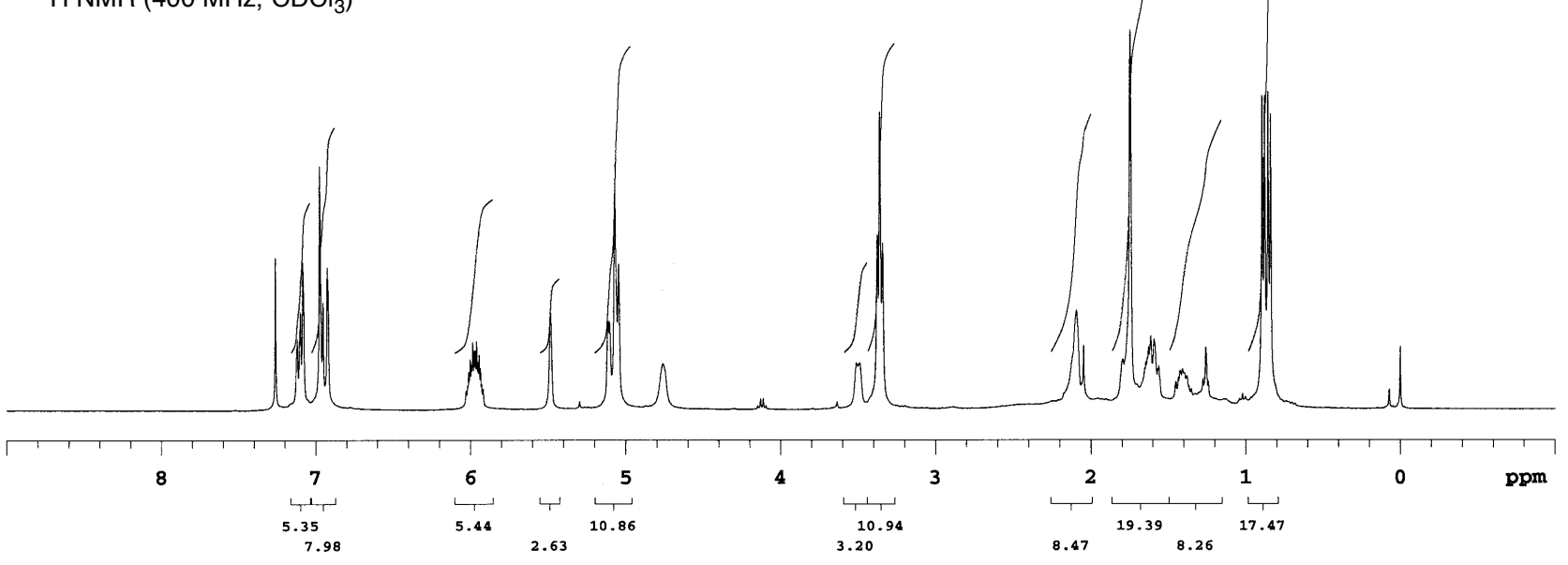




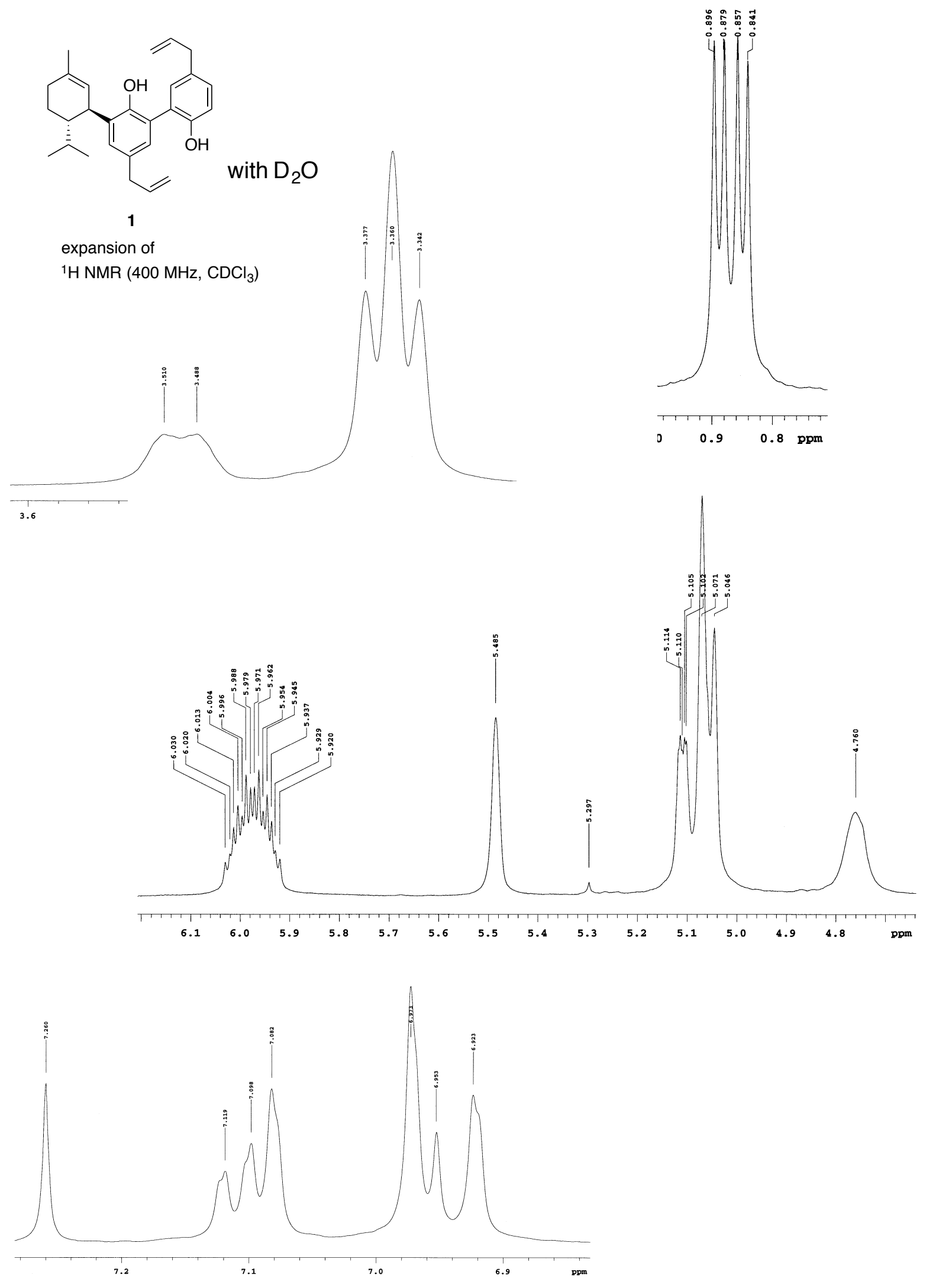




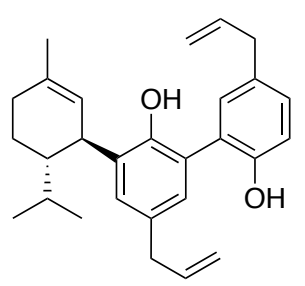

1

${ }^{13} \mathrm{C}$ NMR $\left(75 \mathrm{MHz}, \mathrm{CDCl}_{3}\right.$ )
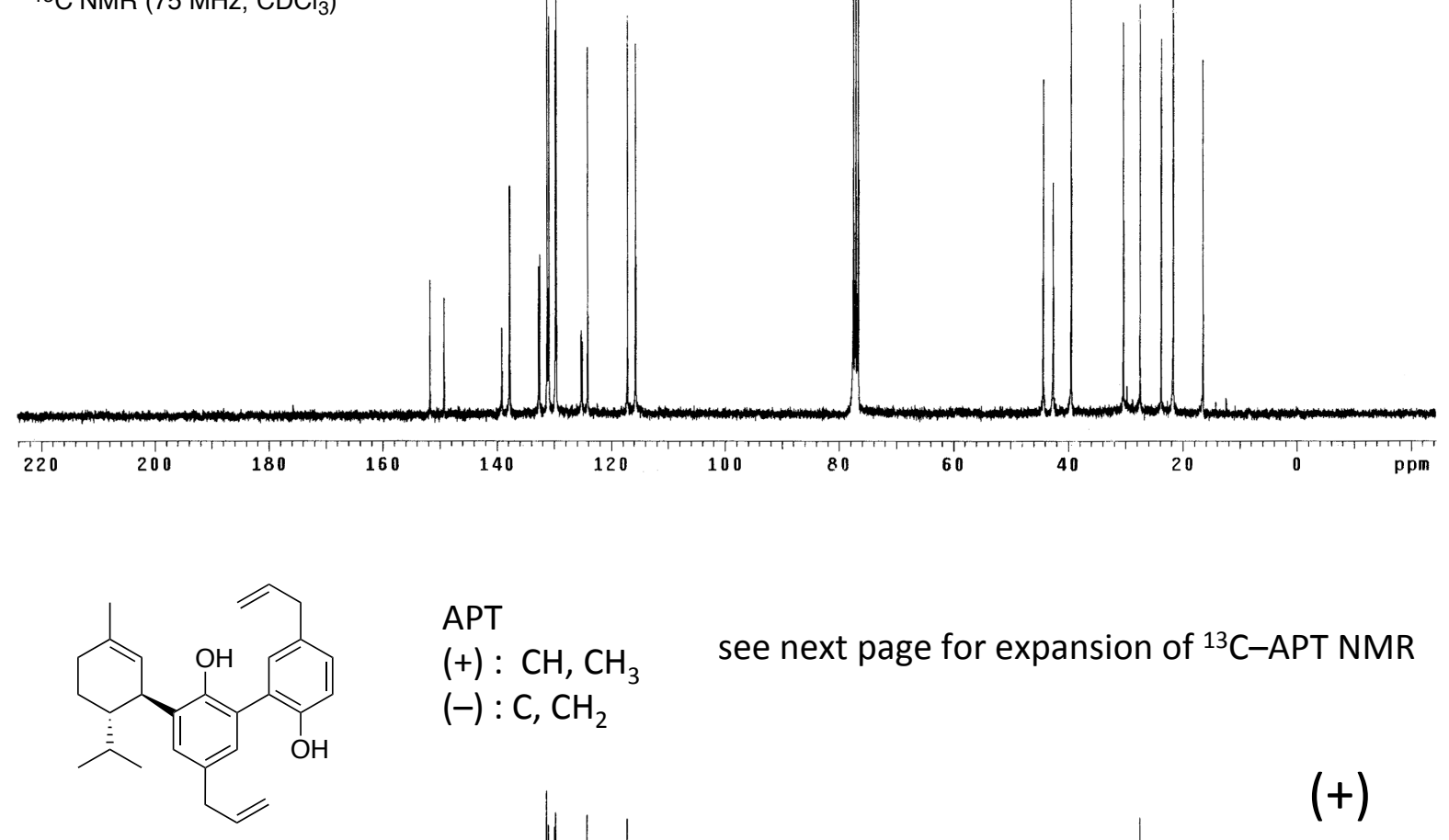

APT

(+) : $\mathrm{CH}, \mathrm{CH}_{3}$

see next page for expansion of ${ }^{13} \mathrm{C}-\mathrm{APT}$ NMR

$(-): \mathrm{C}, \mathrm{CH}_{2}$

$(+)$

${ }^{13} \mathrm{C}$-APT NMR $\left(75 \mathrm{MHz}, \mathrm{CDCl}_{3}\right)$

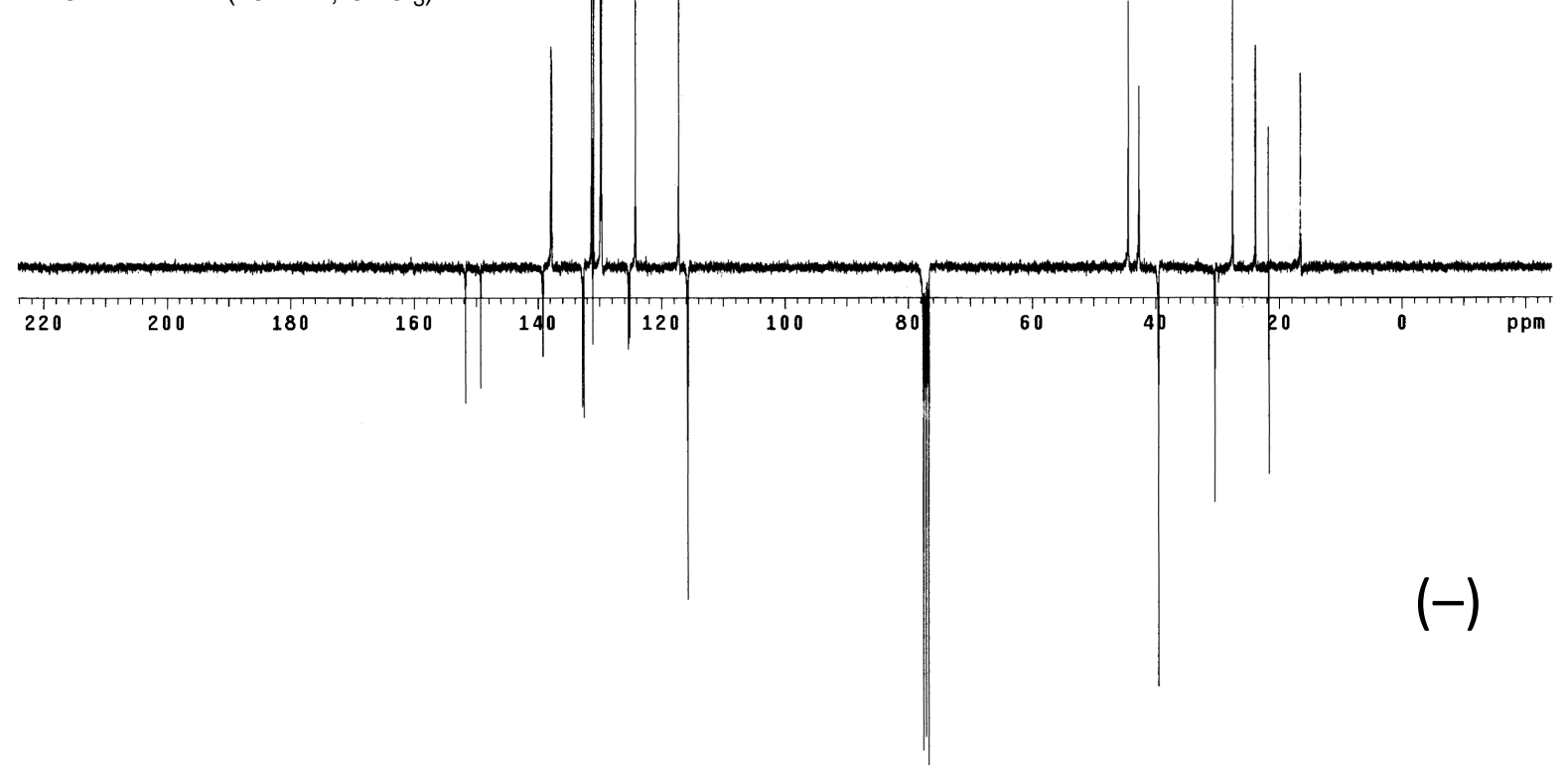


APT

(+) : $\mathrm{CH}, \mathrm{CH}_{3}$

$(-): \mathrm{C}, \mathrm{CH}_{2}$
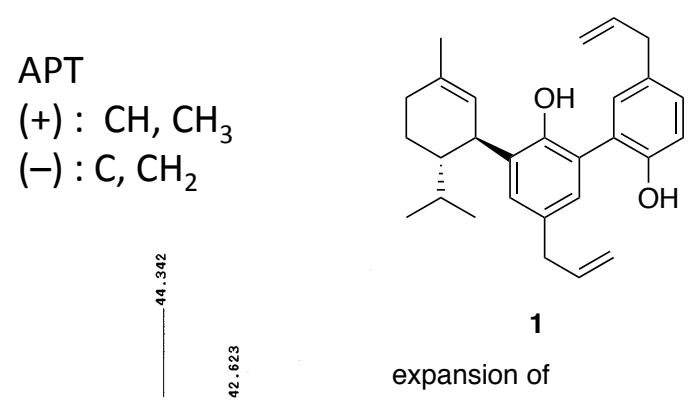

1

expansion of

${ }^{13} \mathrm{C}$-APT NMR (75 MHz, $\mathrm{CDCl}_{3}$ )

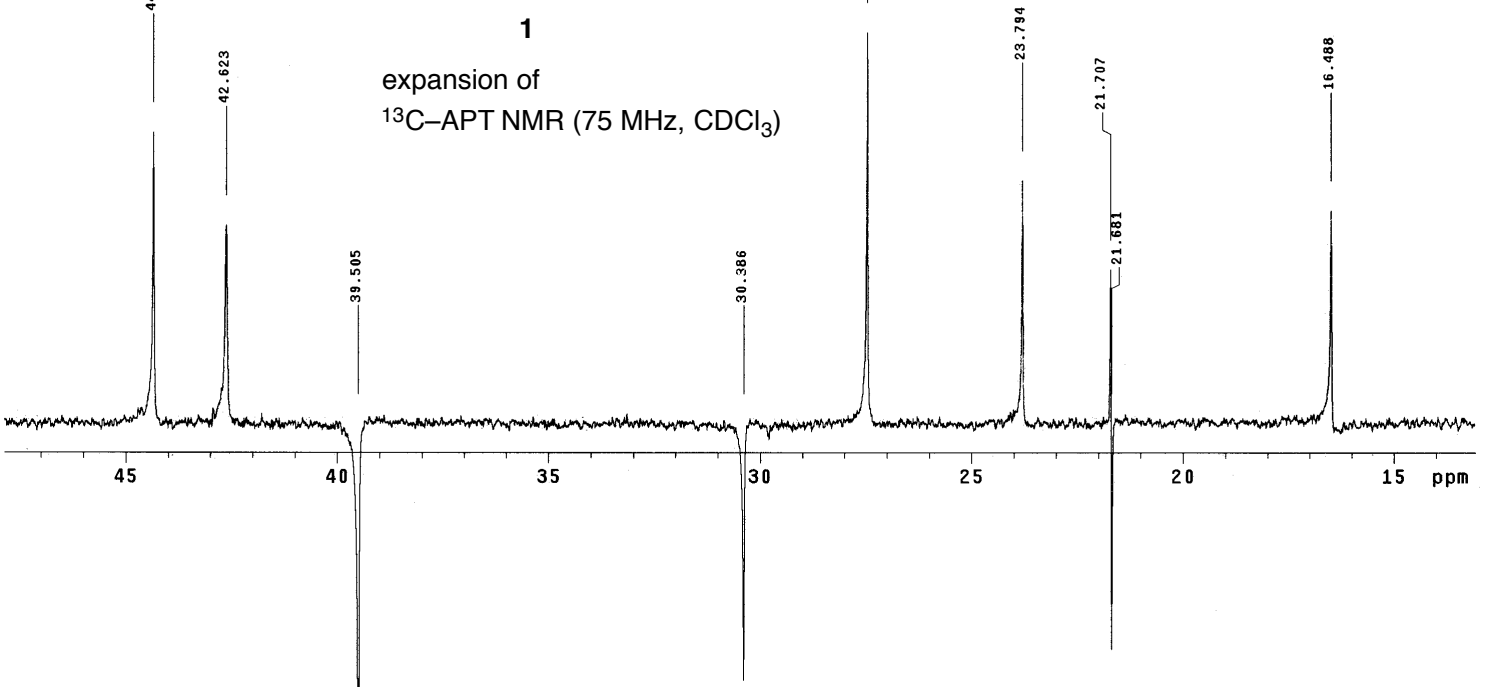

$(-)$

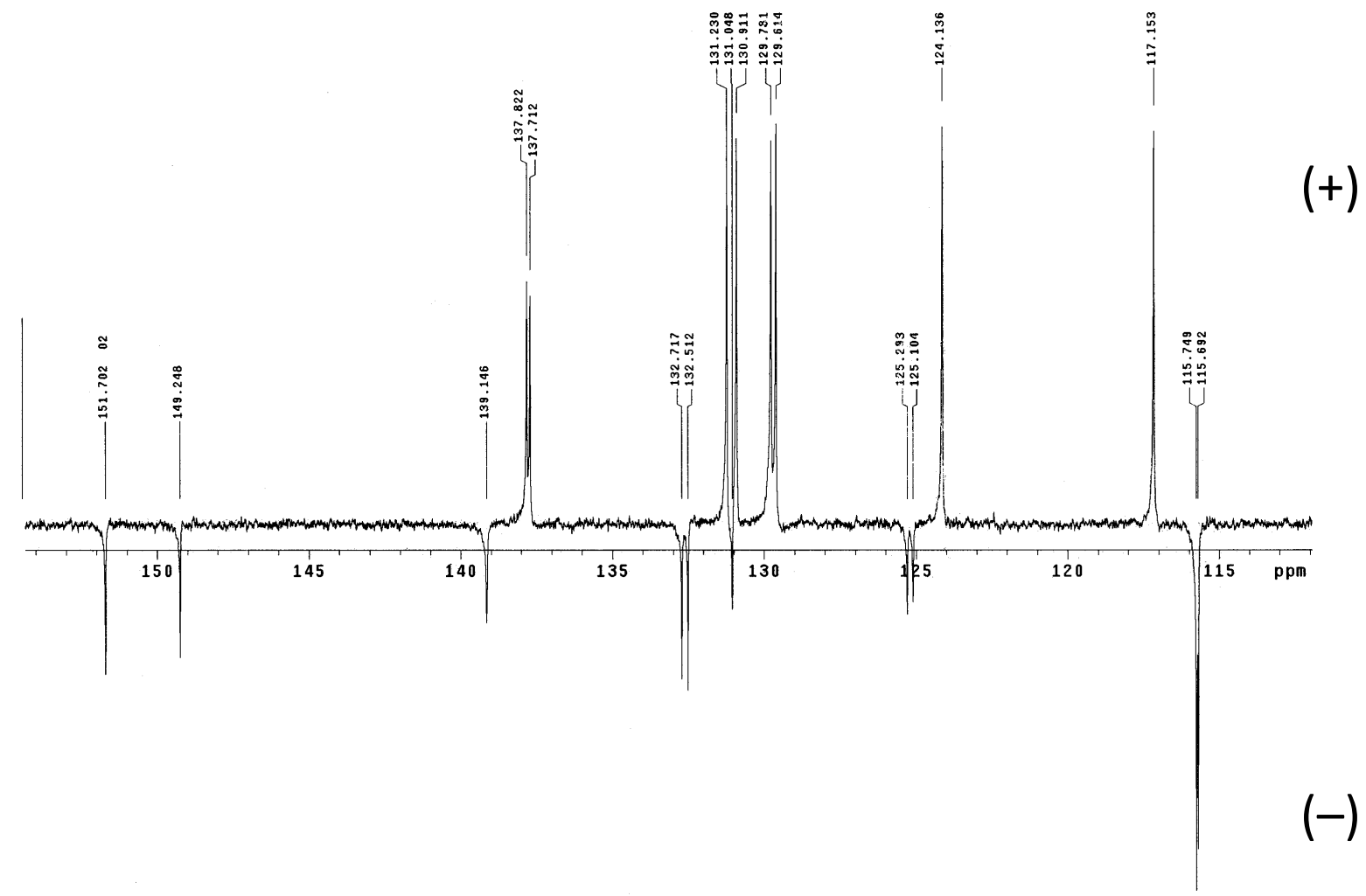




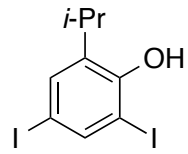

20

${ }^{1} \mathrm{H}$ NMR $\left(300 \mathrm{MHz}, \mathrm{CDCl}_{3}\right)$
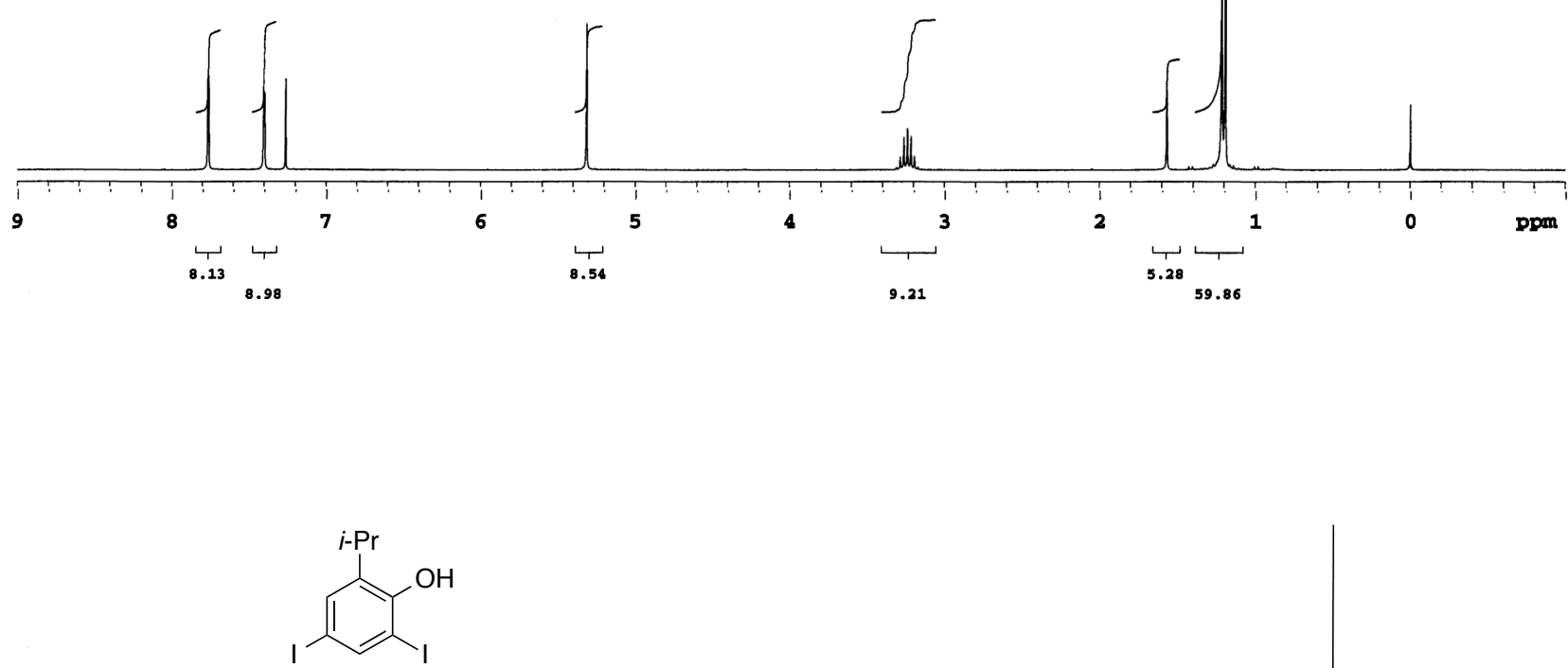

20

${ }^{13} \mathrm{C}$ NMR $\left(75 \mathrm{MHz}, \mathrm{CDCl}_{3}\right)$

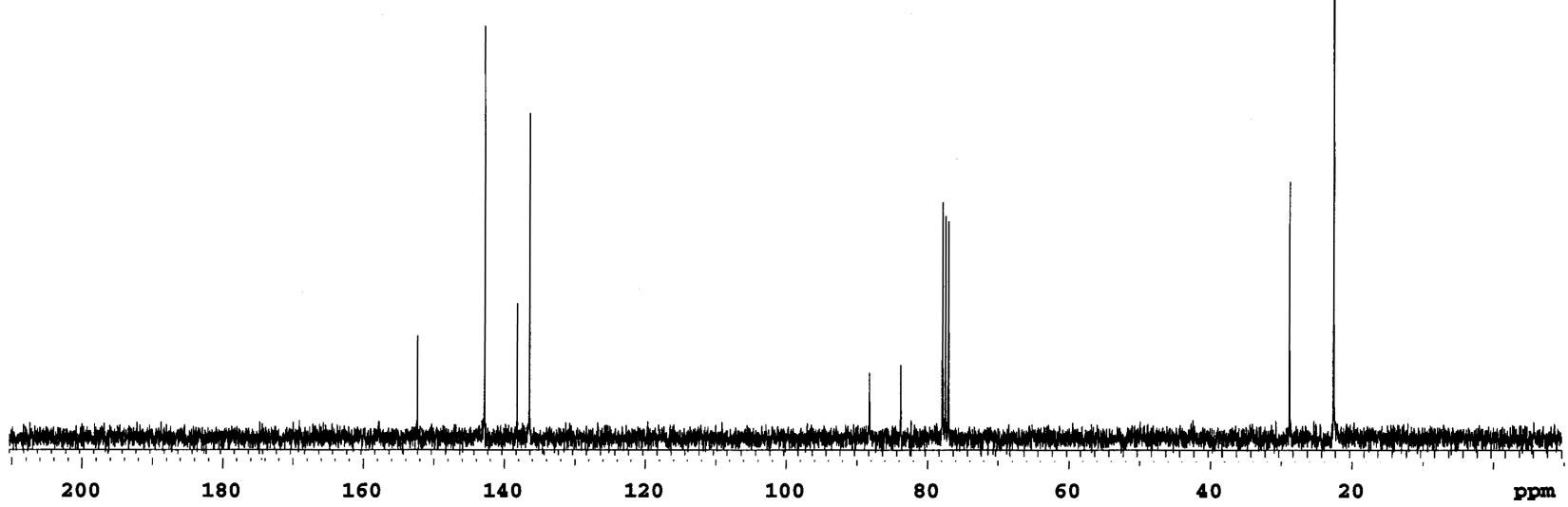


<smiles>O=[W]c1cc(I)cc(I)c1O</smiles>

24

${ }^{1} \mathrm{H}$ NMR $\left(300 \mathrm{MHz}, \mathrm{CDCl}_{3}\right)$
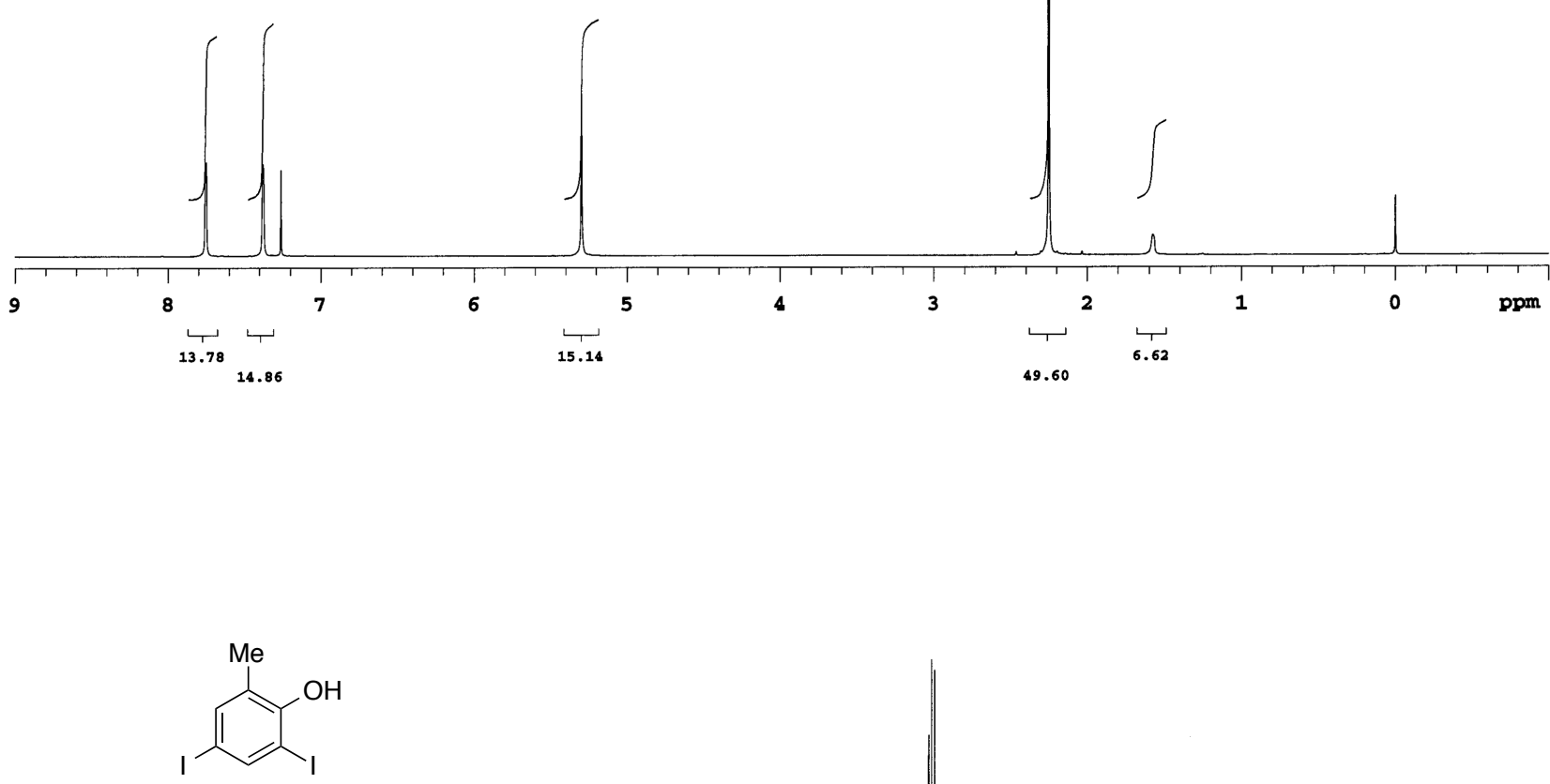

24

${ }^{13} \mathrm{C}$ NMR $\left(75 \mathrm{MHz}, \mathrm{CDCl}_{3}\right)$

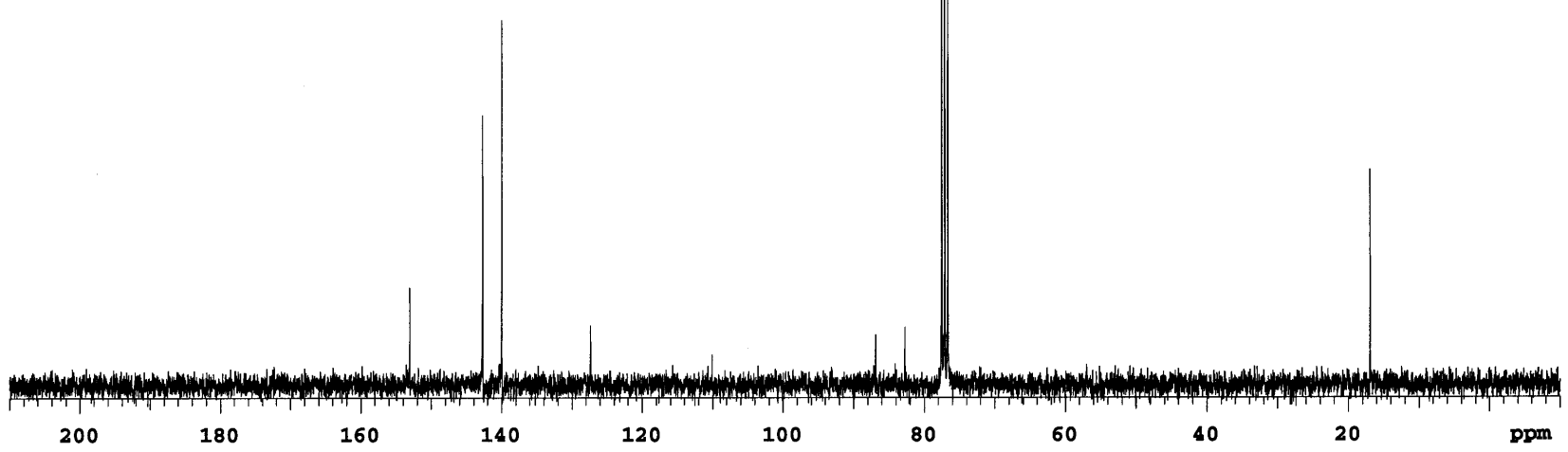


<smiles>CC(C)(C)c1cc(I)cc(I)c1O</smiles>

25

${ }^{1} \mathrm{H}$ NMR $\left(300 \mathrm{MHz}, \mathrm{CDCl}_{3}\right)$

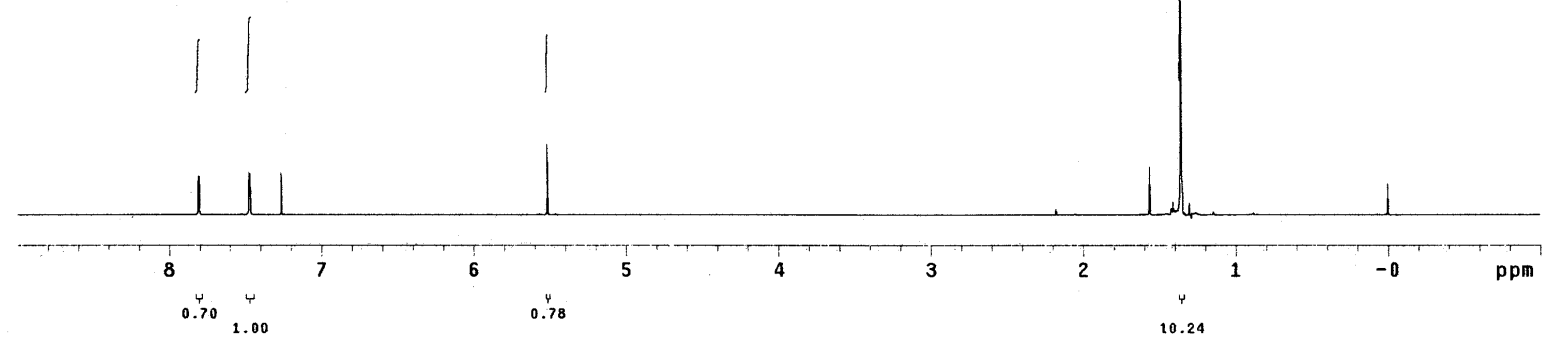<smiles>CC(C)(C)c1cc(I)cc(I)c1O</smiles>

25

${ }^{13} \mathrm{C} \mathrm{NMR}\left(75 \mathrm{MHz}, \mathrm{CDCl}_{3}\right)$

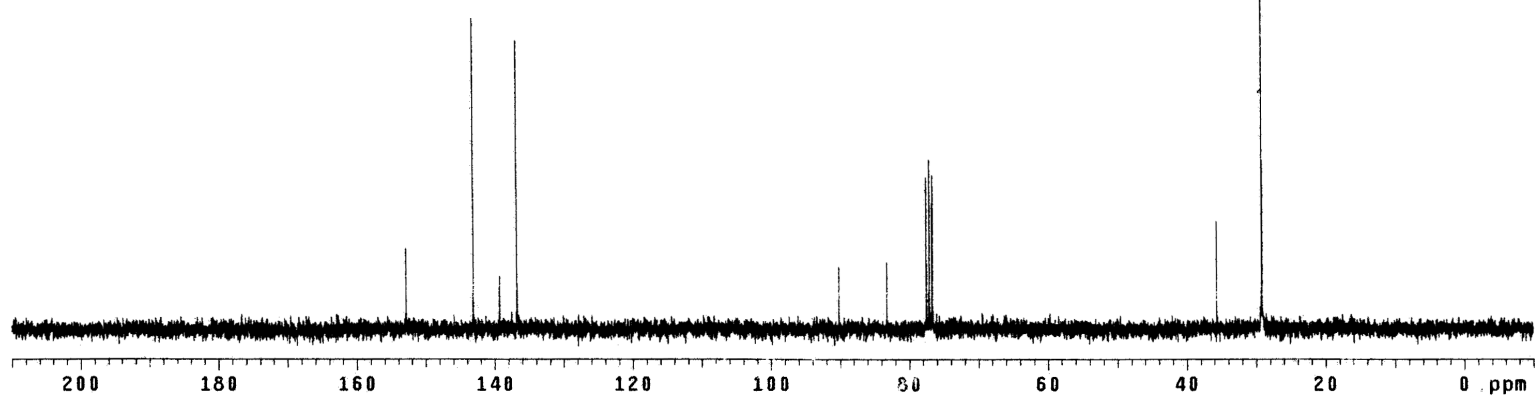



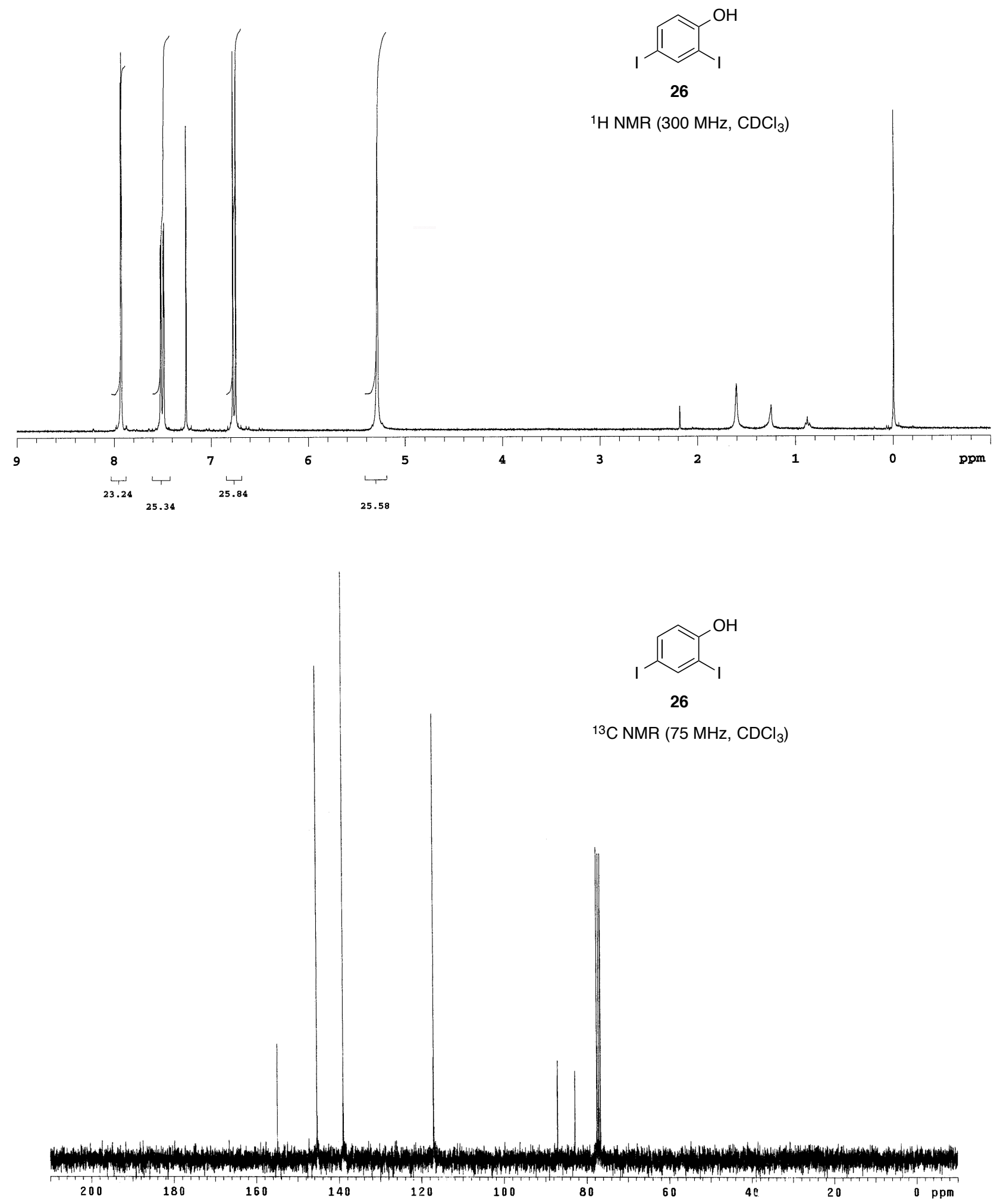


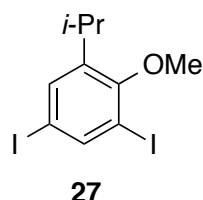

27

${ }^{1} \mathrm{H}$ NMR $\left(300 \mathrm{MHz}, \mathrm{CDCl}_{3}\right)$
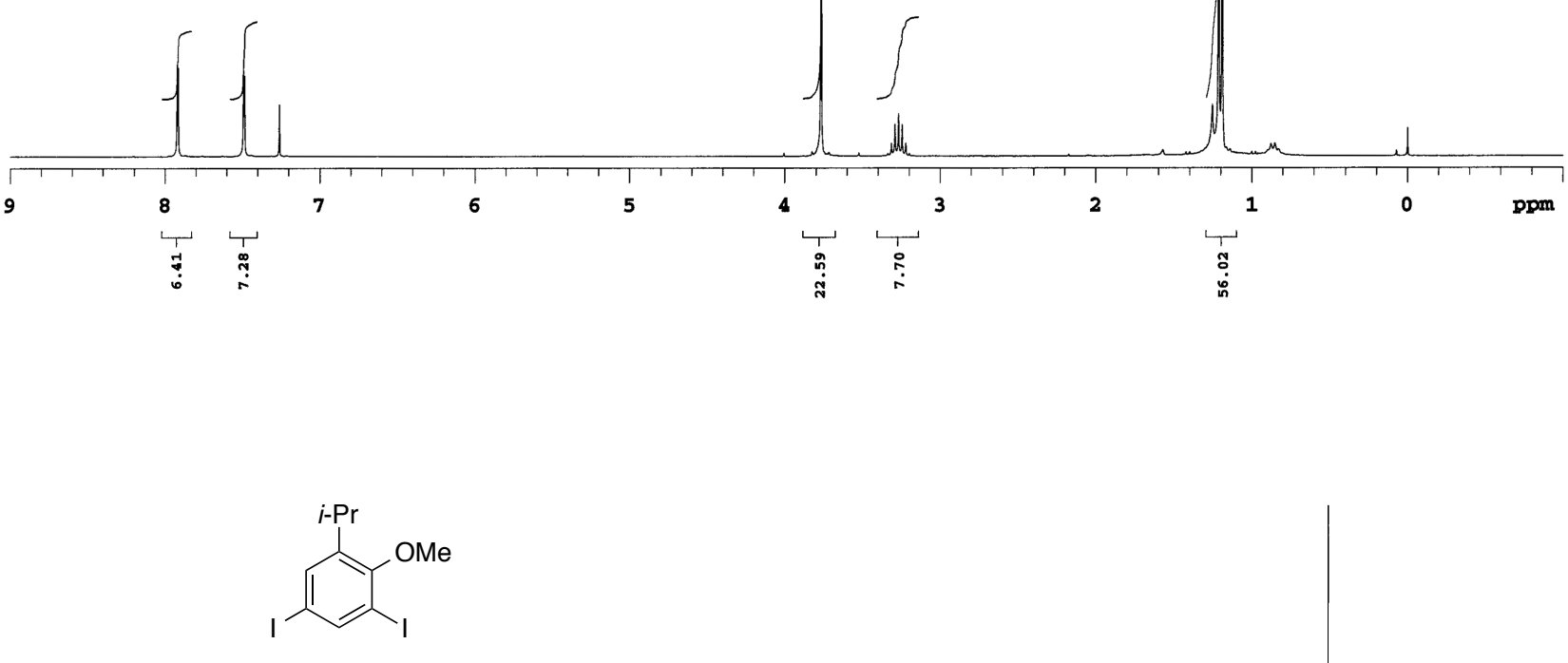

27

${ }^{13} \mathrm{C}$ NMR $\left(75 \mathrm{MHz}, \mathrm{CDCl}_{3}\right)$

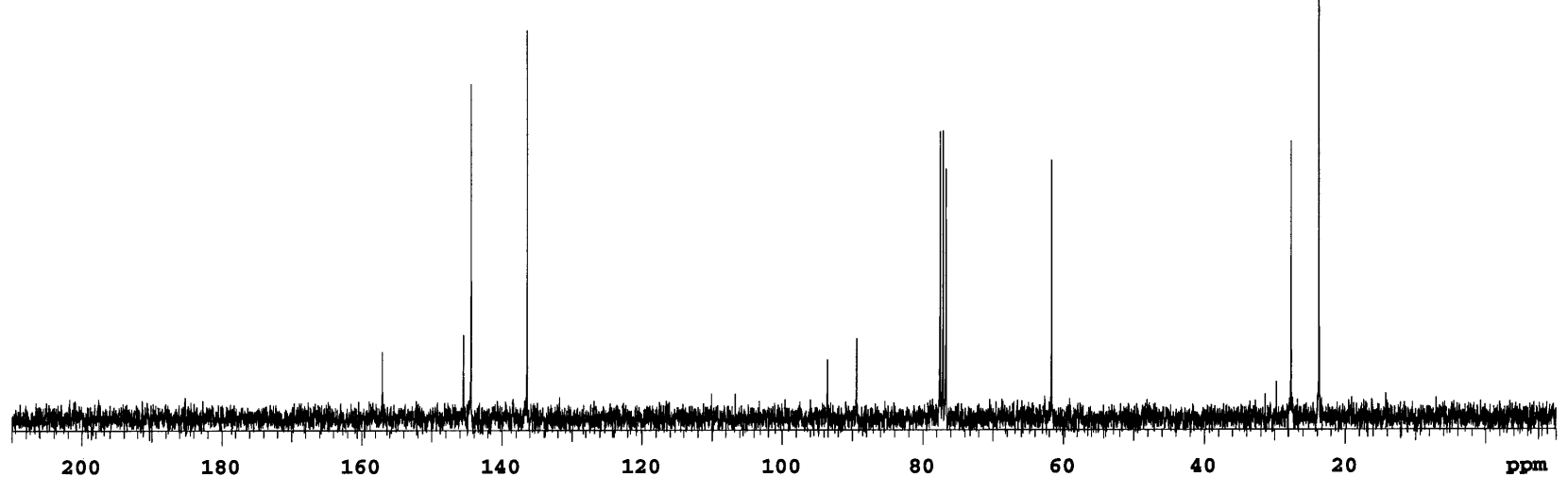



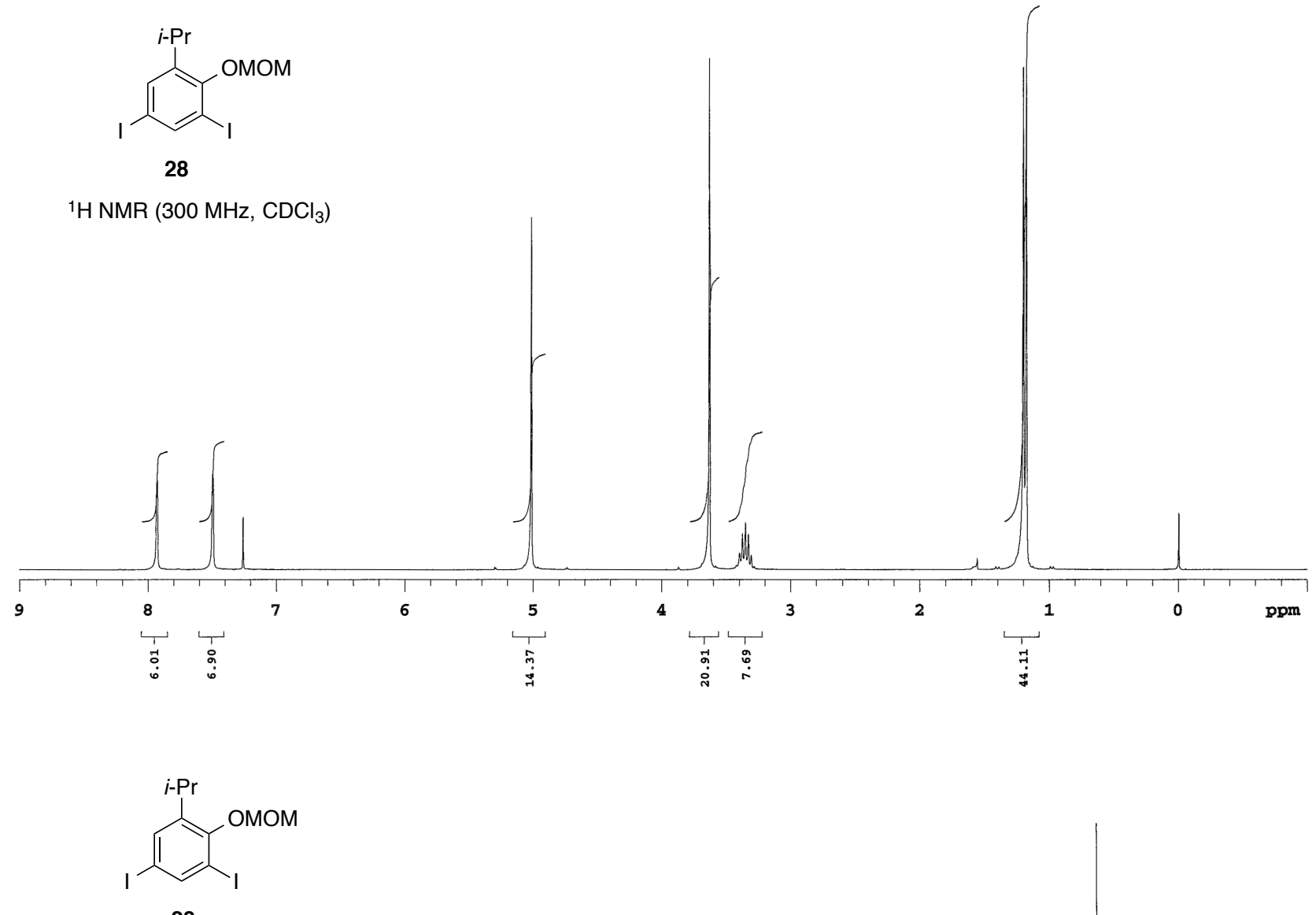

${ }^{13} \mathrm{C}$ NMR $\left(75 \mathrm{MHz}, \mathrm{CDCl}_{3}\right)$

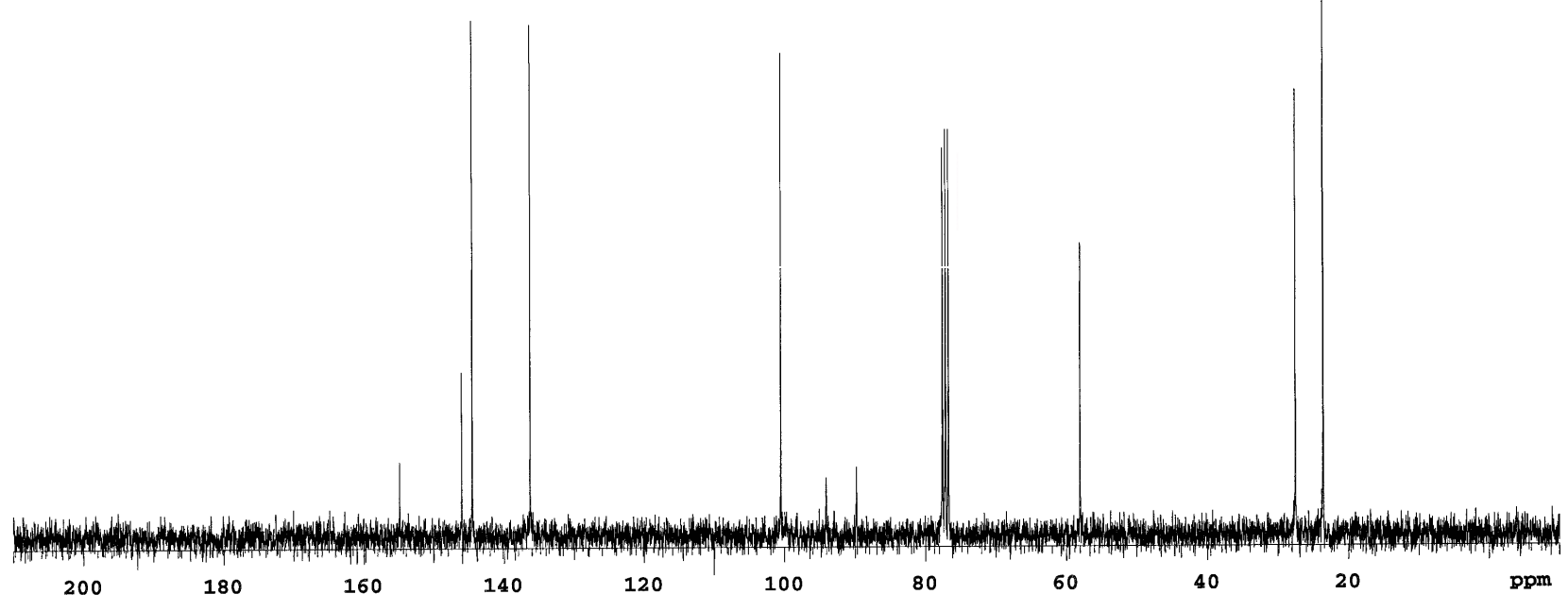

S37 


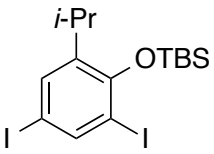

29

${ }^{1} \mathrm{H}$ NMR (300 MHz, $\left.\mathrm{CDCl}_{3}\right)$
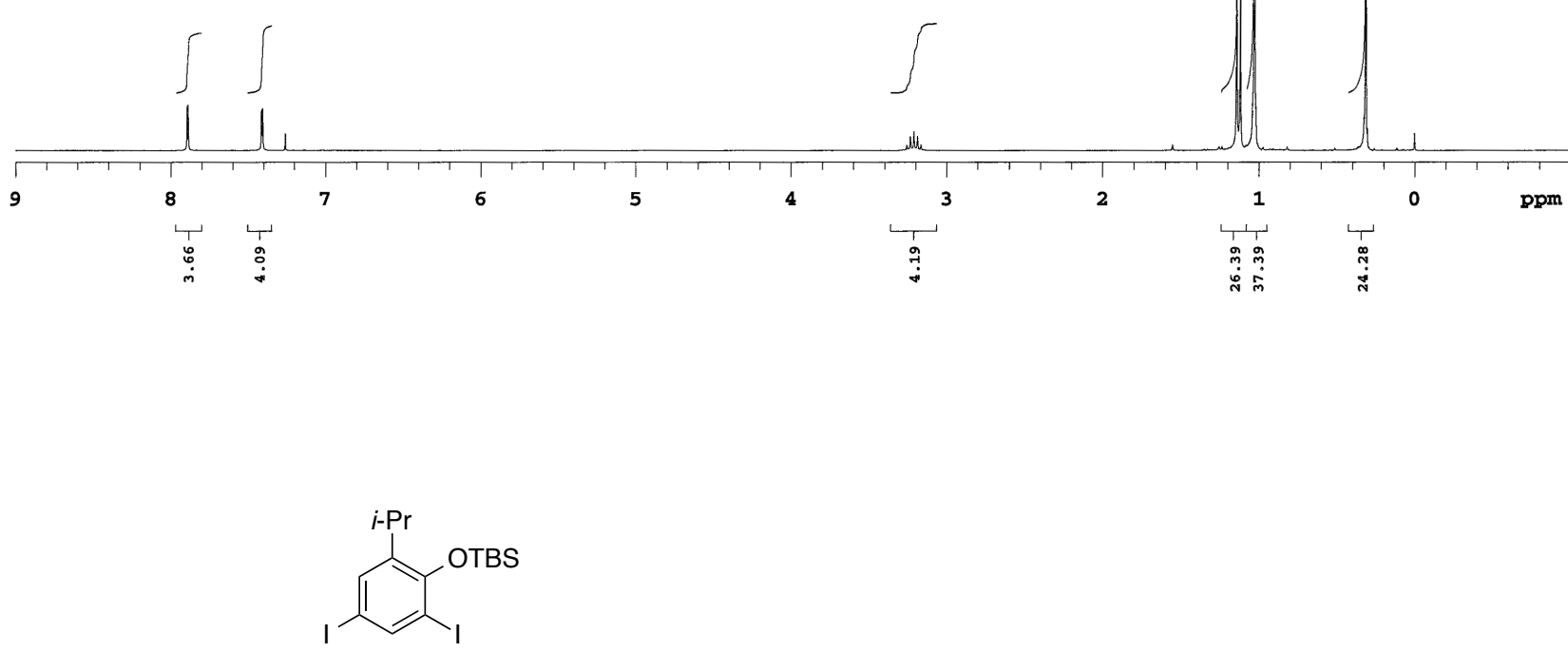

29

${ }^{13} \mathrm{C}$ NMR $\left(75 \mathrm{MHz}, \mathrm{CDCl}_{3}\right)$ 
NMR calculation of a product ratio

For example, Table 1, entry 1 and Table S1, entry S1

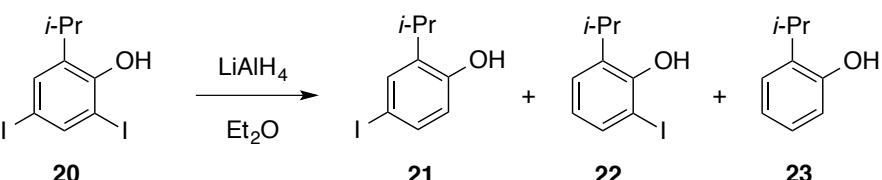

20

21

22

23

${ }^{1} \mathrm{H} \mathrm{NMR}\left(300 \mathrm{MHz}, \mathrm{CDCl}_{3}\right)$ of crude product
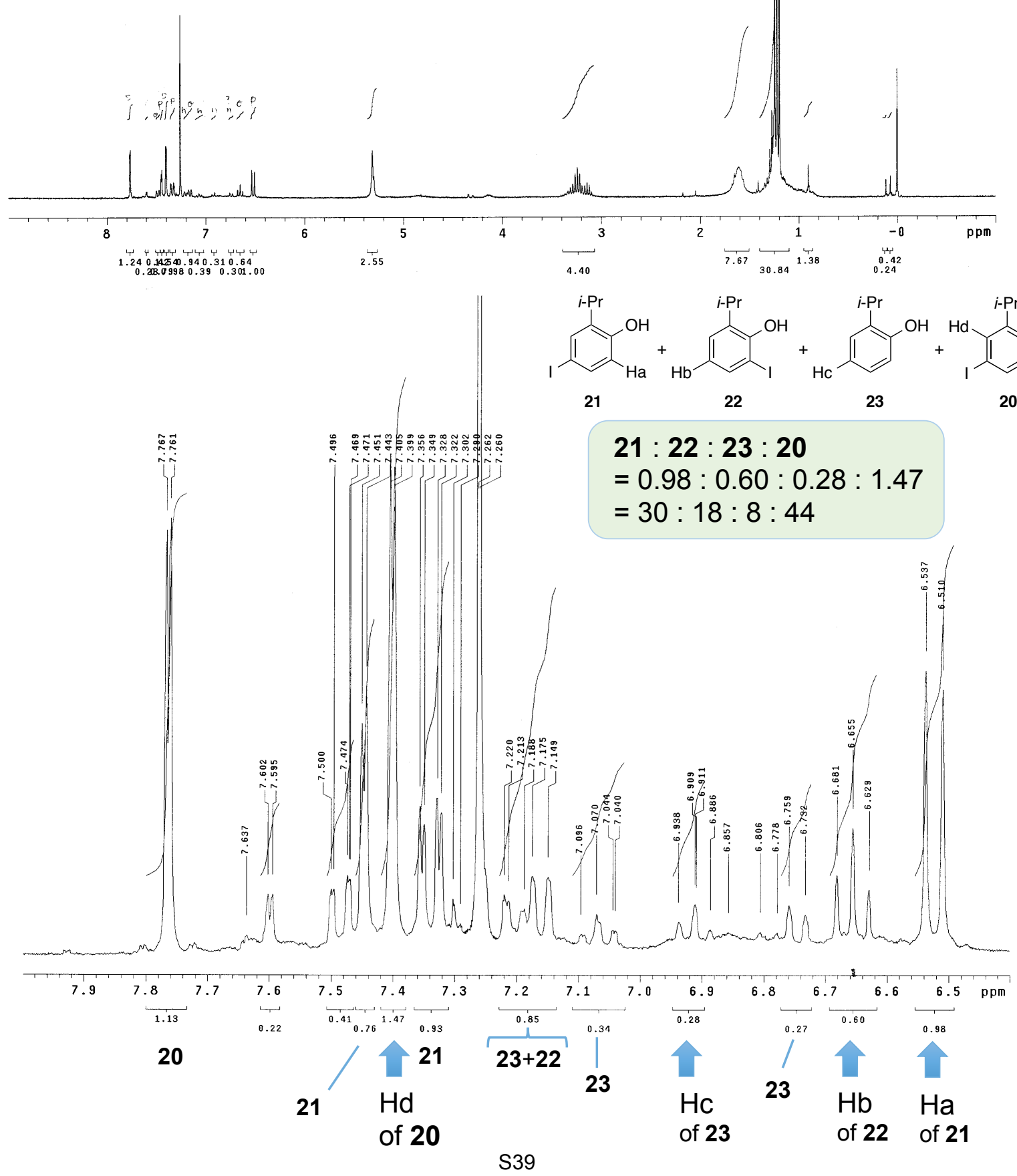


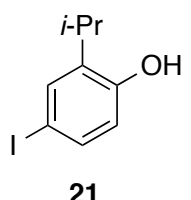

${ }^{1} \mathrm{H}$ NMR $\left(300 \mathrm{MHz}, \mathrm{CDCl}_{3}\right)$
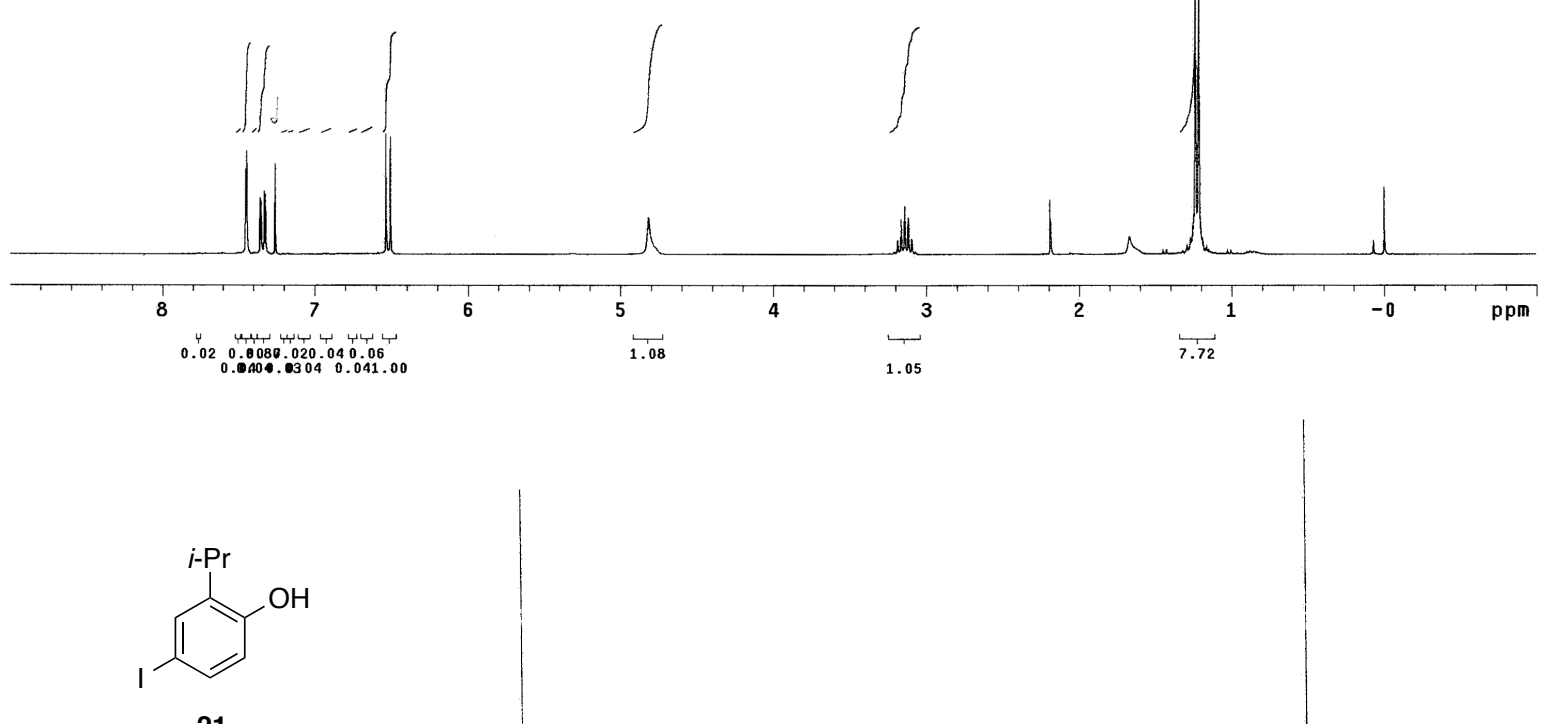

${ }^{13} \mathrm{C}$ NMR $\left(75 \mathrm{MHz}, \mathrm{CDCl}_{3}\right)$

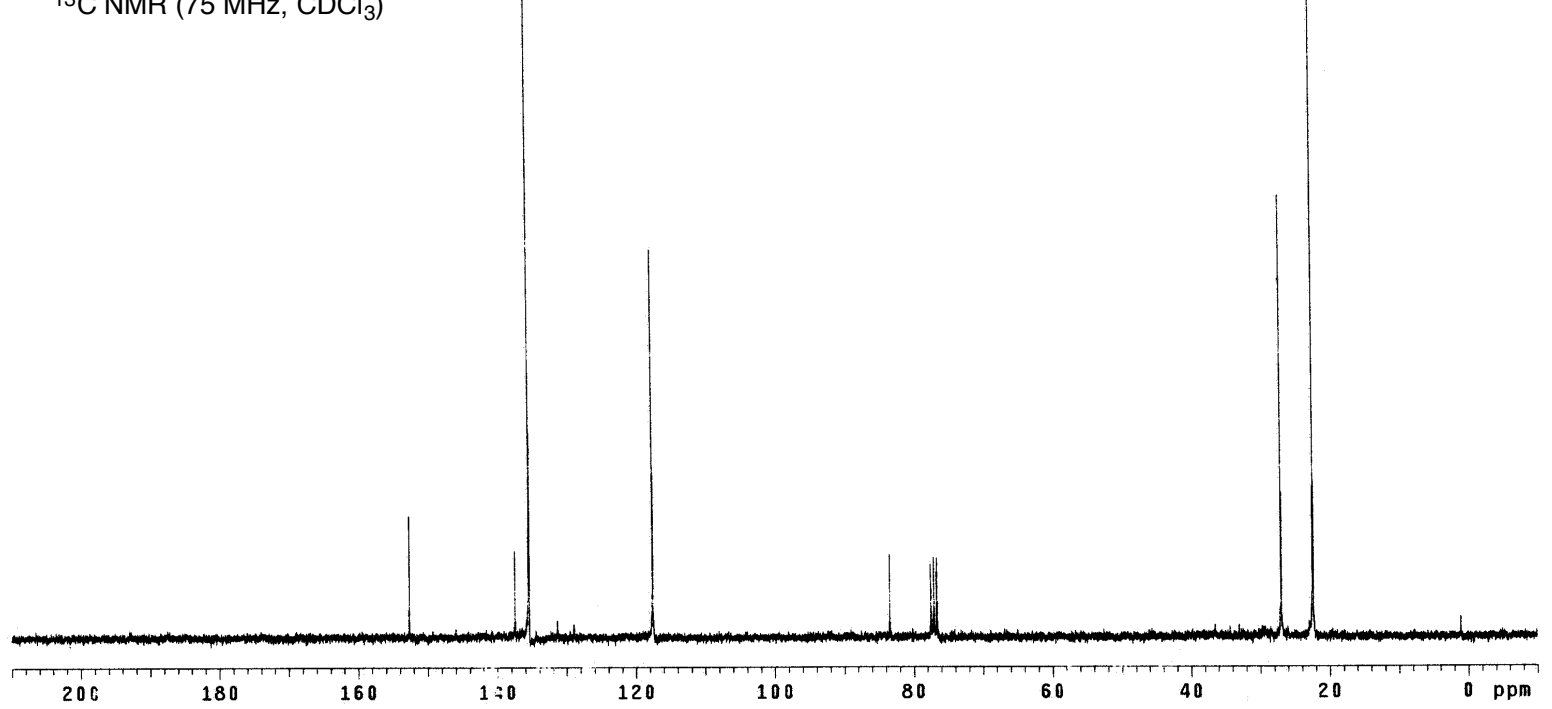




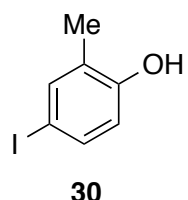

30

${ }^{1} \mathrm{H}$ NMR $\left(300 \mathrm{MHz}, \mathrm{CDCl}_{3}\right)$
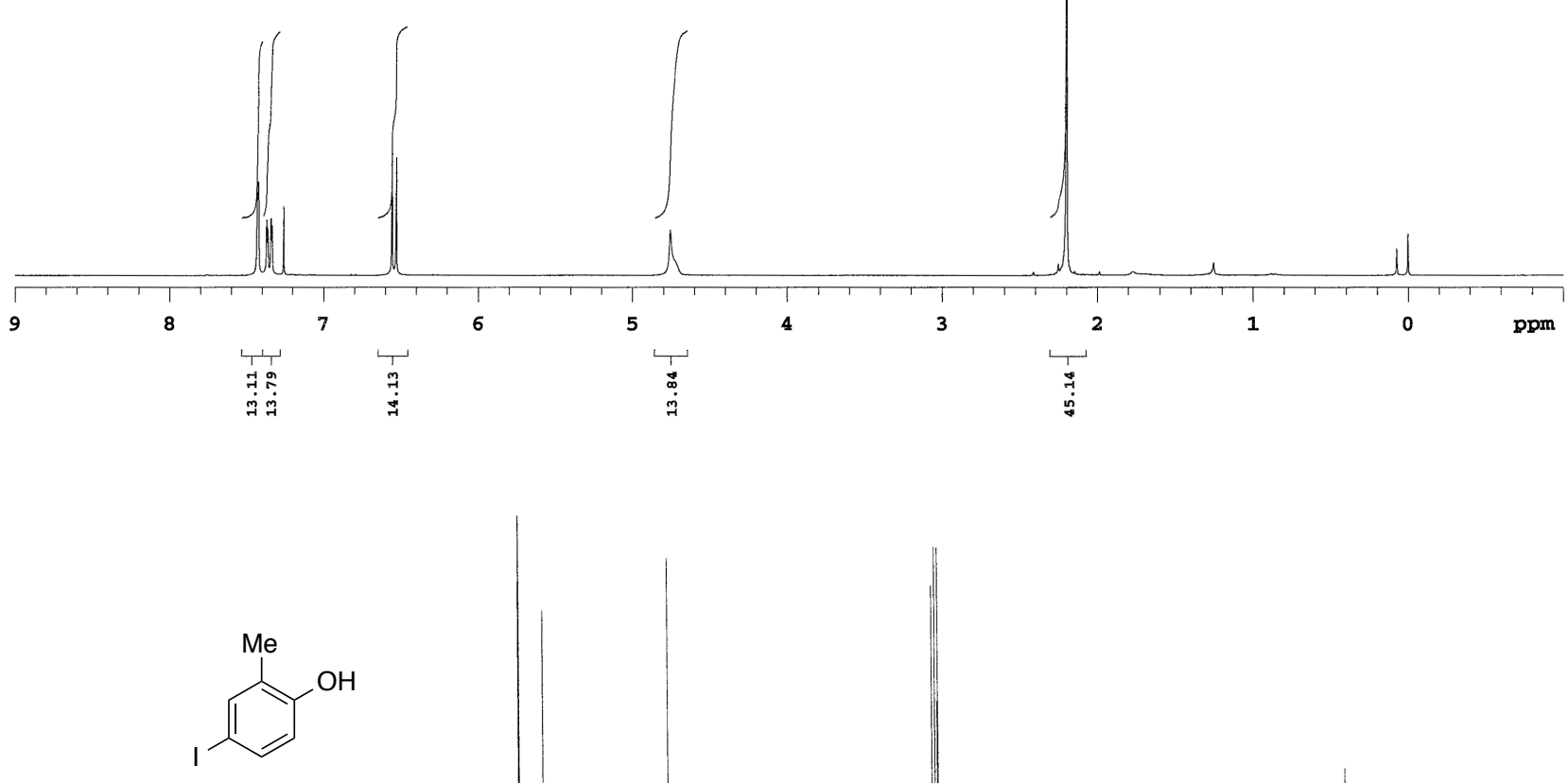

30

${ }^{13} \mathrm{C}$ NMR $\left(75 \mathrm{MHz}, \mathrm{CDCl}_{3}\right.$ )

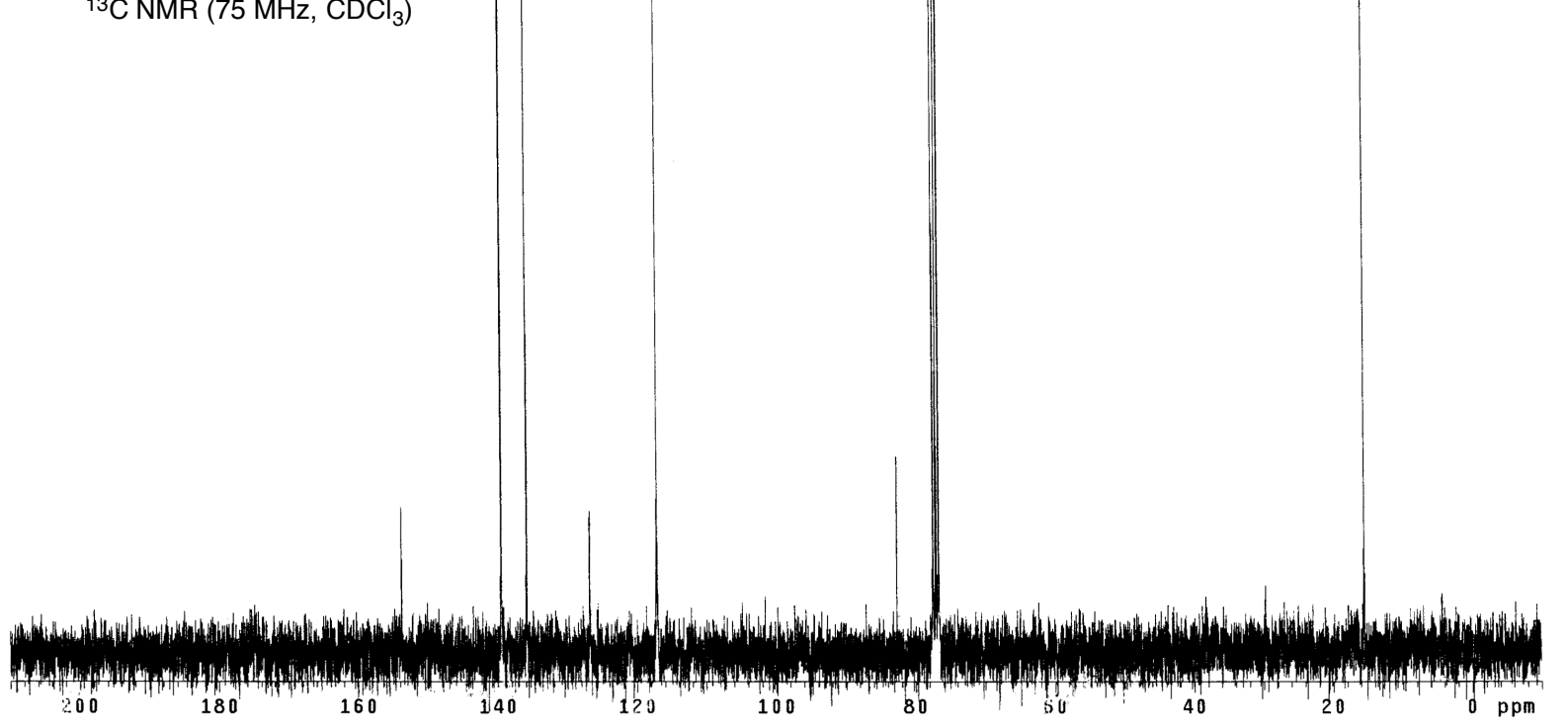




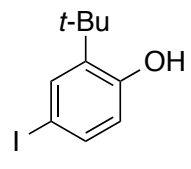

31

${ }^{1} \mathrm{H}$ NMR $\left(300 \mathrm{MHz}, \mathrm{CDCl}_{3}\right)$
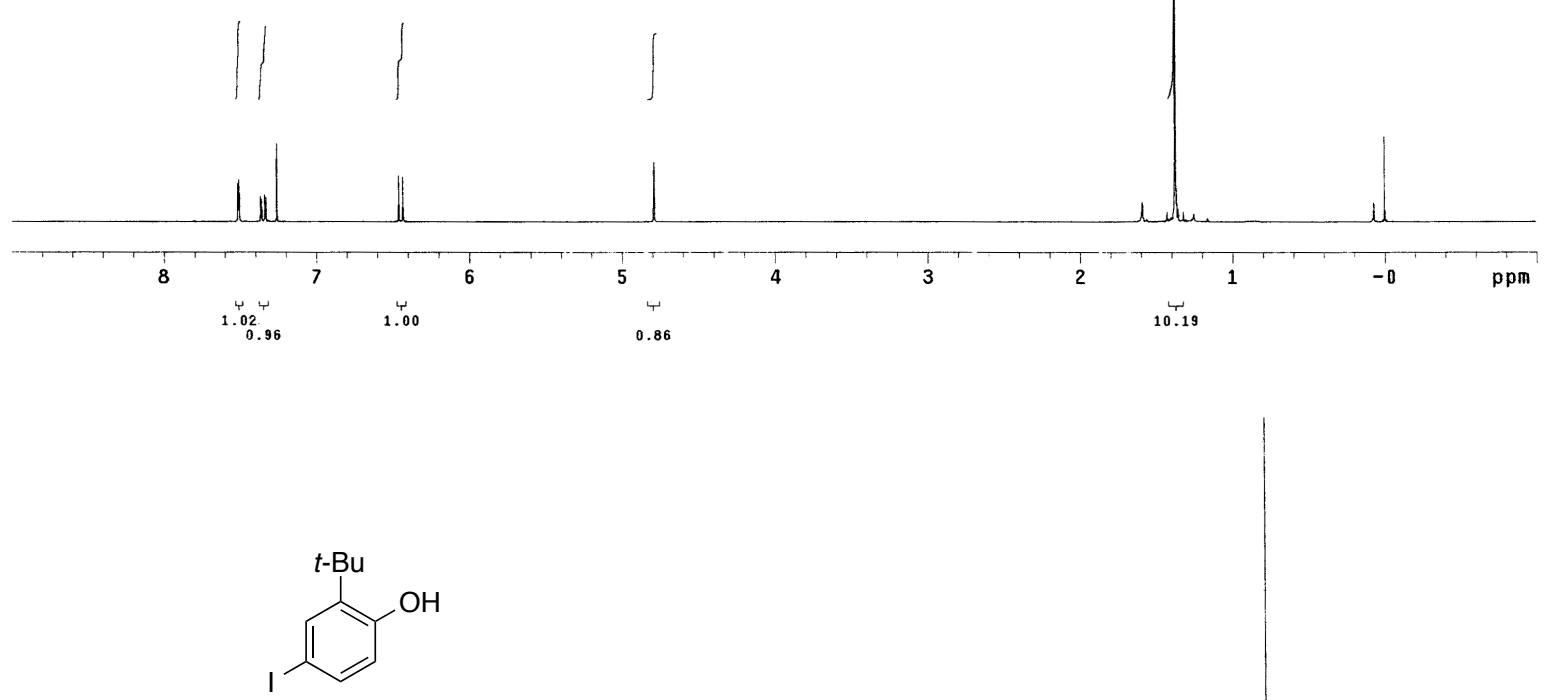

31

${ }^{13} \mathrm{C} \mathrm{NMR}\left(75 \mathrm{MHz}, \mathrm{CDCl}_{3}\right)$

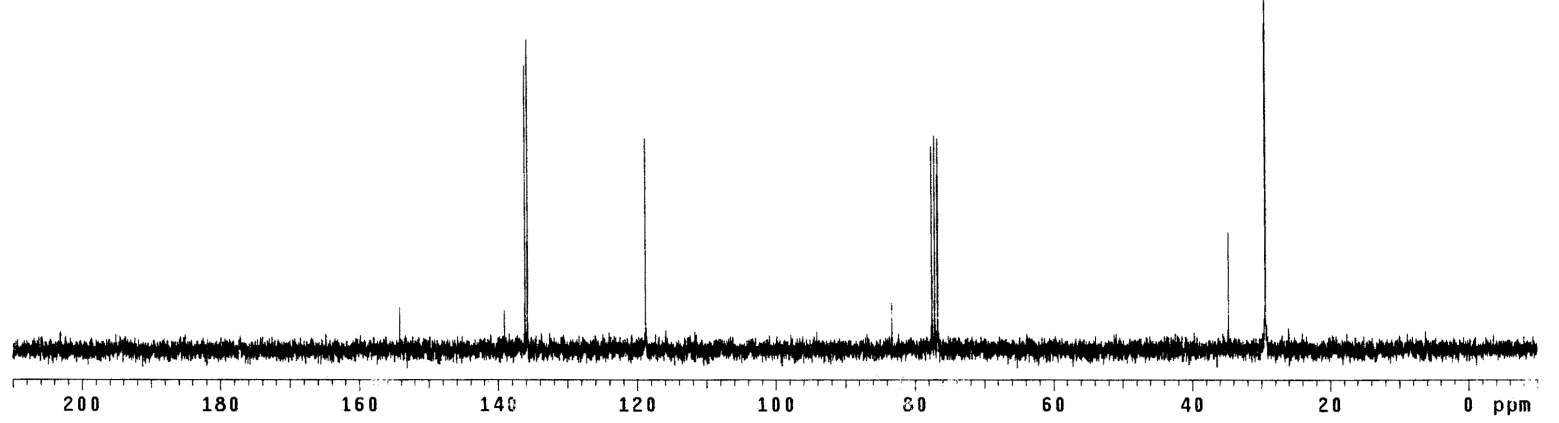




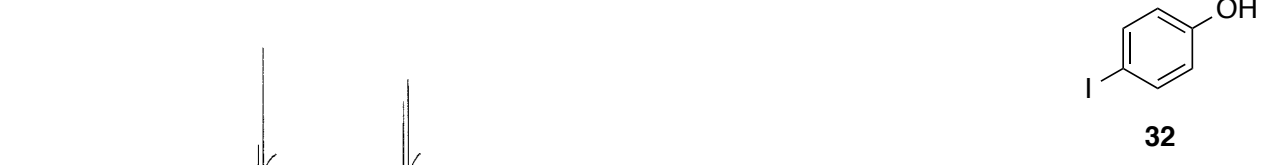

${ }^{1} \mathrm{H}$ NMR $\left(300 \mathrm{MHz}, \mathrm{CDCl}_{3}\right)$

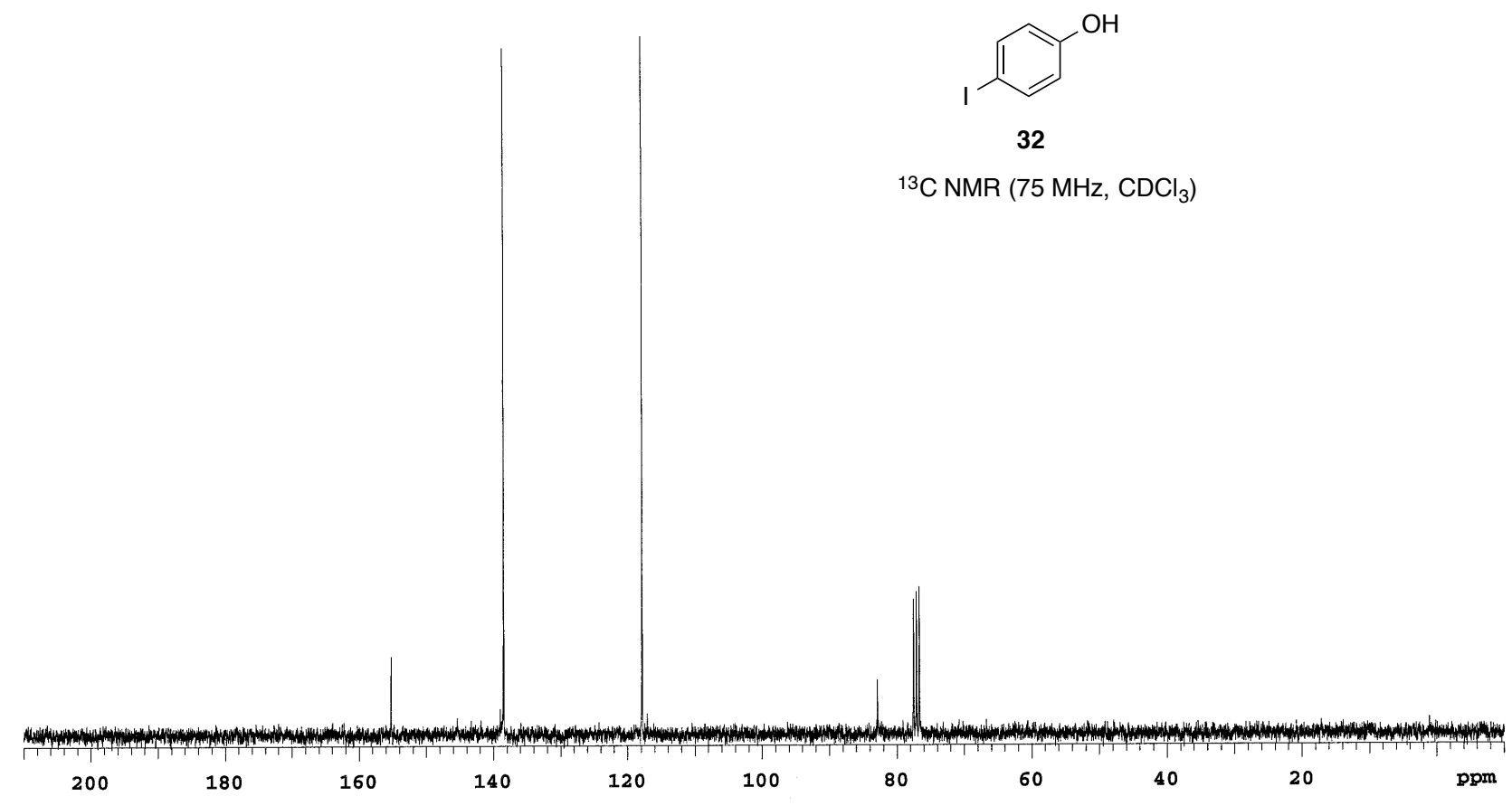




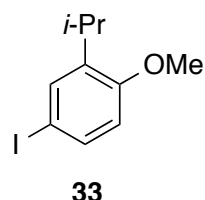

33

${ }^{1} \mathrm{H}$ NMR $\left(300 \mathrm{MHz}, \mathrm{CDCl}_{3}\right)$
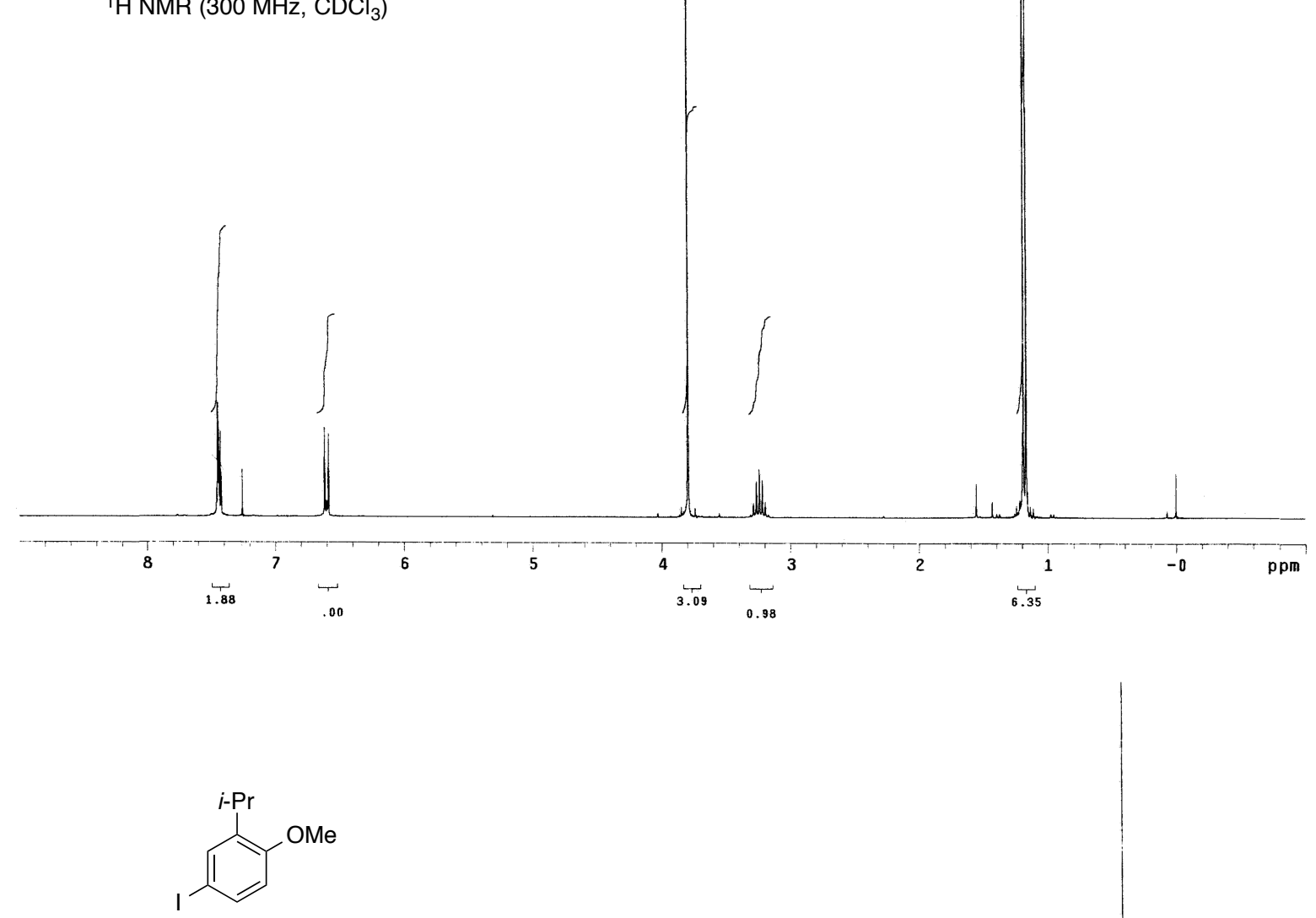

33

${ }^{13} \mathrm{C}$ NMR $\left(75 \mathrm{MHz}, \mathrm{CDCl}_{3}\right)$

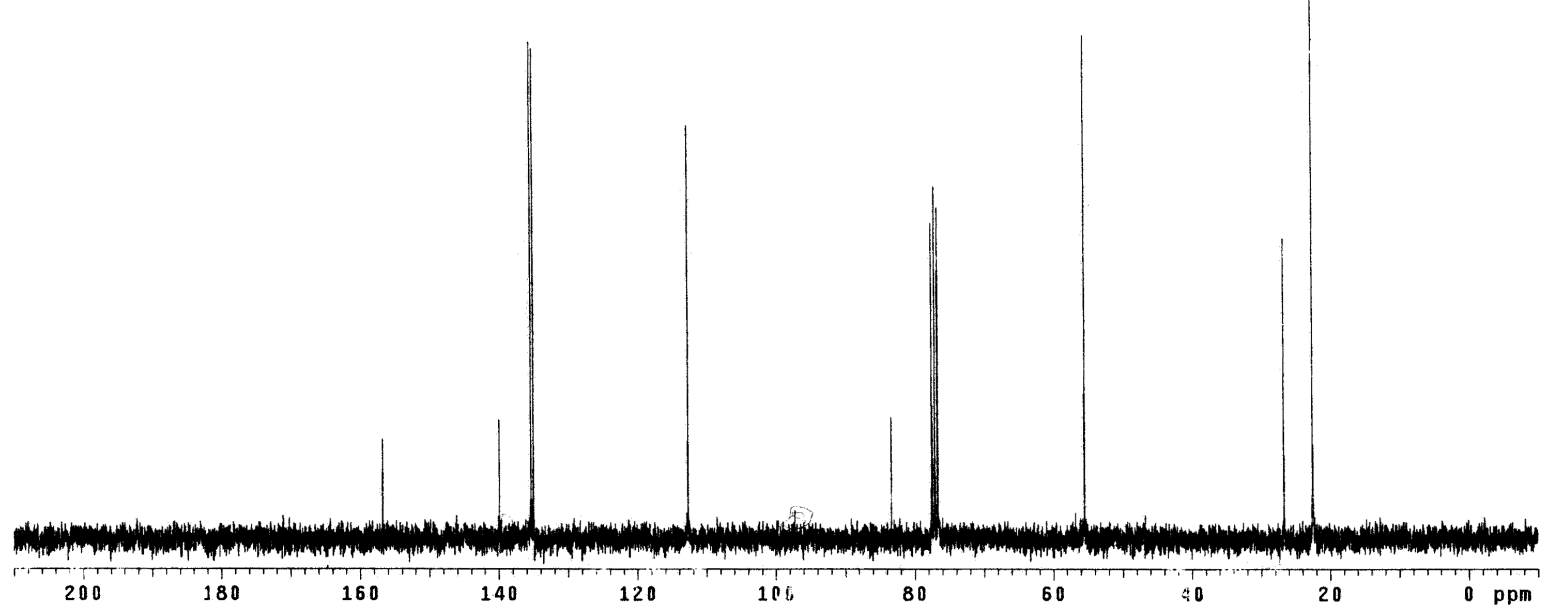




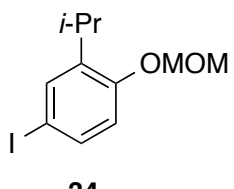

34

${ }^{1} \mathrm{H}$ NMR $\left(300 \mathrm{MHz}, \mathrm{CDCl}_{3}\right)$
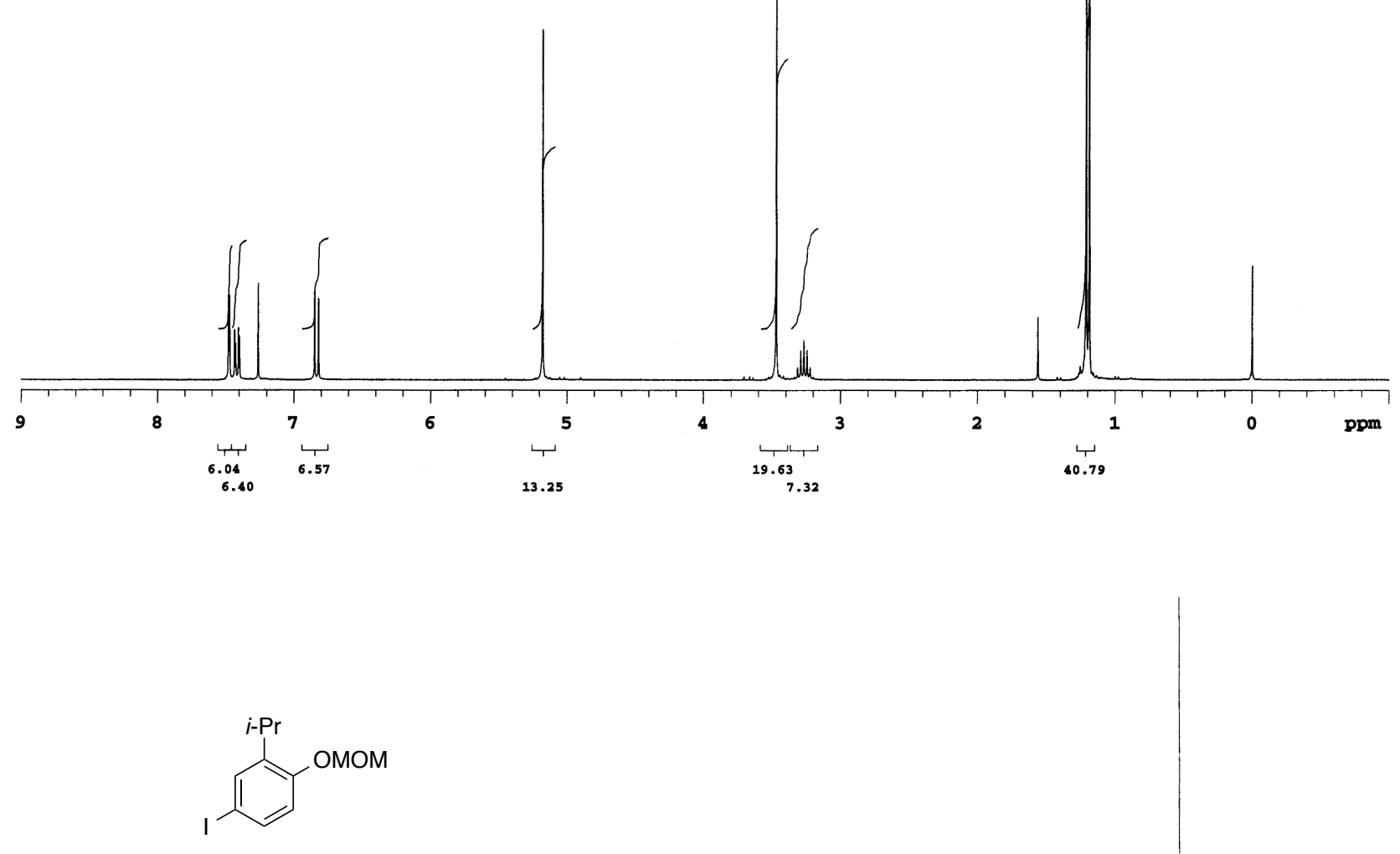

34

${ }^{13} \mathrm{C}$ NMR $\left(75 \mathrm{MHz}, \mathrm{CDCl}_{3}\right)$

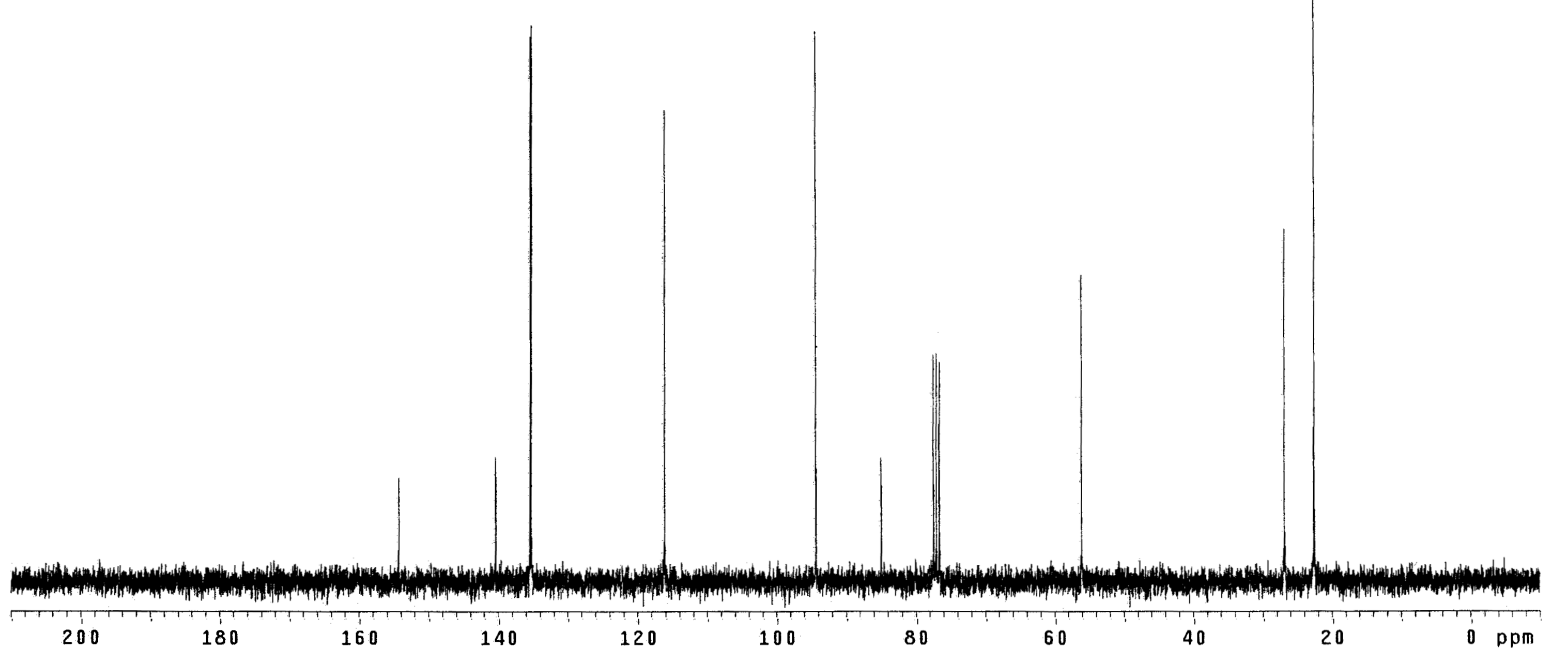




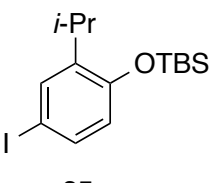

35

${ }^{1} \mathrm{H}$ NMR $\left(300 \mathrm{MHz}, \mathrm{CDCl}_{3}\right)$

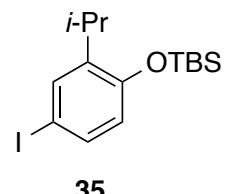

35

${ }^{13} \mathrm{C}$ NMR $\left(75 \mathrm{MHz}, \mathrm{CDCl}_{3}\right)$

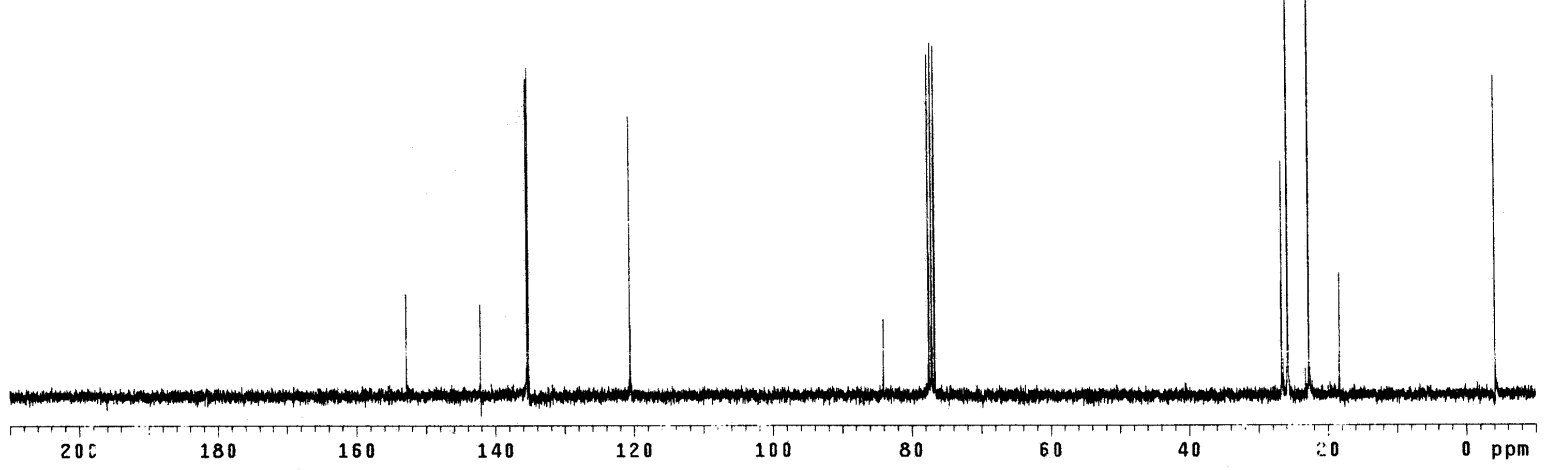

